DAMTP/92-31

\title{
Quantum Field Theories on Manifolds with Curved Boundaries: Scalar Fields
}

\author{
D.M. McAvity and H. Osborn \\ Department of Applied Mathematics and Theoretical Physics, \\ Silver Street, Cambridge, UK
}

A framework allowing for perturbative calculations to be carried out for quantum field theories with arbitrary smoothly curved boundaries is described. It is based on an expansion of the Green function for second order differential operators valid in the neighbourhood of the boundary and which is obtained from a corresponding expansion of of the associated heat kernel derived earlier for arbitrary mixed Dirichlet and Neumann boundary conditions. The first few leading terms in the expansion are sufficient to calculate all additional divergences present in a perturbative loop expansion as a consequence of the presence of the boundary. The method is applied to a general renormalisable scalar field theory in four dimensions using dimensional regularisation to two loops and expanding about arbitrary background fields. Detailed results are also specialised to an $O(n)$ symmetric model with a single coupling constant. Extra boundary terms are introduced into the action which give rise to either Dirichlet or generalised Neumann boundary conditions for the quantum fields. For plane boundaries the resulting renormalisation group functions are in accord with earlier results but here the additional terms depending on the extrinsic curvature of the boundary are found. Various consistency relations are also checked and the implications of conformal invariance at the critical point where the $\beta$ function vanishes are also derived. For a general scalar field theory, where the fields $\phi$ attain specified values $\hat{\varphi}$ on the boundary, the local Scrödinger equation for the wave functional defined by the functional integral under deformations of the boundary is also verified to two loops. The perturbative expansion for the wave functional is defined by expansion around the solution of the classical field equations satisfying the required boundary values and the counterterms necessary to derive a finite hamiltonian operator, which includes a functional laplace operator on the fields $\hat{\varphi}$, are found to the order considered. Consistency of the local Schrödinger equation with the renormalisation group to all orders in perturbation theory is also discussed. 


\section{Introduction}

Although quantum field theory has an enormous literature, papers devoted to the properties of and perturbative calculations for quantum fields in the vicinity of a boundary are relatively sparse. Nevertheless considering quantum field theory on a manifold with boundary is not without interest for the following reasons:

(i) The Casimir energy $[1,2,3]$ is obviously an effect which depends on the presence of boundaries and exemplifies the crucial zero point energy of quantum fields. However explicit calculation for non trivial geometries and for interactions are difficult and the usual renormalisation framework requires extension.

(ii) In statistical physics there are additional critical indices associated with phase transitions for physical observables measured near a boundary [4]. These may be calculated in the framework of the $\varepsilon$ expansion of the same quantum field theory used to obtain the bulk exponents but in the presence of a boundary [5].

(iii) In bag models, which may possibly be regarded as an approximation to QCD with some phenomenological validity, it is necessary to consider quantum fields inside cavities with suitable boundary conditions [6]. Various perturbative calculations in bag models have been undertaken based on using a multiple scattering expansion for the propagator in the presence of a boundary [7].

(iv) Open strings correspond to conformal field theories with additional operators attached to the boundary of the two dimensional world sheet [8].

(v) Cardy has shown how additional information on the structure of two dimensional conformal field theory may be obtained by considering the usual machinery of operator product expansions with boundary operators [9].

(vi) An alternative formulation of quantum field theory to the conventional approach may be given in terms of a Schrödinger like representation with wave functionals $\Psi(\hat{\varphi})$ defined by the functional integral over quantum fields on a manifold $\mathcal{M}$ which attain a specified value $\hat{\varphi}$ on the boundary $\partial \mathcal{M}$. Such wave functionals obey formally a Schrödinger like equation where the time differentiation corresponds to deformations of the boundary $[10,11]$. However the second order functional derivatives in the Hamiltonian operator involve singularities which require careful discussion. The solutions of the functional Schrödinger equation provided by the functional integral over fields on manifolds with a boundary are essentially identical to formal constructions of wave functionals satisfying the Wheeler-DeWitt equation in quantum gravity [12] which have been extensively explored recently.

However, in the presence of a boundary the usual efficient calculational techniques based on momentum space representations of the propagators are no longer valid, although in the case of plane boundaries there are possible extensions [5]. Here we wish to discuss quantum field theory on curved space with an arbitrary smoothly curved boundary. For simplicity at this stage we restrict our attention to a general renormalisable scalar field theory on a Euclidean space with a positive definite metric in four dimensions using dimensional regularisation, developing further the treatment some time ago of Symanzik 
[10]. However, our methods should be feasible for more interesting field theories, such as $\sigma$ models in two dimensions which are relevant for strings.

In order to carry out calculations for the short distance behaviour of amplitudes for a quantum field theory in the neighbourhood of a boundary it is crucial to be able to determine besides the structure of the leading singularities at coincident points $x^{\prime} \rightarrow x$ of the Green function $G_{\Delta}\left(x, x^{\prime}\right)$ for the elliptic operator $\Delta$, defined when the action is expanded to quadratic order around some background, also the behaviour as $x, x^{\prime}$ approach the boundary. Recently we have developed [13] an alternative to the multiple scattering expansion based on an analysis of the heat kernel $\mathcal{G}_{\Delta}\left(x, x^{\prime} ; \tau\right)$ corresponding to $e^{-\tau \Delta}$ as $\tau \rightarrow 0$ in the neighbourhood of a boundary for either Dirichlet and Neumann boundary conditions. The results depend on an extension of the usual DeWitt ansatz [14] to include an effectively semi-classical expansion about geodesics which undergo reflection at the boundary. The details of this expansion are summarised in appendix A. Throughout we maintain manifest reparameterisation invariance with respect to the coordinates $\hat{x}^{i}$ for $\partial \mathcal{M}$ and for the purposes of calculation, in the neighbourhood of $\partial \mathcal{M}$, adopt the coordinates $x^{\mu}=\left(x^{i}, y\right)$ where $y$ is the geodesic distance from $x^{\mu} \in \mathcal{M}$ to $x^{i}=\hat{x}^{i} \in \partial \mathcal{M}$ defined by the geodesic tangent to the unit normal $n^{\mu}$ at $\hat{x}^{i}$. In these coordinates the metric $g_{\mu \nu}$ on $\mathcal{M}$ is given by

$$
d s^{2}=\gamma_{i j}(\mathbf{x}, y) d x^{i} d x^{j}+d y^{2}, \quad n^{\mu}=(\mathbf{0}, 1)
$$

where $\gamma_{i j}(\mathbf{x}, 0)=\hat{\gamma}_{i j}(\hat{\mathbf{x}})$ is thus the induced metric on $\partial M$.

We assume that the operator $\Delta$, acting on sections of some vector bundle over $\mathcal{M}$, is of the form

$$
\Delta=-D^{2}+X, \quad D^{2}=\frac{1}{\sqrt{g}} D_{\mu} g^{\mu \nu} \sqrt{g} D_{\nu}, D_{\mu}=\partial_{\mu}+A_{\mu},
$$

for $X, A_{\mu}$ matrix valued fields on $\mathcal{M} . A_{\mu}$ can be regarded as an external background gauge field but may also be taken to include any appropriate spin connection. Assuming also that $\Delta$ is a symmetric operator acting on vector fields $\xi(x)$ over $\mathcal{M}$ the general form of the boundary conditions considered here, which is sufficient for most field theoretic applications, involve $\xi$ and its normal derivative on $\partial \mathcal{M}$ in the form

$$
\left.\left(\mathcal{P} n^{\mu} D_{\mu}+\psi\right) \xi\right|_{\partial \mathcal{M}}=0,\left.\quad(1-\mathcal{P}) \xi\right|_{\partial \mathcal{M}}=0, \quad \mathcal{P}^{2}=\mathcal{P}, \quad \mathcal{P} \psi=\psi \mathcal{P}=\psi
$$

for $\mathcal{P}(\hat{\mathbf{x}})$ a projection operator and $\psi(\hat{\mathbf{x}})$ a matrix valued function on $\partial \mathcal{M}$. Clearly from (1.3) taking $\mathcal{P}=0$ corresponds to pure Dirichlet boundary conditions while $\mathcal{P}=1$ is the Neumann case. With these boundary conditions the Green function $G_{\Delta}$ is defined by

$$
\Delta_{x} G_{\Delta}\left(x, x^{\prime}\right)=\delta^{d}\left(x, x^{\prime}\right) \equiv \frac{1}{\sqrt{g}} \delta^{d}\left(x-x^{\prime}\right) .
$$

In the interior of $\mathcal{M}$ the DeWitt asymptotic expansion of the heat kernel $\mathcal{G}_{\Delta}$ determines a corresponding expansion of $G_{\Delta}$ which for discussing renormalisable field theories in four dimensions may be restricted to the form [15]

$$
G_{\Delta}=\sum_{n=0}^{2} G_{n} a_{n}^{\Delta}+\bar{G}_{\Delta}
$$


for $a_{n}^{\Delta}\left(x, x^{\prime}\right)$ the Seeley-DeWitt coefficients, nonsingular for $x \approx x^{\prime}$, and the singular part of $G_{\Delta}$ is given entirely in terms of $G_{n}\left(x, x^{\prime}\right)$ where

$$
\begin{aligned}
& G_{0}=\frac{\Gamma\left(\frac{1}{2} d-1\right)}{4 \pi^{\frac{1}{2} d}} \frac{\Delta^{\frac{1}{2}}}{(2 \sigma)^{\frac{1}{2} d-1}}, \\
& G_{1}=\frac{\Gamma\left(\frac{1}{2} d-2\right)}{16 \pi^{\frac{1}{2} d}} \frac{\Delta^{\frac{1}{2}}}{(2 \sigma)^{\frac{1}{2} d-2}}+\frac{\mu^{-\varepsilon}}{\varepsilon} \frac{\Delta^{\frac{1}{2}}}{8 \pi^{2}}, \\
& G_{2}=\frac{\Gamma\left(\frac{1}{2} d-3\right)}{64 \pi^{\frac{1}{2} d}} \frac{\Delta^{\frac{1}{2}}}{(2 \sigma)^{\frac{1}{2} d-3}}-\frac{\mu^{-\varepsilon}}{\varepsilon} \frac{\Delta^{\frac{1}{2}}}{16 \pi^{2}} \sigma
\end{aligned}
$$

for $\sigma\left(x, x^{\prime}\right)$ the geodetic interval given by $\frac{1}{2} \int_{0}^{1} d s \dot{x}(s)^{2}$ for $x^{\mu}(s)$ the geodesic path from $x^{\mu}(0)=x^{\prime}$ to $x^{\mu}(1)=x . \Delta^{\frac{1}{2}}\left(x, x^{\prime}\right)$ is a nonsingular symmetric biscalar which may be absorbed in $a_{n}^{\Delta}$ but the expressions (1.6) are convenient for correspondence with previous results. The representation provided by $(1.5,6)$ is valid for an arbitrary dimension $d$ of $\mathcal{M}$, with $\partial \mathcal{M}$ of dimension $d-1$. However in the expressions for $G_{1}, G_{2}$ the pole in the first term of each on the r.h.s. of (1.6) at $\varepsilon=4-d \rightarrow 0$ has been subtracted so that there is a well defined limit for $d=4$, this ensures a convenient form for $\bar{G}_{\Delta}$ which is regular for $\varepsilon \rightarrow 0$ as well as $x \approx x^{\prime}$. The subtraction also represents the removal of infra-red divergences of the corresponding flat space Fourier transforms $k^{-4}, k^{-6}$ when $k \rightarrow 0$. In these pole terms an arbitrary mass scale $\mu$ has been introduced for dimensional consistency, as usual in dimensional regularisation. $G_{\Delta}$ is independent of $\mu$, any variation in $\mu$ may be compensated by a corresponding change in $\bar{G}_{\Delta}$ in (1.5).

In discussing a renormalisable field theory it is also sufficient to resort to just the first few terms in a covariant Taylor expansion of $a_{n}\left(x, x^{\prime}\right)$ about $x^{\prime}=x$,

$$
\begin{aligned}
a_{0}^{\Delta}= & I, \quad \Delta^{\frac{1}{2}} \approx 1+\frac{1}{12} R_{\mu \nu} \sigma^{\mu} \sigma^{\nu}-\frac{1}{24} \nabla_{\rho} R_{\mu \nu} \sigma^{\rho} \sigma^{\mu} \sigma^{\nu} \\
a_{1}^{\Delta} \approx & \left(\frac{1}{6} R-X\right) I-\left(\frac{1}{12} \partial_{\mu} R-\frac{1}{6} D^{\nu} F_{\mu \nu}-\frac{1}{2} D_{\mu} X\right) \sigma^{\mu} I \\
& +\left(-\frac{1}{6} D_{\mu} D_{\nu} X+\frac{1}{12} F_{\mu \sigma} F_{\nu}{ }^{\sigma}-\frac{1}{12} D_{\mu} D^{\sigma} F_{\nu \sigma}+\frac{1}{40} \nabla_{\mu} \nabla_{\nu} R+\frac{1}{120} \nabla^{2} R_{\mu \nu}\right. \\
& \left.\quad+\frac{1}{180}\left(R^{\sigma \rho} R_{\mu \sigma \nu \rho}+R_{\mu \sigma \rho \tau} R_{\nu}{ }^{\sigma \rho \tau}-2 R_{\mu \sigma} R_{\nu}{ }^{\sigma}\right)\right) \sigma^{\mu} \sigma^{\nu} I \\
a_{2}^{\Delta} \approx & \left(\frac{1}{2}\left(\frac{1}{6} R-X\right)^{2}-\frac{1}{6} D^{2} X+\frac{1}{12} F_{\mu \nu} F^{\mu \nu}\right. \\
& \left.+\frac{1}{180}\left(R_{\mu \nu \sigma \rho} R^{\mu \nu \sigma \rho}-R_{\mu \nu} R^{\mu \nu}\right)+\frac{1}{30} \nabla^{2} R\right) I,
\end{aligned}
$$

where $\sigma^{\mu}\left(x, x^{\prime}\right)=g^{\mu \nu}(x) \partial_{\nu} \sigma\left(x, x^{\prime}\right)$ and $I\left(x, x^{\prime}\right)$ is the matrix giving parallel transport along the geodesic from $x^{\prime}$ to $x$ defined by $\sigma^{\mu} D_{\mu} I=0, I(x, x)=1 . F_{\mu \nu}$ is the usual field strength formed from $A_{\mu}$. With the decomposition of $G_{\Delta}$ given in (1.5) extensive calculations at two and more loops have been undertaken using formulae for the singular parts of the products of $G_{n}$ such as

$$
G_{0}^{2} \sim \frac{\mu^{-\varepsilon}}{8 \pi^{2} \varepsilon} \delta^{d}, \quad G_{0}^{3} \sim \frac{\mu^{-2 \varepsilon}}{\left(16 \pi^{2}\right)^{2}} \frac{1}{\varepsilon} \frac{1}{2}\left(\nabla^{2}+\frac{1}{6} R\right) \delta^{d}, \quad G_{0}^{2} G_{1} \sim \frac{\mu^{-2 \varepsilon}}{\left(16 \pi^{2}\right)^{2}}\left(\frac{2}{\varepsilon^{2}}+\frac{1}{\varepsilon}\right) \delta^{d}
$$

Similar relations involving derivatives may be found in ref. (15), in general at $\ell$ loops dimensional consistency requires a factor $\mu^{-\ell \varepsilon}$. 
In the neighbourhood of $\partial \mathcal{M}$ the expansion (1.6) is no longer sufficient for revealing all singular contributions in amplitudes at one or more loops. If $\hat{\sigma}\left(\hat{\mathbf{x}}, \hat{\mathbf{x}}^{\prime}\right)$ is the geodetic interval on $\partial \mathcal{M}$, as determined by the metric $\hat{\gamma}_{i j}(\hat{\mathbf{x}})$, then, for coordinates corresponding to the metric (1.1), assuming

$$
\hat{\sigma}^{i}, y, y^{\prime}=\mathrm{O}(\epsilon), \quad \hat{\sigma}=\frac{1}{2} \hat{\gamma}_{i j} \hat{\sigma}^{i} \hat{\sigma}^{j}=\mathrm{O}\left(\epsilon^{2}\right)
$$

and with the choice of gauge

$$
A_{\mu}=(\mathbf{A}, 0)
$$

$G_{\Delta}$ may now expanded as

$$
G_{\Delta}=\sum_{n=0}^{3}\left(G_{n}^{B}+\bar{G}_{n}^{B}\right)+\bar{G}_{\Delta}^{B}, \quad G_{n}^{B}, \bar{G}_{n}^{B}=\mathrm{O}\left(\epsilon^{n+2-d}\right)
$$

with $\bar{G}_{\Delta}^{B}$ regular both for $x^{\prime} \rightarrow x$ and also on $\partial \mathcal{M}$ for $d=4$. $G_{n}^{B}$ correspond to the singular part of $G_{\Delta}$ as $x^{\prime} \rightarrow x$ while $\bar{G}_{n}^{B}$, which may be determined from the heat kernel expansion described in appendix $\mathrm{A}$, are non singular when $x^{\prime}=x$ but are necessary in order to satisfy the boundary conditions and lead to additional divergences as the boundary is approached. If we define

$$
\begin{aligned}
G_{r}(u)= & \frac{\Gamma\left(\frac{1}{2} d-r\right)}{4 \pi^{\frac{1}{2} d}}\left(u^{2}+2 \hat{\sigma}\right)^{r-\frac{1}{2} d}, \quad G_{r, s}(u)=\int_{0}^{\infty} d z z^{s} G_{r}(z+u), \\
& G_{r, s+1}(u)+u G_{r, s}(u)=\frac{1}{2} s G_{r+1, s-1}(u)+\frac{1}{2} \delta_{s 0} G_{r+1}(u),
\end{aligned}
$$

then explicitly for $n=0,1$ we may obtain for general $\Delta$ and boundary conditions as in $(1.2,3)$

$$
\begin{aligned}
G_{0}^{B}= & G_{1}(v) \hat{I}, \quad G_{1}^{B}=G_{0}(v) u K_{i j} \hat{\sigma}^{i} \hat{\sigma}^{j} \hat{I}, \quad v=y-y^{\prime} \\
\bar{G}_{0}^{B}= & G_{1}(u) \mathcal{P}_{-} \hat{I}, \quad \mathcal{P}_{-}=2 \mathcal{P}-1, \quad u=y+y^{\prime} \\
\bar{G}_{1}^{B}= & G_{1,0}(u) 2 \psi \hat{I}+G_{0}(u) u K_{i j} \hat{\sigma}^{i} \hat{\sigma}^{j} \mathcal{P}_{-} \hat{I} \\
& +2 y y^{\prime}\left(G_{0,0}(u) K-2 G_{-1,0}(u) K_{i j} \hat{\sigma}^{i} \hat{\sigma}^{j}\right) \mathcal{P}_{-} \hat{I} \\
& +\left(G_{1,0}(u)-G_{0,2}(u)\right) K \mathcal{P} \hat{I}+2 G_{-1,2}(u) K_{i j} \hat{\sigma}^{i} \hat{\sigma}^{j} \mathcal{P} \hat{I} \\
& -G_{1}(u) 2 \mathcal{P} \hat{\sigma}^{i} \hat{D}_{i} \mathcal{P} \hat{I}+G_{0,0}(u)\left(4 y^{\prime} \mathcal{P} \hat{\sigma}^{i} \hat{D}_{i} \mathcal{P}-4 y \hat{\sigma}^{i} \hat{D}_{i} \mathcal{P} \mathcal{P}\right) \hat{I}
\end{aligned}
$$

where the coefficients, $\mathcal{P}, \psi, K_{i j}$, are evaluated at $\hat{x} . \hat{I}\left(\hat{\mathbf{x}}, \hat{\mathbf{x}}^{\prime}\right)$, defined similarly to $I\left(x, x^{\prime}\right)$ earlier, is the matrix corresponding to parallel transport along the geodesic in $\partial \mathcal{M}$ from $\hat{\mathbf{x}}^{\prime}$ to $\hat{\mathbf{x}}$ for the connection $\hat{\mathbf{A}}=\left.\mathbf{A}\right|_{y=0}$ and $\hat{D}_{i}$ denotes the corresponding covariant derivative on the boundary, $\hat{D}_{i} \mathcal{P}=\partial_{i} \mathcal{P}+\left[\hat{A}_{i}, \mathcal{P}\right] . K_{i j}$ is here the extrinsic curvature of the boundary, with coordinates given by $(1.1) K_{i j}=-\left.\frac{1}{2} \partial_{y} \gamma_{i j}\right|_{y=0}, \quad K=\hat{\gamma}^{i j} K_{i j}$. Clearly the presence of a curved boundary, so that $K_{i j}$ is non zero, is a significant complication. For $\hat{\sigma}=0$ the integrals in the definition of $G_{r, s}$ in (1.12) are easily evaluated but in general the form given is sufficient for further calculations. To obtain (1.13) the terms $\sum_{n} G_{n}^{B}$ in (1.11) 
may be derived by expanding the singular contributions in (1.5) using (1.6) and (1.7) and the remaining terms $\sum_{n} \bar{G}_{n}^{B}$ can then be verified to be in accord with the essential Green function equation (1.4) and the boundary conditions by expanding about $y=0$, $\Delta=\Delta_{0}+\Delta_{1}+\ldots, \Delta_{n}=\mathrm{O}\left(\epsilon^{n-2}\right)$, regarding $\partial_{y}, \hat{D}_{i}$ as $\mathrm{O}\left(\epsilon^{-1}\right)$ in addition to $y=\mathrm{O}(\epsilon)$ as in (1.9), and using results such as

$$
\begin{aligned}
\partial_{i} G_{r}(u) & =-2 G_{r-1}(u) \hat{\sigma}_{i}, \quad \partial_{y} G_{r}(u)=-2 G_{r-1}(u) u, \\
\partial_{y} G_{r, s}(u) & =-s G_{r, s-1}(u)+\delta_{s 0} G_{r}(u), \\
\Delta_{0} G_{r}(u) & =2(2(r-1)+f) G_{r-1}(u), \quad f=\hat{\nabla}^{2} \hat{\sigma}-d+1,
\end{aligned}
$$

where $\Delta_{0}=-\hat{D}^{2}-\partial_{y}^{2}$ and $\hat{\nabla}_{i} \hat{\sigma}^{j}=\delta_{i}^{j}-\frac{1}{3} \hat{R}_{k i \ell} \hat{\sigma}^{k} \hat{\sigma}^{\ell}+\ldots$, for $\hat{R}_{i j k \ell}$ the Riemann tensor formed from the induced metric on $\partial \mathcal{M}$. The complete expression for the $n=2$ terms in (1.11) may be found from the results obtained elsewhere for the heat kernel to this order, in appendix A, in (A.10) we give the results for $n=2$ involving $X$ and $\psi$. These involve functions such as $G_{2}(u)$ where it is necessary to subtract a pole term at $\varepsilon=0$ as in (1.6).

Instead of the result given by (1.13) an alternate equivalent form for $\bar{G}_{1}^{B}$ is given by

$$
\begin{aligned}
\bar{G}_{1}^{B}= & G_{0}(u) u\left(1+\frac{y y^{\prime}}{\hat{\sigma}}\right) K_{i j} \hat{\sigma}^{i} \hat{\sigma}^{j} \mathcal{P}_{-} \hat{I} \\
& +\left(2 y y^{\prime} G_{0,0}(u) \mathcal{P}_{-}+\frac{1}{d-1} G_{1,0}(u) \mathcal{P}-G_{0,2}(u) \mathcal{P}\right)\left(K-(d-1) \frac{K_{i j} \hat{\sigma}^{i} \hat{\sigma}^{j}}{2 \hat{\sigma}}\right) \hat{I} \\
& +G_{1,0}(u)\left(2 \psi+\frac{d-2}{d-1} K \mathcal{P}\right) \hat{I} \\
& -G_{1}(u) 2 \mathcal{P} \hat{\sigma}^{i} \hat{D}_{i} \mathcal{P} \hat{I}+G_{0,0}(u)\left(4 y^{\prime} \mathcal{P} \hat{\sigma}^{i} \hat{D}_{i} \mathcal{P}-4 y \hat{\sigma}^{i} \hat{D}_{i} \mathcal{P} \mathcal{P}\right) \hat{I}
\end{aligned}
$$

This expression is in accord with the result that for a sphere of radius $a$ when $K_{i j}=\hat{\gamma}_{i j} / a$ and also in the Neumann case for $\psi=-\frac{1}{2}(d-2) / a$ it is possible to find the Green function for $-\partial^{2}$ in terms of the elementary flat space result for no boundary by the method of images. In this particular case (1.15) is independent of the special functions $G_{r, s}$.

In any amplitude with superficial degree of divergence $D$ then expanding $G_{\Delta}$ associated with any internal line reduces $D$ to $D-2 n$ for the contribution of the $G_{n} a_{n}^{\Delta}$ term in (1.5) and to $D-n$ for the terms $G_{n}^{B}, \bar{G}_{n}^{B}$ in (1.11). For vacuum diagrams for renormalisable theories in four dimensions $D=4$ while for divergences associated with the boundary $D=3$.

At one loop amplitudes are determined by the functional determinant of $\Delta$ and standard methods give

$$
-\ln \operatorname{det} \Delta=\int_{0}^{\infty} \frac{d \tau}{\tau} \operatorname{Tr}\left(e^{-\tau \Delta}\right),
$$

and the singular part for $d \rightarrow 4$ is then, using previous results $[13,16]$ for the asymptotic form of $\operatorname{Tr}\left(e^{-\tau \Delta}\right)$ as $\tau \rightarrow 0$,

$$
-\ln \operatorname{det} \Delta \sim \frac{2}{\varepsilon} \frac{\mu^{-\varepsilon}}{16 \pi^{2}}\left\{\int_{\mathcal{M}} d v \operatorname{tr}\left(\frac{1}{2}\left(\frac{1}{6} R-X\right)^{2}+\frac{1}{12} F_{\mu \nu} F^{\mu \nu}+\frac{1}{180}\left(R_{\mu \nu \sigma \rho} R^{\mu \nu \sigma \rho}-R_{\mu \nu} R^{\mu \nu}\right)\right)\right.
$$




$$
\begin{aligned}
& +\int_{\partial \mathcal{M}} d S \operatorname{tr}\left(\frac{1}{2} \mathcal{P}_{-}\left(\frac{1}{6} \partial_{n} R-\partial_{n} X\right)+\left(2 \psi+\frac{1}{3} K\right)\left(\frac{1}{6} \hat{R}-X\right)\right. \\
& \quad+\frac{2}{3} \psi R_{n n}+\frac{7}{15} \psi K_{i j} K^{i j}+\frac{1}{15} \psi K^{2}+\frac{4}{3} \psi^{2}(K+\psi) \\
& \quad+\frac{1}{10} K R_{n n}+\frac{1}{30} K^{i j} R_{i n j n}-\frac{1}{90} K^{i j} \hat{R}_{i j}+\frac{1}{3} \mathcal{P}_{-} \hat{D}_{i} \mathcal{P} F^{i}{ }_{n} \\
& \quad+\frac{1}{63}\left(\left(2 \mathcal{P}-\frac{19}{6}\right) K^{3}+\left(\frac{18}{5} \mathcal{P}+2\right) K K_{i j} K^{i j}+\left(\frac{59}{30}-\frac{4}{5} \mathcal{P}\right) K_{i j} K^{j k} K_{k}{ }^{i}\right) \\
& \left.\left.\quad-\frac{7}{15} \hat{D}_{i} \mathcal{P} \hat{D}^{i} \mathcal{P} K+\frac{1}{15} \hat{D}_{i} \mathcal{P} \hat{D}_{j} \mathcal{P} K^{i j}-\frac{4}{3} \hat{D}_{i} \mathcal{P} \hat{D}^{i} \mathcal{P} \psi\right)\right\}
\end{aligned}
$$

with $d v=d^{d} x \sqrt{g}$ and $d S=d^{d-1} \hat{\mathbf{x}} \sqrt{\hat{\gamma}}$.

Beyond one loop calculations become more involved. Using the representation outlined above for the singular part of $G_{\Delta}$ in the neighbourhood of a boundary we have undertaken calculations at two loops for the simplest renormalisable field theory in four dimensions, i.e. purely scalar field theory with a multi-component scalar field $\phi_{i}$ represented by an action

$$
S(\phi, g)=\int_{\mathcal{M}} d v\left(\frac{1}{2} \partial_{\mu} \phi_{i} \partial^{\mu} \phi_{i}+V(\phi)\right), \quad V(\phi)=\frac{1}{24} g_{i j k \ell} \phi_{i} \phi_{j} \phi_{k} \phi_{\ell}+\mathrm{O}\left(\phi^{3}\right)
$$

with $g$ denoting generically the complete set of couplings, including mass terms, on which $V$ depends, for $v_{I}(\phi)$ a basis of polynomials of degree 4 we may write $V(\phi)=\sum_{I} g_{I} v_{I}(\phi)$. In order to take account the effects of the boundary $\partial \mathcal{M}$ we also add to $S$ an extra surface term $\hat{S}(\phi, \hat{g})$ which is a integral over the boundary of a local scalar formed from $\phi$ and its normal derivative $\partial_{n} \phi$ and which depends on additional couplings, or sources, $\hat{g}$. We also follow the usual background field technique expanding

$$
\phi=\varphi+f,
$$

for some fixed background field $\varphi$ and assume boundary conditions on $\varphi$ so that $S+\hat{S}$ has no terms linear in $f$ when $\varphi$ satisfies the classical equation of motion $-\nabla^{2} \varphi+V^{\prime}(\varphi)=0$. The same boundary conditions are then imposed on $\phi$, which therefore entails corresponding boundary conditions for the fluctuations $f$. The background field method allows an efficient procedure for calculating divergent parts of vacuum self energy diagrams as local dimension 4 functions of $\varphi$ and hence, since $\phi$ and $\varphi$ satisfy the same boundary conditions, obtaining the necessary counterterms $S_{\text {c.t. }}(\phi, g), \hat{S}_{\text {c.t. }}(\phi, g, \hat{g})$ to ensure that the functional integral

$$
\int d[\phi] e^{-S_{0}(\phi, g, \hat{g})}, \quad S_{0}=\mu^{-\varepsilon}\left(S+S_{\text {c.t. }}+\hat{S}+\hat{S}_{\text {c.t. }}\right)
$$

defines a finite measure over fields $\phi$ satisfying the required boundary conditions. The factor $\mu^{-\varepsilon}$ in $S_{0}$ ensures that the couplings $g, \hat{g}$, as well as the fields $\phi$, retain their canonical dimensions although the quantum field theory is formally extended to $d$ dimensions.

In the next section we apply these methods to the situation where $\hat{S}$ is chosen so that $\left.\phi\right|_{\partial \mathcal{M}}=\hat{\varphi}$ for $\hat{\varphi}_{i}(\hat{\mathbf{x}})$ an arbitrary smooth boundary field. This corresponds to requiring that 
the quantum field $f$ in (1.19) satisfies Dirichlet boundary conditions on $\partial \mathcal{M}$. In this case it is essential, besides the usual counterterms on $\mathcal{M}$ necessary for finiteness, to introduce additional local counterterms on $\partial \mathcal{M}$ linear in $\partial_{n} \phi$ and also in $K$ which are constrained by power counting to have overall dimension 3. These are calculated to two loops making use of the expansion (1.11) for the Green function $G_{\Delta}$. The renormalisation group equations which now include terms involving the boundary operator $\partial_{n} \phi$ are also discussed. Similar calculations are also undertaken in section 3 for the case when $\hat{S}$ is chosen such that $\partial_{n} \phi$ is related to $\phi$ on $\partial \mathcal{M}$ and $f$ satisfies generalised Neumann boundary conditions. In both cases the final results are also specialised to the $O(n)$ symmetric case, where there is a single coupling $g$ and some results were also obtained by Diehl and Diettrich [5]. As usual with dimensional regularisation the renormalisation group equations determine the higher order poles in $\varepsilon$ of the counterterms in terms of the simple poles and these conditions are verified by our two loop results. The details of the calculation, for either the Dirichlet or Neumann case, are relegated to appendix B. They depend on the detailed form of the expansion given by (1.11), (1.12) and (1.13) or (1.15). A brief summary of the important results for the heat kernel for a general second order operator $\Delta$ of the form (1.2) with boundary conditions (1.3) in our treatment is given in appendix A. In section 4 consistency conditions are derived under local Weyl rescalings of the metric which in effect determine the $K$ dependent counterterms in terms of those necessary, on flat space and for a plane boundary, to define local composite operators on $\partial \mathcal{M}$. These results allow the derivation of a local renormalisation group equation which is discussed for the $O(n)$ symmetric field theory at the critical point where $\hat{\beta}(g)=0$.

In section 5 we return to the situation for a general scalar field theory where the quantum field $\phi$ attains a fixed boundary value $\hat{\varphi}$ on $\partial \mathcal{M}$ and the functional integral (1.20) defines a wave functional $\hat{\Psi}(\hat{\varphi})$. Following earlier work by Symanzik [10], and in two dimensions by Lüscher et al [11], this is shown, to two loops in a semiclassical expansion with the background field $\varphi$ in (1.19) solving the classical equations of motion with the boundary condition $\varphi=\hat{\varphi}$ on $\partial \mathcal{M}$, to satisfy a local Schrödinger equation of the form

$$
\left(\hbar \frac{\delta}{\delta t(\hat{\mathbf{x}})}+\mathcal{H}_{0}(\hat{\mathbf{x}})\right) \hat{\Psi}(\hat{\varphi})=0
$$

$\delta t(\hat{\mathbf{x}})$ represents an arbitrary local deformation of the boundary $\partial \mathcal{M}$ along the normal $n^{\mu}(\hat{\mathbf{x}})$ and $\mathcal{H}_{0}(\hat{\mathbf{x}})$ is a local Hamiltonian operator. To leading order in the semiclassical expansion $\mathcal{H}_{0} \rightarrow \mathcal{H}$ where the functional differential operator is

$$
\mathcal{H}=-\frac{1}{2} \frac{\delta^{2}}{\delta \hat{\varphi}^{2}}+V(\hat{\varphi})+\frac{1}{2} \hat{\gamma}^{p q} \partial_{p} \hat{\varphi}_{i} \partial_{q} \hat{\varphi}_{i}
$$

although $\mathcal{H}_{0}$ contains counterterms reflecting those occurring in $S_{0}$ and also since the second functional derivative $\delta^{2} / \delta \hat{\varphi}(\hat{\mathbf{x}}) \delta \hat{\varphi}\left(\hat{\mathbf{x}}^{\prime}\right)$ is ill defined in general as $\hat{\mathbf{x}}^{\prime} \rightarrow \hat{\mathbf{x}}$. Such divergences are demonstrated here to be fully resolved by use of dimensional regularisation. Furthermore the structure of $\mathcal{H}_{0}$ is shown to be determined in terms of $S_{0}$ to all orders by use of the renormalisation group. Some mathematical details necessary to verify (1.21) are contained in appendix C. Finally in section 6 a few concluding remarks are made. 


\section{Quantum Scalar Field Theory with Prescribed Boundary Values}

In this section we consider the quantum field theory defined by the action (1.18) with the additional surface contribution

$$
\hat{S}(\phi, \hat{\varphi})=\int_{\partial \mathcal{M}} d S(\phi-\hat{\varphi})_{i} \partial_{n} \phi_{i},
$$

where $\hat{\varphi}(\hat{\mathbf{x}})$ may be regarded as a source defining the surface operator $\partial_{n} \phi(\hat{\mathbf{x}})=\left.\partial_{y} \phi(x)\right|_{y=0}$.

When $\phi$ is expanded about a background $\varphi$ as in (1.19) we may write $S+\hat{S}=$ $S_{\mathrm{c}}+S_{1}+S_{2}+\ldots$ with $S_{n}=\mathrm{O}\left(f^{n}\right)$ and $S_{\mathrm{c}}=S(\varphi, g)+\hat{S}(\varphi, \hat{\varphi})$. It is then straightforward to see that

$$
S_{1}=\int_{\mathcal{M}} d v f_{i}\left(-\nabla^{2} \varphi_{i}+V_{i}^{\prime}(\varphi)\right)+\int_{\partial \mathcal{M}} d S \partial_{n} f_{i}(\varphi-\hat{\varphi})_{i},
$$

so that the appropriate boundary conditions to impose in a perturbative expansion are

$$
\left.\phi\right|_{\partial \mathcal{M}}=\hat{\varphi},\left.\quad f\right|_{\partial \mathcal{M}}=0 .
$$

Also with this boundary condition

$$
S_{2}=\frac{1}{2} \int_{\mathcal{M}} d v f_{i}(\Delta f)_{i}, \quad \Delta=-\nabla^{2}+V^{\prime \prime}(\varphi),
$$

so that, acting on fields $f$ satisfying (2.4), $\Delta$ is a symmetric operator of the necessary form (1.2), with $X=V^{\prime \prime}(\varphi)$, and the associated Green function $G_{i j}\left(x, x^{\prime}\right)$, which corresponds to the propagator for internal lines in a perturbative expansion, is therefore defined by requiring Dirichlet boundary conditions. The higher order terms in the expansion in $f$ as usual generate the vertices for Feynman diagrams in a perturbative expansion, in this theory they arise just from $S_{3}$, depending on $V^{\prime \prime \prime}(\varphi)$, and $S_{4}$ with no interactions restricted just to $\partial \mathcal{M}$.

For considering correlation functions of $\phi$ in this quantum field theory with boundary conditions (2.3) the essential functional integral is

$$
e^{W(\hat{\varphi} ; J)}=\int_{\left.\phi\right|_{\partial \mathcal{M}}=\hat{\varphi}} d[\phi] e^{-S_{0}(\phi)+\int_{\mathcal{M}} d v J_{i} \phi_{i}} .
$$

The counterterms in $S_{0}$ are chosen so that $W(\hat{\varphi} ; J)$ is finite for arbitrary external sources $J_{i}(x)$. For calculation it is convenient to define

$$
\Gamma(\hat{\varphi} ; \varphi)=W(\hat{\varphi} ; J)-\int_{\mathcal{M}} d v J_{i} \varphi_{i},
$$

where $\varphi$ is assumed to be an arbitrary smooth field on $\mathcal{M}$, with boundary value $\hat{\varphi}$, while $J$ is constrained to be defined in terms of $\varphi$, with an invertible relationship $J \leftrightarrow \varphi$. For a quantum field theory without any boundary it is conventional to choose $J$ so that $\langle\phi\rangle=\varphi$, to all orders in the loop expansion, and then $\Gamma(\varphi)$ is the generating functional of connected 
one particle irreducible amplitudes. This contains the only primitive divergences requiring renormalisation by appropriate counterterms. In a perturbative expansion $\Gamma$ may be calculated via the background field method, taking $\phi=\varphi+f$ with $d[\phi]=d[f]$, when to ensure $\langle f\rangle=0$ it is necessary to require $J=J_{\mathrm{c}}+\ldots$ for $J_{\mathrm{c}}(\varphi) \equiv \mu^{-\varepsilon}\left(-\nabla^{2} \varphi+V^{\prime}(\varphi)\right)$. However, in the presence of a boundary this condition on $\langle f\rangle$ necessitates that $J(x)$ is singular as $x$ approaches the boundary, assuming the background $\varphi$ is smooth in the neighbourhood of $\partial \mathcal{M}$. This is a consequence of the fact that one particle reducible amplitudes also have primitive divergences, in the presence of a boundary, requiring corresponding contributions to $\hat{S}_{\text {c.t. }}$. For the purpose of the calculations of the counterterms required by the presence of a boundary undertaken here, it is simpler to choose $J=J_{\mathrm{c}}$, without any higher order corrections, to define $\Gamma(\hat{\varphi} ; \varphi)$ in $(2.5)$ and (2.6). This choice, together with the boundary condition on $\varphi$, cancels the linear term $S_{1}$ given by (2.2) in the expansion of the classical action although in this case it is necessary to include one particle irreducible graphs in the perturbative expansion beyond one loop. Since with dimensional regularisation there is a unique separation $\langle\varphi\rangle=\langle\varphi\rangle_{\text {n.s. }}+\langle\varphi\rangle_{\text {s. }}$, where $\langle\varphi\rangle_{\text {n.s. }},\langle\varphi\rangle_{\text {s. }}$ are respectively non-singular, singular as the boundary is approached, an alternative possibility would be,to require $\langle\varphi\rangle_{\text {n.s. }}=0$ but this is not pursued here. In this framework it straightforward to define a Scrödinger wave functional $\hat{\Psi}(\hat{\varphi})$ by $\hat{\Psi}(\hat{\varphi})=\exp W(\hat{\varphi} ; 0)=\exp \Gamma\left(\hat{\varphi} ; \varphi_{\mathrm{c}}\right)$ where $\varphi_{\mathrm{c}}$ is the classical solution to $-\nabla^{2} \varphi_{\mathrm{c}}+V^{\prime}\left(\varphi_{\mathrm{c}}\right)=0$ prescribed by the boundary value $\hat{\varphi}$.

Following standard procedure the structure of possible counterterms is determined by power counting. As usual

$$
S_{\text {c.t. }}(\phi, g)=\int_{\mathcal{M}} d v\left(\frac{1}{2} \partial_{\mu} \phi_{i} A_{i j} \partial^{\mu} \phi_{j}+V_{\text {c.t. }}(\phi)+C(\lambda)\right),
$$

with $V_{\text {c.t. }}(\phi)$ a polynomial of degree 4 in $\phi$ which is also taken to include terms linear in $R$ which are quadratic in $\phi . C(\lambda)$ contains all additional counterterms which involve no operators, or dependence on $\phi$, but are necessary for a curved space background, it may be written as

$$
\begin{aligned}
C(\lambda)= & -\left(a F+b G+c R^{2}\right), \quad \lambda=(a, b, c), \\
& F=R^{\mu \nu \rho \sigma} R_{\mu \nu \rho \sigma}-\frac{4}{d-2} R^{\mu \nu} R_{\mu \nu}+\frac{2}{(d-2)(d-1)} R^{2}, \\
& G=R^{\mu \nu \rho \sigma} R_{\mu \nu \rho \sigma}-4 R^{\mu \nu} R_{\mu \nu}+R^{2},
\end{aligned}
$$

where $\lambda$ is determined perturbatively in terms of the dimensional coupling $g$. The additional counterterms restricted to the boundary are of dimension 3 and have the general form

$$
\hat{S}_{\text {c.t. }}(\phi, g, \hat{\varphi})=\int_{\partial \mathcal{M}} d S\left(-\hat{\varphi}_{\text {c.t. } i}(\hat{\varphi}) \partial_{n} \phi_{i}+\rho(\hat{\varphi}) K+\hat{C}(\hat{\lambda})\right),
$$

using $\left.\phi\right|_{\partial \mathcal{M}}=\hat{\varphi}$ and also that with dimensional regularisation there are no linearly divergent terms which are $\mathrm{O}\left(\hat{\varphi}^{2}\right)$. Assuming minimal subtraction $S_{\text {c.t. }}$ and $\hat{S}_{\text {c.t. }}$ contain only poles in $\varepsilon$. $\hat{C}$, with $\hat{\lambda}$ denoting the coefficients in some basis, includes all terms which are proportional to any dimension 3 scalar, independent of $\hat{\varphi}$, formed solely from the extrinsic 
and intrinsic curvature on $\partial \mathcal{M}$, such as appear in the surface terms in (1.17) not involving $\psi$ and $X$.

Due to the complexity of the calculations necessary to determine such contributions we do not consider them here but focus primarily on $\hat{\varphi}_{\text {c.t. }}$, which has in effect been calculated previously to two loop order by Diehl and Dietrich, and also on $\rho$. Of course $S_{\text {c.t. }}$ in the form (2.7) has been calculated extensively before at two and higher loops although it is necessary to redetermine all contributions to $A$ since any integration by parts of derivatives may lead to surface terms. It is also necessary to determine any divergences proportional to total derivatives which are usually discarded. Using the background field method $S_{\text {c.t. }}$ may be calculated with $\partial_{n} \phi \rightarrow \partial_{n} \varphi$. It is easy to see that $\hat{\varphi}_{\text {c.t. }}(\hat{\varphi})$ is linear in $\hat{\varphi}$ and depends in general on $V^{\prime \prime \prime}(\hat{\varphi})$ while $\rho(\hat{\varphi})$ is quadratic in $\hat{\varphi}$ and is proportional to $V^{\prime \prime}(\hat{\varphi})$ or $V^{\prime \prime \prime}(\hat{\varphi})^{2}$ and no other $\hat{\varphi}$ dependent terms are required in $(2.8)$.

In general for a perturbative expansion of the functional integral (2.5) with a background field $\varphi$

$$
\Gamma(\hat{\varphi} ; \varphi)=-S_{0}(\varphi)+\sum_{\ell=1} \Gamma^{(\ell)}(\hat{\varphi} ; \varphi)
$$

where $\Gamma^{(\ell)}(\varphi)$ is the connected amplitude at $\ell$ loops and $S_{0}(\varphi)$ contains the necessary counterterms to ensure that $\Gamma(\hat{\varphi} ; \varphi)$ is finite. At one loop

$$
\Gamma^{(1)}=-\frac{1}{2} \ln \operatorname{det} \Delta,
$$

and using (1.17) it is easy to see that to ensure cancellation of the poles in $\varepsilon$ it is sufficient to take in $(2.8)$

$$
\hat{\varphi}_{\text {c.t. } i}^{(1)}(\hat{\varphi})=-\frac{1}{16 \pi^{2} \varepsilon} \frac{1}{2} V_{i j j}^{\prime \prime \prime}(\hat{\varphi}), \quad \rho^{(1)}(\hat{\varphi})=-\frac{1}{16 \pi^{2} \varepsilon} \frac{1}{3} V_{j j}^{\prime \prime}(\hat{\varphi}) .
$$

Beyond one loop the calculational details are non trivial and are mostly relegated to appendix B. The essential two loop vacuum diagrams are shown in fig.1 and accordingly we decompose $\Gamma^{(2)}=\Gamma_{a}+\Gamma_{b}+\ldots$. Of course at two loops there are one loop subdivergences which are removed by one loop counterterms. For the scalar field theory defined by (1.18) and (2.1) these are given solely by

$$
V_{\text {c.t. }}^{(1)}-c^{(1)} R^{2}=\frac{1}{16 \pi^{2} \varepsilon} \frac{1}{2} \tilde{V}_{i j} \tilde{V}_{i j}, \quad \tilde{V}_{i j}(\phi)=V_{i j}^{\prime \prime}(\phi)-\frac{1}{6} R \delta_{i j} .
$$

The amplitude for fig.1a, with appropriate counterterms, is then

$$
\begin{aligned}
\Gamma_{a} & =-\frac{\mu^{\varepsilon}}{8} \int d v g_{i j k \ell} G_{i j}\left|G_{k \ell}\right|-\frac{1}{16 \pi^{2} \varepsilon} \frac{1}{2} \int d v g_{i j k \ell} \tilde{V}_{i j}(\varphi) G_{k \ell} \mid \\
& =\frac{1}{8} \int d v g_{i j k \ell}\left(\frac{4 \mu^{-\varepsilon}}{\left(16 \pi^{2} \varepsilon\right)^{2}} \tilde{V}_{i j}(\varphi) \tilde{V}_{k \ell}(\varphi)-\mu^{\varepsilon} \bar{G}_{i j}\left|\bar{G}_{k \ell}\right|\right)
\end{aligned}
$$

where in general $G \mid(x)$ denotes the the coincident limit, $x^{\prime} \rightarrow x$, of the Green function $G\left(x, x^{\prime}\right)$. In $(2.13)$ we have used

$$
G_{i j}\left|=-\frac{2 \mu^{-\varepsilon}}{16 \pi^{2} \varepsilon} \tilde{V}_{i j}(\varphi)+\bar{G}_{i j}\right|
$$


where, for manifolds without boundary, $\bar{G}_{i j} \mid$ is finite for $d=4$ from (1.5), (1.6) and (1.7). Similarly for fig.1b, with its counterterms,

$$
\Gamma_{b}=\frac{\mu^{\varepsilon}}{12} \iint d v d v^{\prime} V_{i j k}^{\prime \prime \prime}(\varphi) G_{i i^{\prime}} G_{j j^{\prime}} G_{k k^{\prime}} V_{i^{\prime} j^{\prime} k^{\prime}}^{\prime \prime}\left(\varphi^{\prime}\right)-\frac{1}{16 \pi^{2} \varepsilon} \frac{1}{2} \int d v V_{i j k}^{\prime \prime \prime}(\varphi) V_{i j \ell}^{\prime \prime \prime}(\varphi) G_{k \ell} \mid .
$$

Using (1.5) and (1.8) it is straightforward to see that

$$
\begin{aligned}
\Gamma_{b} \sim \frac{\mu^{-\varepsilon}}{\left(16 \pi^{2}\right)^{2} \varepsilon} \frac{1}{24} \int d v V_{i j k}^{\prime \prime \prime}(\varphi)\left(\nabla^{2}+\frac{1}{6} R\right) V_{i j k}^{\prime \prime \prime}(\varphi) \\
+\frac{\mu^{-\varepsilon}}{\left(16 \pi^{2} \varepsilon\right)^{2}} \frac{1}{2}\left(1-\frac{1}{2} \varepsilon\right) \int d v V_{i j k}^{\prime \prime \prime}(\varphi) V_{i j \ell}^{\prime \prime \prime}(\varphi) \tilde{V}_{k \ell}(\varphi)
\end{aligned}
$$

and together with $1 / \varepsilon^{2}$ term in (2.13) this determines the required two loop counterterms for $S_{\text {c.t. }}$ as usual.

In addition it is essential to consider also the one particle reducible diagram fig.1c for which

$$
\Gamma_{c}=\frac{\mu^{\varepsilon}}{8} \iint d v d v^{\prime} \bar{G}_{j k}\left|V_{i j k}^{\prime \prime \prime}(\varphi) G_{i i^{\prime}} V_{i^{\prime} j^{\prime} k^{\prime}}^{\prime \prime \prime}\left(\varphi^{\prime}\right) \bar{G}_{j^{\prime} k^{\prime}}\right| .
$$

For no boundary $\Gamma_{c}$ is finite while here it is necessary to allow for the extra one loop boundary counterterms given by (2.8) and (2.11) which give the one particle reducible diagrams in fig.1d and

$$
\begin{aligned}
\Gamma_{d}= & \left.\frac{1}{4} \frac{1}{16 \pi^{2} \varepsilon} \iint d S d v^{\prime} V_{i j j}^{\prime \prime \prime}(\hat{\varphi}) \partial_{n} G_{i i^{\prime}}\right|_{y=0} V_{i^{\prime} j^{\prime} k^{\prime}}^{\prime \prime \prime}\left(\varphi^{\prime}\right) \bar{G}_{j^{\prime} k^{\prime}} \mid \\
& +\left.\frac{1}{8} \frac{\mu^{-\varepsilon}}{\left(16 \pi^{2} \varepsilon\right)^{2}} \iint d S d S^{\prime} V_{i j j}^{\prime \prime \prime}(\hat{\varphi}) \partial_{n} G_{i i^{\prime}} \overleftarrow{\partial}_{n}^{\prime}\right|_{y=y^{\prime}=0} V_{i^{\prime} j^{\prime} j^{\prime}}^{\prime \prime \prime}\left(\hat{\varphi}^{\prime}\right)
\end{aligned}
$$

When subdivergences are subtracted in one particle reducible amplitudes then according to the usual lore of renormalisation theory there is no remaining overall divergence. If $G_{i i^{\prime}}\left(x, x^{\prime}\right)$ in (2.17) and (2.18) is separated into a singular and a regular part then by integrating by parts from (2.17) and (2.18) we find

$$
\begin{aligned}
\left(\Gamma_{c}+\Gamma_{d}\right)^{\mathrm{reg}} & =\frac{\mu^{\varepsilon}}{8} \iint d v d v^{\prime} D_{j k} V_{i j k}^{\prime \prime \prime}(\varphi) G_{i i^{\prime}}^{\mathrm{reg}} V_{i^{\prime} j^{\prime} k^{\prime}}^{\prime \prime \prime}\left(\varphi^{\prime}\right) D_{j^{\prime} k^{\prime}}, \\
D_{j k} & =\bar{G}_{j k} \mid+\frac{\mu^{-\varepsilon}}{16 \pi^{2} \varepsilon} \delta_{j k}\left(-\delta^{\prime}(y)+\frac{1}{3} K \delta(y)\right)
\end{aligned}
$$

where $D_{j k}(x)$, as in (C.20), is the dimensionally regularised form of $G_{j k}(x, x)$ with poles in $\varepsilon$ representing both the usual local and also boundary divergences subtracted (the $\delta(y)$ term is irrelevant in this case assuming $G_{i i^{\prime}}^{\mathrm{reg}}$ also obeys Dirichlet boundary conditions and vanishes at $y=0$ or $\left.y^{\prime}=0\right)$ and hence, so long as $G_{i i^{\prime}}^{\text {reg }}\left(x, x^{\prime}\right)$ is a sufficiently smooth function on $\mathcal{M} \times \mathcal{M},(2.19)$ is finite as $\varepsilon \rightarrow 0$. However, as pointed out by Symanzik [10], for the full Green function $G_{i i^{\prime}}\left(x, x^{\prime}\right)$ there are additional divergences in $(2.17)$ not 
removed by the counterterms (2.18) and in general the manipulations leading to (2.19) are not justified. An additional complication in this case is that in (2.18) $\partial_{n} G_{i i^{\prime}} \overleftarrow{\partial}_{n}^{\prime}$ has a non integrable singularity as $\hat{\mathbf{x}}^{\prime} \rightarrow \hat{\mathbf{x}}$ which is not regularised by analytic continuation in $d$ as usual with dimensional regularisation. Nevertheless elsewhere [17] we have shown that there is a well defined prescription for handling this which we follow in detailed calculations in appendix B.

The results of appendix $\mathrm{B}$ allow the divergent terms as $\varepsilon \rightarrow 0$ on the boundary to be readily calculated for each contribution. For $\Gamma_{a}$ from $(\mathrm{B} .4,7)$, and also using $(\mathrm{B} .8,10)$, we get, keeping only terms relevant for determining $\hat{\varphi}_{\text {c.t. }}$ and $\rho$ in $(2.8)$,

$$
\Gamma_{a} \sim \frac{\mu^{-\varepsilon}}{\left(16 \pi^{2} \varepsilon\right)^{2}} \frac{1}{4} \int d S g_{i j k k}\left\{\left.\left(1-\frac{1}{2} \varepsilon\right) \partial_{n} V_{i j}^{\prime \prime}(\varphi)\right|_{y=0}-\frac{2}{3}\left(1-\frac{2}{3} \varepsilon\right) V_{i j}^{\prime \prime}(\hat{\varphi}) K\right\} .
$$

The amplitude $\Gamma_{b}$ also has, apart from (2.16), a contribution to the divergence on the boundary given by, from (B.19) and (B.20a,..f),

$$
\begin{aligned}
\Gamma_{b} \sim & -\frac{\mu^{-\varepsilon}}{\left(16 \pi^{2}\right)^{2} \varepsilon} \frac{1}{24} \int d v \nabla_{\mu} V_{i j k}^{\prime \prime \prime} \nabla^{\mu} V_{i j k}^{\prime \prime \prime} \\
& +\frac{\mu^{-\varepsilon}}{\left(16 \pi^{2} \varepsilon\right)^{2}} \frac{1}{2} \int d S\left\{\left.\left(1-\frac{1}{6} \varepsilon\right) V_{i j k}^{\prime \prime \prime}(\hat{\varphi}) \partial_{n} V_{i j k}^{\prime \prime \prime}(\varphi)\right|_{y=0}-\frac{1}{3}\left(1-\frac{1}{4} \varepsilon\right) V_{i j k}^{\prime \prime \prime}(\hat{\varphi}) V_{i j k}^{\prime \prime \prime}(\hat{\varphi}) K\right\} .
\end{aligned}
$$

For the remaining one particle reducible amplitudes we may use (B.33) to give

$\Gamma_{c}+\Gamma_{d} \sim-\frac{\mu^{-\varepsilon}}{\left(16 \pi^{2} \varepsilon\right)^{2}} \frac{1}{8} \int d S\left\{\left.(1-\varepsilon) V_{i j j}^{\prime \prime \prime}(\hat{\varphi}) \partial_{n} V_{i k k}^{\prime \prime \prime}(\varphi)\right|_{y=0}-\frac{2}{3}\left(1-\frac{1}{6} \varepsilon\right) V_{i j j}^{\prime \prime \prime}(\hat{\varphi}) V_{i k k}^{\prime \prime \prime}(\hat{\varphi}) K\right\}$.

From these results we may then read off

$$
\begin{aligned}
\hat{\varphi}_{\mathrm{c.t.} .}^{(2)} & =-\frac{1}{\left(16 \pi^{2} \varepsilon\right)^{2}}\left\{\frac{1}{4}\left(1-\frac{1}{2} \varepsilon\right) V_{i j k}^{\prime \prime \prime} g_{j k \ell \ell}+\frac{1}{2}\left(1-\frac{1}{6} \varepsilon\right) g_{i j k \ell} V_{j k \ell}^{\prime \prime \prime}-\frac{1}{8}(1-\varepsilon) g_{i j j k} V_{k \ell \ell}^{\prime \prime \prime}\right\}, \\
\rho^{(2)} & =-\frac{1}{\left(16 \pi^{2} \varepsilon\right)^{2}}\left\{\frac{1}{6}\left(1-\frac{2}{3} \varepsilon\right) g_{i j k k} V_{i j}^{\prime \prime}+\frac{1}{6}\left(1-\frac{1}{4} \varepsilon\right) V_{i j k}^{\prime \prime \prime} V_{i j k}^{\prime \prime \prime}-\frac{1}{12}\left(1-\frac{1}{6} \varepsilon\right) V_{i j j}^{\prime \prime \prime} V_{i k k}^{\prime \prime \prime}\right\} .
\end{aligned}
$$

With $S_{0}$ defined as in (1.20) we may write

$$
\begin{aligned}
S_{0}(\phi) & =S\left(\phi_{0}, g_{0}\right)+\hat{S}\left(\phi_{0}, \hat{\varphi}_{0}\right)+\mathcal{C}(\rho)+\tilde{\mathcal{C}}(\lambda, \hat{\lambda}) \\
\mathcal{C}(\rho) & =\mu^{-\varepsilon} \int_{\partial \mathcal{M}} d S \rho(\hat{\varphi}) K, \quad \tilde{\mathcal{C}}(\lambda, \hat{\lambda})=\mu^{-\varepsilon} \int_{\mathcal{M}} d v C(\lambda)+\mu^{-\varepsilon} \int_{\partial \mathcal{M}} d S \hat{C}(\hat{\lambda}),
\end{aligned}
$$

in terms of the expressions for $S, \hat{S}$ given by (1.18), (2.1). $\tilde{\mathcal{C}}$ includes all necessary $\phi, \hat{\varphi}$ independent counterterms not calculated here. To obtain (2.24) it is necessary to require*

$$
\begin{aligned}
& \phi_{0 i}=\mu^{-\frac{1}{2} \varepsilon} Z_{i j} \phi_{j}, \quad Z=(1+A)^{\frac{1}{2}}, \\
& \hat{\varphi}_{0 i}=\mu^{-\frac{1}{2} \varepsilon}\left(Z_{i j} \hat{\varphi}_{j}+Z^{-1}{ }_{i j} \hat{\varphi}_{\text {c.t.j }}\right) .
\end{aligned}
$$

* Our treatment at this point differs from Symanzik [10] and also Diehl and Dietrich [5], although results to two loops are equivalent with the latter. 
The renormalisation group equations are derived as usual by requiring invariance of physical amplitudes under rescalings of the arbitrary mass $\mu$. To obtain these it is sufficient to require

$$
\begin{aligned}
& \mu \frac{d}{d \mu} S_{0}=\left(\mathcal{D}-\int_{\mathcal{M}} d v(\hat{\gamma} \phi)_{i} \frac{\delta}{\delta \phi_{i}}\right) S_{0}=-\mathcal{C}\left(\beta^{\rho}\right)-\tilde{\mathcal{C}}\left(\beta^{\lambda}, \beta^{\hat{\lambda}}\right) \\
& \mathcal{D}=\mu \frac{\partial}{\partial \mu}+\hat{\beta}^{V} \cdot \frac{\partial}{\partial V}+\int_{\partial \mathcal{M}} d S \hat{\beta}^{\hat{\varphi}_{i}} \frac{\delta}{\delta \hat{\varphi}_{i}} .
\end{aligned}
$$

where $\hat{\beta}^{V} \cdot \partial / \partial V \equiv \hat{\beta}_{I}^{g} \partial / \partial g_{I}$ and $\beta^{\rho}, \beta^{\lambda}, \beta^{\hat{\lambda}}$ are finite. By using (2.26) with (2.5), and discarding a total functional derivative in the functional integral, it is easy to find the renormalisation group equation,

$$
\left(\mathcal{D}+\int_{\mathcal{M}} d v(\hat{\gamma} J)_{i} \frac{\delta}{\delta J_{i}}\right) W(\hat{\varphi} ; J)=\mathcal{C}\left(\beta^{\rho}\right)+\tilde{\mathcal{C}}\left(\beta^{\lambda}, \beta^{\hat{\lambda}}\right) .
$$

As is conventional for field theories without boundary in dimensional regularisation on the basis of minimal subtraction

$$
\hat{\beta}^{V}(\phi)=\varepsilon\left(V(\phi)-\frac{1}{2} V_{i}^{\prime}(\phi) \phi_{i}\right)+\beta^{V}(\phi), \quad \hat{\gamma}_{i j}=-\frac{1}{2} \varepsilon \delta_{i j}+\gamma_{i j}, \quad \hat{\beta}^{V} \cdot \frac{\partial}{\partial V} Z=Z \gamma
$$

The $\beta$ functions associated with the boundary that may be obtained by the calculations here are $\hat{\beta}^{\hat{\varphi}}$ and $\beta^{\rho}$ which from (2.26) are determined by

$$
\begin{aligned}
\left(\hat{\beta}^{V} \cdot \frac{\partial}{\partial V}+\hat{\beta}_{j}^{\hat{\varphi}_{j}}(\hat{\varphi}) \frac{\partial}{\partial \hat{\varphi}_{j}}-\frac{1}{2} \varepsilon\right) \hat{\varphi}_{0 i}(\hat{\varphi}) & =0, \quad \hat{\beta}^{\hat{\varphi}}(\hat{\varphi})=\frac{1}{2} \varepsilon \hat{\varphi}+\beta^{\hat{\varphi}}(\hat{\varphi}) \\
\left(\hat{\beta}^{V} \cdot \frac{\partial}{\partial V}+\hat{\beta}^{\hat{\varphi}_{j}}(\hat{\varphi}) \frac{\partial}{\partial \hat{\varphi}_{j}}-\varepsilon\right) \rho(\hat{\varphi}) & =-\beta^{\rho}(\hat{\varphi}) .
\end{aligned}
$$

From $(2.29 \mathrm{a})$ we therefore find to two loops, since $\beta^{V(1)}=\frac{1}{2} V_{i j}^{\prime \prime} V_{i j}^{\prime \prime} /\left(16 \pi^{2}\right)$,

$$
\begin{aligned}
\beta_{i}^{\hat{\varphi}_{i}}(\hat{\varphi}) & =-\frac{1}{16 \pi^{2}} \frac{1}{2} V_{i j j}^{\prime \prime \prime}(\hat{\varphi}) \\
& +\frac{1}{\left(16 \pi^{2}\right)^{2}} \frac{1}{12}\left\{3 V_{i j k}^{\prime \prime \prime}(\hat{\varphi}) g_{j k \ell \ell}+2 g_{i j k \ell} V_{j k \ell}^{\prime \prime \prime}(\hat{\varphi})-3 g_{i j j k} V_{k \ell \ell}^{\prime \prime \prime}(\hat{\varphi})-g_{i k \ell m} g_{j k \ell m} \hat{\varphi}_{j}\right\}
\end{aligned}
$$

using (2.11) and (2.23) with the two loop result for $Z_{i j}^{(2)}=-\frac{1}{24} g_{i k \ell m} g_{j k \ell m} /\left(16 \pi^{2}\right)^{2} \varepsilon$. As usual with dimensional regularisation the double poles in $\hat{\varphi}_{\text {c.t. }}^{(2)}$ are determined by $(2.29 \mathrm{a})$ which provides a significant consistency check on our calculations. In a similar fashion

$$
\begin{aligned}
\beta^{\rho}(\hat{\varphi}) & =-\frac{1}{16 \pi^{2}} \frac{1}{3} V_{j j}^{\prime \prime}(\hat{\varphi}) \\
& +\frac{1}{\left(16 \pi^{2}\right)^{2}} \frac{1}{36}\left\{8 g_{i j k k} V_{i j}^{\prime \prime}(\hat{\varphi})+3 V_{i j k}^{\prime \prime \prime}(\hat{\varphi}) V_{i j k}^{\prime \prime \prime}(\hat{\varphi})-V_{i j j}^{\prime \prime \prime}(\hat{\varphi}) V_{i k k}^{\prime \prime \prime}(\hat{\varphi})\right\} .
\end{aligned}
$$


For specific application of these results we focus on the $O(n)$ symmetric potential $V(\phi)=\frac{1}{24} g\left(\phi^{2}\right)^{2}+\frac{1}{2} m^{2} \phi^{2}$, for $\phi_{i}$ an $n$ component field. In this case we may write

$$
\beta^{\hat{\varphi}_{i}}(\hat{\varphi})=\hat{\eta} \hat{\varphi}_{i}, \quad \beta^{\rho}(\hat{\varphi})=\frac{1}{2} \hat{\rho} \hat{\varphi}^{2}+\hat{\rho}_{m} m^{2},
$$

so that $\hat{\eta}$ is the anomalous dimension of the boundary operator $\partial_{n} \phi$. From (2.30) and (2.31) to two loop order

$$
\begin{aligned}
& \hat{\eta}=-\frac{1}{6}(n+2) u+\frac{1}{36}(n+2) u^{2}, \\
& \hat{\rho}=-\frac{1}{9}(n+2) u+\frac{1}{54}(n+2)(n+5) u^{2}, \hat{\rho}_{m}=\frac{1}{16 \pi^{2}} \frac{n}{3}\left(-1+\frac{2}{9}(n+2) u\right),
\end{aligned}
$$

for $u=g /\left(16 \pi^{2}\right)$. For comparison with Diehl and Dietrich [5] $\hat{\eta}=\frac{1}{2} \eta_{1}+\gamma$ where for this model $\gamma_{i j}=\gamma \delta_{i j}$ and $\gamma^{(2)}=\frac{1}{36}(n+2) u^{2}$.

As an illustration of the renormalisation group equation we apply (2.27) in this $O(n)$ model to the expansion of the field operator $\phi(x)$ in the vicinity of the boundary. Defining

$$
\langle\phi(x)\rangle_{J}=\frac{\delta W(\hat{\varphi} ; J)}{\delta J(x)}, \quad \mu^{-\varepsilon}\left\langle\partial_{n} \phi(\hat{\mathbf{x}})\right\rangle_{J}=\frac{\delta W(\hat{\varphi} ; J)}{\delta \hat{\varphi}(\hat{\mathbf{x}})},
$$

then we may write, for $y \rightarrow 0$ and neglecting $m$,

$$
\langle\phi(\mathbf{x}, y)\rangle_{J} \sim C_{0}(\mu y) \hat{\varphi}(\mathbf{x})+C_{1}(\mu y) y\left\langle\partial_{n} \phi(\mathbf{x})\right\rangle_{J}+C_{2}(\mu y) y K(\mathbf{x}) \hat{\varphi}(\mathbf{x}) .
$$

From (2.27) the leading singular parts of the coefficient functions satisfy

$$
\begin{aligned}
& \left(\mu \frac{\partial}{\partial \mu}+\hat{\beta}(g) \frac{\partial}{\partial g}+\gamma(g)+\hat{\eta}(g)\right) C_{0}(\mu y)=0, \\
& \left(\mu \frac{\partial}{\partial \mu}+\hat{\beta}(g) \frac{\partial}{\partial g}+\gamma(g)-\hat{\eta}(g)\right) C_{1}(\mu y)=0, \\
& \left(\mu \frac{\partial}{\partial \mu}+\hat{\beta}(g) \frac{\partial}{\partial g}+\gamma(g)+\hat{\eta}(g)\right) C_{2}(\mu y)=-\hat{\rho}(g) C_{1}(\mu y),
\end{aligned}
$$

where manifestly for $g=0 C_{0}=C_{1}=1, C_{2}=0$. This result defines an expansion for the operator $\phi(x)$, analogous to the usual operator product expansion, in the neighbourhood of the boundary. At the infra-red fixed point corresponding to $\hat{\beta}\left(g_{*}\right)=0$

$C_{0}(\mu y) \propto(\mu y)^{-\gamma_{*}-\hat{\eta}_{*}}, \quad C_{1}(\mu y) \propto(\mu y)^{-\gamma_{*}+\hat{\eta}_{*}}, \quad C_{2}(\mu y) \sim-\frac{\hat{\rho}_{*}}{2 \hat{\eta}_{*}} C_{1}(\mu y)+\mathrm{O}\left((\mu y)^{-\gamma_{*}-\hat{\eta}_{*}}\right)$.

From (2.33), and using that with minimal subtraction the critical coupling is given by

$$
u_{*}=\frac{3}{(n+8)} \varepsilon+\frac{9}{(n+8)^{3}}(3 n+14) \varepsilon^{2}+\mathrm{O}\left(\varepsilon^{3}\right),
$$

then the critical indices are as usual expressed as an expansion in $\varepsilon$

$$
\begin{aligned}
& \hat{\gamma}_{*}=\frac{1}{4} \frac{(n+2)}{(n+8)^{2}} \varepsilon^{2}, \quad \hat{\eta}_{*}=-\frac{1}{2} \frac{n+2}{n+8} \varepsilon-\frac{1}{4} \frac{(n+2)}{(n+8)^{3}}(17 n+76) \varepsilon^{2}, \\
& \frac{\hat{\rho}_{*}}{\hat{\eta}_{*}}=\frac{2}{3}-\frac{1}{3} \frac{n+4}{n+8} \varepsilon .
\end{aligned}
$$




\section{Quantum Scalar Field Theory with Free Boundary Values}

In this section the essential quantum field theory given by the action (1.18) is extended by the surface term

$$
\hat{S}(\phi, \hat{g})=\int_{\partial \mathcal{M}} d S Q(\phi)
$$

where $Q(\phi)$, depending on couplings $\hat{g}$, is a scalar and there is no dependence on the normal derivative $\partial_{n} \phi$ as in (2.1). For renormalisability $Q(\phi)$ is at most cubic in $\phi$ although if $\phi \rightarrow-\phi$ symmetry is imposed at leading order $Q(\phi)=\mathrm{O}\left(\phi^{2}\right)$ and this is stable under renormalisation.

When $\phi$ is expanded about a background $\varphi$ then to first order

$$
S_{1}=\int_{\mathcal{M}} d v f_{i}\left(-\nabla^{2} \varphi_{i}+V_{i}^{\prime}(\varphi)\right)+\int_{\partial \mathcal{M}} d S f_{i}\left(-\partial_{n} \varphi+Q^{\prime}(\varphi)\right)_{i}
$$

so that the appropriate boundary conditions, assuming that they apply to $\phi$ as required for invariance under the shift symmetry generated by $\delta \varphi=-\delta f$, which should be imposed are now

$$
\left.\left(\partial_{n} \phi-Q^{\prime}(\phi)\right)_{i}\right|_{\partial \mathcal{M}}=0 \text {. }
$$

To second order in $f$ the action is again of the form (2.4) where $\Delta$ is now a symmetric operator acting on fields obeying the generalised Neumann boundary condition

$$
\left.\left(\partial_{n} f_{i}-Q_{i j}^{\prime \prime}(\varphi) f_{j}\right)\right|_{\partial \mathcal{M}}=0
$$

If $Q(\phi)=\frac{1}{6} \hat{g}_{i j k} \phi_{i} \phi_{j} \phi_{k}+\ldots$ there is an additional surface interaction in this case

$$
\hat{S}_{3}=-\frac{1}{12} \int_{\partial \mathcal{M}} d S \hat{g}_{i j k} f_{i} f_{j} f_{k}
$$

arising from (3.1) and also $\mathrm{O}\left(f^{2}\right)$ terms in the expansion of (3.3) but we do not undertake any calculations involving this term here.

The basic functional integral remains as in (2.5) but now integrating over fields satisfying the boundary condition (3.3) and defining now $W(J)$. The necessary local counterterms on $\mathcal{M}$ for finiteness of $W$ are just as usual for the scalar field theory without boundary, as in (2.7), but on the boundary $\partial \mathcal{M}$ they may now be written as

$$
\hat{S}_{\text {c.t. }}(\phi, g, \hat{g})=\int_{\partial \mathcal{M}} d S\left(Q_{\text {c.t. }}(\phi)+\hat{C}(\hat{\lambda})\right)
$$

where $\hat{C}(\hat{\lambda})$ represents contributions independent of $\phi$ which include all terms of the same form as those present in $\hat{C}$ for the Dirichlet case in (2.8) but also, for $\hat{g}_{i j k}=0$, any terms involving dimension two scalars formed from the metric, such as $R_{n n}, K_{i j} K^{i j}$ or $K^{2}$, and proportional to $Q^{\prime \prime}$. $Q_{\text {c.t. }}(\phi)$ then contains the remaining counterterms independent of the metric and also those linear in $K$. In the background field method $Q_{\text {c.t. }}(\varphi)$ may be calculated directly and involves, for $\hat{g}_{i j k}=0$, just terms with the general structure $V^{\prime \prime \prime}(\varphi) Q^{\prime}(\varphi)$, 
$V^{\prime \prime}(\varphi) Q^{\prime \prime}, V^{\prime \prime \prime}(\varphi)^{2} Q^{\prime \prime}, Q^{\prime \prime 3}$ while terms proportional to $K$ may contain $V^{\prime \prime}(\varphi), V^{\prime \prime \prime}(\varphi)^{2}$ or $Q^{\prime \prime 2}$. Clearly $Q_{\text {c.t. }}(\varphi)$ remains $\mathrm{O}\left(\varphi^{2}\right)$ in this case.

The one loop amplitude is still determined by the functional determinant of $\Delta$ as in (2.9), although now with the alternative boundary conditions (3.4), and (1.17) gives, after using (3.3) to eliminate $\partial_{n} \varphi$ terms,

$$
Q_{\text {c.t. }}^{(1)}=\frac{1}{16 \pi^{2} \varepsilon}\left(-\frac{1}{2} V_{i i j}^{\prime \prime \prime} Q_{j}^{\prime}+2\left(V^{\prime \prime} Q^{\prime \prime}\right)_{i i}-\frac{4}{3}\left(Q^{\prime \prime 3}\right)_{i i}+\frac{4}{3}\left(Q^{\prime \prime 2}\right)_{i i} K-\frac{1}{3} V_{i i}^{\prime \prime} K\right) .
$$

The corresponding result for $\hat{C}^{(1)}$ is easy to read off from (1.17).

At two loops the essential amplitudes are still given by (2.12), (2.14) and (2.16), if $\hat{g}_{i j k}=0$, although $G_{i j}\left(x, x^{\prime}\right)$ is now the Green function for $\Delta$ subject to boundary conditions given by (3.4). The one particle reducible diagrams in fig.1d, with the one loop boundary counterterms determined by (3.7), now give

$$
\begin{aligned}
\Gamma_{d}= & \left.\frac{1}{2} \iint d S d v^{\prime} Q_{\text {c.t. } i}^{(1) \prime}(\varphi) G_{i i^{\prime}}\right|_{y=0} V_{i^{\prime} j^{\prime} k^{\prime}}^{\prime \prime \prime}\left(\varphi^{\prime}\right) \bar{G}_{j^{\prime} k^{\prime}} \mid \\
& +\left.\frac{\mu^{-\varepsilon}}{2} \iint d S d S^{\prime} Q_{\text {c.t. } i}^{(1) \prime}(\varphi) G_{i i^{\prime}} Q_{\text {c.t. } i^{\prime}}^{(1) \prime}\left(\varphi^{\prime}\right)\right|_{y=y^{\prime}=0} .
\end{aligned}
$$

In a similar fashion to (2.19), if $G_{i i^{\prime}}\left(x, x^{\prime}\right) \rightarrow G_{i i^{\prime}}^{\mathrm{reg}}\left(x, x^{\prime}\right)$ which is assumed to satisfy the same boundary conditions, then combining (2.17) and (3.8) gives the finite expression

$$
\begin{aligned}
\left(\Gamma_{c}+\Gamma_{d}\right)^{\mathrm{reg}} & =\frac{\mu^{\varepsilon}}{8} \iint d v d v^{\prime} D_{j k} V_{i j k}^{\prime \prime \prime}(\varphi) G_{i i^{\prime}}^{\mathrm{reg}} V_{i^{\prime} j^{\prime} k^{\prime}}^{\prime \prime \prime}\left(\varphi^{\prime}\right) D_{j^{\prime} k^{\prime}}, \\
D_{j k} & =\bar{G}_{j k} \mid+\frac{\mu^{-\varepsilon}}{16 \pi^{2} \varepsilon}\left(\delta_{j k} \delta^{\prime}(y)+\left(4 Q_{j k}^{\prime \prime}-\delta_{j k} \frac{5}{3} K\right) \delta(y)\right),
\end{aligned}
$$

since $D_{j k}$ is in accord with (C.20) for this case as well. Since $Q_{\text {c.t. }}^{(1)}(\phi)$ is quadratic in $\phi$ there is an additional diagram fig.1e which corresponds to

$$
\begin{aligned}
\Gamma_{e} & =-\frac{\mu^{-\varepsilon}}{2} \int d S Q_{\mathrm{c.t.} .}^{(1) \prime \prime} G_{i j} \mid \\
& =-\frac{1}{4} \frac{\mu^{-\varepsilon}}{16 \pi^{2} \varepsilon} \int d v g_{i j k \ell}\left(\delta_{k \ell} \delta^{\prime}(y)+\left(4 Q_{k \ell}^{\prime \prime}-\delta_{k \ell} \frac{5}{3} K\right) \delta(y)\right) G_{i j} \mid
\end{aligned}
$$

The results of appendix B again allow the divergent contributions on the boundary to be calculated for terms independent of and linear in $K$. From $($ B.4,6) we get

$$
\begin{aligned}
\Gamma_{a}+\Gamma_{e} \sim \frac{\mu^{-\varepsilon}}{\left(16 \pi^{2} \varepsilon\right)^{2}} & \int d S\left\{g _ { i j k k } \left(2\left(Q^{\prime \prime 3}\right)_{i j}-(1-\varepsilon)\left(Q^{\prime \prime} V^{\prime \prime}\right)_{i j}\right.\right. \\
& \left.-\frac{1}{8}(2+\varepsilon) V_{i j \ell}^{\prime \prime \prime} Q_{\ell}^{\prime}-\frac{2}{3}(1-\varepsilon)\left(Q^{\prime \prime 2}\right)_{i j} K-\frac{1}{2}\left(1+\frac{2}{9} \varepsilon\right) V_{i j}^{\prime \prime} K\right) \\
& \left.+g_{i j k \ell}\left((3-\varepsilon) Q_{i j}^{\prime \prime} V_{k \ell}^{\prime \prime}-4 Q_{i j}^{\prime \prime}\left(Q^{\prime \prime 2}\right)_{k \ell}+\frac{4}{3}\left(2-\frac{1}{3} \varepsilon\right) Q_{i j}^{\prime \prime} Q_{k \ell}^{\prime \prime} K\right)\right\} .
\end{aligned}
$$


Also from (B.19) and (B.20a,..f)

$$
\begin{aligned}
\Gamma_{b} \sim- & \frac{\mu^{-\varepsilon}}{\left(16 \pi^{2}\right)^{2} \varepsilon} \frac{1}{24} \int d v \nabla_{\mu} V_{i j k}^{\prime \prime \prime} \nabla^{\mu} V_{i j k}^{\prime \prime \prime} \\
+\frac{\mu^{-\varepsilon}}{\left(16 \pi^{2} \varepsilon\right)^{2}} \int d S\{ & -\frac{1}{2}(1-\varepsilon) V_{i j k}^{\prime \prime \prime} g_{i j k \ell} Q_{\ell}^{\prime}+\left(1-\frac{1}{2} \varepsilon-\frac{1}{6} \pi^{2} \varepsilon\right) V_{i k \ell}^{\prime \prime \prime} V_{j k \ell}^{\prime \prime \prime} Q_{i j}^{\prime \prime} \\
& \left.-\frac{1}{6}\left(1-\frac{1}{12} \varepsilon-\frac{1}{3} \pi^{2} \varepsilon\right) V_{i j k}^{\prime \prime \prime} V_{i j k}^{\prime \prime \prime} K\right\} .
\end{aligned}
$$

For the remaining one particle reducible amplitudes we may use $(B .27,28,31)$ to give

$$
\begin{aligned}
\Gamma_{c}+\Gamma_{d} \sim \frac{\mu^{-\varepsilon}}{\left(16 \pi^{2} \varepsilon\right)^{2}} \int d S\{ & \frac{1}{8}(1+\varepsilon) V_{i i k}^{\prime \prime \prime} g_{j j k \ell} Q_{\ell}^{\prime}+\frac{1}{8} V_{i i k}^{\prime \prime \prime} V_{j j \ell}^{\prime \prime \prime} Q_{k \ell}^{\prime \prime} \\
& \left.-\frac{1}{2} V_{i i \ell}^{\prime \prime \prime} V_{j k \ell}^{\prime \prime \prime} Q_{j k}^{\prime \prime}+\frac{1}{12}\left(1+\frac{1}{6} \varepsilon\right) V_{i i k}^{\prime \prime \prime} V_{j j k}^{\prime \prime \prime} K\right\} .
\end{aligned}
$$

Combining these we therefore obtain the somewhat lengthy result

$$
\begin{aligned}
Q_{\text {c.t. }}^{(2)}=\frac{1}{\left(16 \pi^{2} \varepsilon\right)^{2}}\{ & g_{i j k k}\left(2\left(Q^{\prime \prime 3}\right)_{i j}-(1-\varepsilon)\left(Q^{\prime \prime} V^{\prime \prime}\right)_{i j}\right. \\
& \left.\quad-\frac{1}{8}(2+\varepsilon) V_{i j \ell}^{\prime \prime \prime} Q_{\ell}^{\prime}-\frac{2}{3}(1-\varepsilon)\left(Q^{\prime \prime 2}\right)_{i j} K-\frac{1}{2}\left(1+\frac{2}{9} \varepsilon\right) V_{i j}^{\prime \prime} K\right) \\
& +g_{i j k \ell}\left((3-\varepsilon) Q_{i j}^{\prime \prime} V_{k \ell}^{\prime \prime}-4 Q_{i j}^{\prime \prime}\left(Q^{\prime \prime 2}\right)_{k \ell}+\frac{4}{3}\left(2-\frac{1}{3} \varepsilon\right) Q_{i j}^{\prime \prime} Q_{k \ell}^{\prime \prime} K\right) \\
& +\frac{1}{8}(1+\varepsilon) V_{i i k}^{\prime \prime \prime} g_{j j k \ell} Q_{\ell}^{\prime}+\frac{1}{8} V_{i i k}^{\prime \prime \prime} V_{j j \ell}^{\prime \prime \prime} Q_{k \ell}^{\prime \prime}-\frac{1}{2} V_{i i \ell}^{\prime \prime \prime} V_{j k \ell}^{\prime \prime \prime} Q_{j k}^{\prime \prime} \\
& +\frac{1}{12}\left(1+\frac{1}{6} \varepsilon\right) V_{i i k}^{\prime \prime \prime} V_{j j k}^{\prime \prime \prime} K-\frac{1}{2}(1-\varepsilon) V_{i j k}^{\prime \prime \prime} g_{i j k \ell} Q_{\ell}^{\prime} \\
& \left.+\left(1-\frac{1}{2} \varepsilon-\frac{1}{6} \pi^{2} \varepsilon\right) V_{i k \ell}^{\prime \prime \prime} V_{j k \ell}^{\prime \prime \prime} Q_{i j}^{\prime \prime}-\frac{1}{6}\left(1-\frac{1}{12} \varepsilon-\frac{1}{3} \pi^{2} \varepsilon\right) V_{i j k}^{\prime \prime \prime} V_{i j k}^{\prime \prime \prime} K\right\} .
\end{aligned}
$$

To obtain the renormalisation group equations we define, as in (2.24),

$$
\begin{aligned}
S_{0}(\phi) & =S\left(\phi_{0}, g_{0}\right)+\hat{S}\left(\phi_{0}, \hat{g}_{0}\right)+\tilde{\mathcal{C}}(\lambda, \hat{\lambda}), \\
Q_{0}\left(\phi_{0}\right) & =\mu^{-\varepsilon}\left(Q(\phi)+Q_{\text {c.t. }}(\phi)\right),
\end{aligned}
$$

for $\tilde{\mathcal{C}}(\lambda, \hat{\lambda})$ formed from the counterterms $C(\lambda)$ and $\hat{C}(\hat{\lambda})$ in this case. Since $Q_{\text {c.t. contains }}$ terms involving $K$, which is in general $\hat{\mathbf{x}}$ dependent, it is necessary to regard the couplings $\hat{g}$ in $Q$ as also $\hat{\mathbf{x}}$ dependent to ensure multiplicative renormalisability or that $Q_{0}$ is obtained from $Q$ by $\hat{g} \rightarrow \hat{g}_{0}$. In this case instead of (2.26) we may then define the $\beta$ functions by

$$
\begin{gathered}
\mu \frac{d}{d \mu} S_{0}=\left(\mathcal{D}-\int_{\mathcal{M}} d v(\hat{\gamma} \phi)_{i} \frac{\delta}{\delta \phi_{i}}\right) S_{0}=-\tilde{\mathcal{C}}\left(\beta^{\lambda}, \beta^{\hat{\lambda}}\right) \\
\mathcal{D}=\mu \frac{\partial}{\partial \mu}+\hat{\beta}^{V} \cdot \frac{\partial}{\partial V}+\hat{\beta}^{Q} \cdot \frac{\partial}{\partial Q} .
\end{gathered}
$$

In particular $\hat{\beta}^{Q}$ is determined by

$$
\left(\hat{\beta}^{V} \cdot \frac{\partial}{\partial V}+\hat{\beta}^{Q} \cdot \frac{\partial}{\partial Q}-(\hat{\gamma} \phi)_{i} \frac{\partial}{\partial \phi_{i}}-\varepsilon\right) Q_{0}=0, \hat{\beta}^{Q}(\phi)=\varepsilon\left(Q(\phi)-\frac{1}{2} Q_{i}^{\prime}(\phi) \phi_{i}\right)+\beta^{Q}(\phi) .
$$


Although $Q_{0}, \beta^{Q}$ are both given as linear in $K$ it is possible to generate $K^{2}$ terms in (3.17), with the assumed structure of counterterms even if $Q^{\prime \prime \prime}=0$, but these may be neglected since they are not calculated here. To two loops the $\beta$ function becomes

$$
\begin{aligned}
\beta^{Q}-Q_{i}^{\prime}(\gamma \phi)_{i}= & \frac{1}{16 \pi^{2}}\left\{-\frac{1}{2} V_{i i j}^{\prime \prime \prime} Q_{j}^{\prime}+2\left(V^{\prime \prime} Q^{\prime \prime}\right)_{i i}-\frac{4}{3}\left(Q^{\prime \prime 3}\right)_{i i}+\frac{1}{3}\left(4\left(Q^{\prime \prime 2}\right)_{i i}-V_{i i}^{\prime \prime}\right) K\right\} \\
& +\frac{1}{\left(16 \pi^{2}\right)^{2}}\left\{g_{i j k k}\left(2\left(Q^{\prime \prime} V^{\prime \prime}\right)_{i j}-\frac{1}{4} V_{i j \ell}^{\prime \prime \prime} Q_{\ell}^{\prime}\right)-2 g_{i j k \ell} Q_{i j}^{\prime \prime} V_{k \ell}^{\prime \prime}\right. \\
& +\frac{1}{4} V_{i i k}^{\prime \prime \prime} g_{j j k \ell} Q_{\ell}^{\prime}+V_{i j k}^{\prime \prime \prime} g_{i j k \ell} Q_{\ell}^{\prime}-\left(1+\frac{1}{3} \pi^{2}\right) V_{i k \ell}^{\prime \prime \prime} V_{j k \ell}^{\prime \prime \prime} Q_{i j}^{\prime \prime} \\
& +\frac{2}{9} g_{i j k k}\left(6\left(Q^{\prime \prime 2}\right)_{i j}-V_{i j}^{\prime \prime}\right) K-\frac{8}{9} g_{i j k \ell} Q_{i j}^{\prime \prime} Q_{k \ell}^{\prime \prime} K \\
& \left.+\frac{1}{36}\left(V_{i i k}^{\prime \prime \prime} V_{j j k}^{\prime \prime \prime}+\left(1+4 \pi^{2}\right) V_{i j k}^{\prime \prime \prime} V_{i j k}^{\prime \prime \prime}\right) K\right\} .
\end{aligned}
$$

Instead of (2.27) the renormalisation group equation is now

$$
\left(\mathcal{D}+\int_{\mathcal{M}} d v(\hat{\gamma} J)_{i} \frac{\delta}{\delta J_{i}}\right) W(J)=\tilde{\mathcal{C}}\left(\beta^{\lambda}, \beta^{\hat{\lambda}}\right)
$$

with $\mathcal{D}$ as in (3.16).

For the $O(n)$ symmetric case considered at the end of the last section, where there is just a single coupling $g$, we may write

$$
\begin{aligned}
Q(\phi) & =c \frac{1}{2} \phi^{2}-\hat{h}_{i} \phi_{i}, \quad \beta^{Q}(\phi)=\beta_{c} \frac{1}{2} \phi^{2}-\hat{\eta} \hat{h}_{i} \phi_{i}+\hat{\chi}, \\
\beta_{c} & =\eta_{c} c+\hat{\rho} K, \quad \hat{\chi}=\hat{\sigma}_{m} c m^{2}+\hat{\sigma}_{c} c^{3}+\left(\hat{\rho}_{m} m^{2}+\hat{\rho}_{c} c^{2}\right) K+\hat{\omega} \partial_{n} m^{2},
\end{aligned}
$$

where the term proportional to $\partial_{n} m^{2}$ is present if $m^{2}$ is dependent on $x$ and is discussed further in the next section. The renormalisation group equation (3.19), setting the curvature on $\mathcal{M}$ to zero, becomes

$$
\begin{aligned}
\left(\mu \frac{\partial}{\partial \mu}\right. & \left.+\hat{\beta} \frac{\partial}{\partial g}+\int_{\mathcal{M}} d v\left(\gamma_{m} m^{2} \frac{\delta}{\delta m^{2}}+\hat{\gamma} J_{i} \frac{\delta}{\delta J_{i}}\right)+\int_{\partial \mathcal{M}} d S\left(\beta_{c} \frac{\delta}{\delta c}+\left(\frac{1}{2} \varepsilon+\hat{\eta}\right) \hat{h}_{i} \frac{\delta}{\delta \hat{h}_{i}}\right)\right) W(J) \\
& =\mu^{-\varepsilon} \int_{\mathcal{M}} d v \frac{1}{2} p m^{4}+\mu^{-\varepsilon} \int_{\partial \mathcal{M}} d S\left(\hat{\chi}+C\left(\beta^{\hat{\lambda}}\right)\right)
\end{aligned}
$$

where $\hat{\beta}^{V}(\phi)=\frac{1}{24} \hat{\beta} \phi^{4}+\frac{1}{2} \gamma_{m} m^{2} \phi^{2}+\frac{1}{2} p m^{4}$.

The results of our calculations then imply to two loop order

$$
\begin{aligned}
& \eta_{c}=\frac{1}{3}(n+2) u+\frac{1}{18}\left(1-4 \pi^{2}\right)(n+2) u^{2}, \quad \hat{\eta}=-\frac{1}{6}(n+2) u+\frac{13}{36}(n+2) u^{2}, \\
& \frac{\hat{\rho}}{\eta_{c}}=-\frac{1}{3}\left(1+\frac{1}{6} n u\right), \quad \hat{\rho}_{m}=-\frac{1}{16 \pi^{2}} \frac{n}{3}\left(1+\frac{2}{9}(n+2) u\right), \\
& \hat{\rho}_{c}=\frac{1}{16 \pi^{2}} \frac{4 n}{3}\left(1+\frac{1}{9}(n+2) u\right), \quad \hat{\sigma}_{m}=\frac{1}{16 \pi^{2}} 2 n, \quad \hat{\sigma}_{c}=-\frac{1}{16 \pi^{2}} \frac{4 n}{3},
\end{aligned}
$$

where $\eta_{c}$ and $\hat{\eta}$ are in accord with the results of Diehl and Dietrich [5] with $\eta_{c} \rightarrow \eta_{c}, \hat{\eta}=$ $\frac{1}{2} \eta_{1}+\gamma$. In a general regularisation scheme $c$ is linearly divergent but as usual such contributions are absent with dimensional regularisation. 


\section{Conformal Invariance and Consistency Relations}

The essential new results of the previous two sections has been to calculate, to two loops, those counterterms necessary for a renormalisable quantum scalar field theory which depend on the extrinsic curvature $K$ of the boundary. In this section we show how consistency conditions resulting from considering arbitrary local Weyl rescalings of the metric on $\mathcal{M}$ and hence also of the induced metric on $\partial \mathcal{M}$ may determine the $K$ dependent counterterms without undertaking any calculations beyond those for a flat spatial background and with a non curved boundary. The method is an extension of that used to derive consistency relations which determine the dependence of counterterms on the Riemann curvature for general renormalisable field theories on manifolds without a boundary [18].

Under an infinitesimal Weyl rescaling the essential results here are

$$
\delta_{\sigma} g^{\mu \nu}=2 \sigma g^{\mu \nu}, \quad \delta_{\sigma} R=2 \sigma R+2(d-1) \nabla^{2} \sigma, \quad \delta_{\sigma} n^{\mu}=\sigma n^{\mu}, \quad \delta_{\sigma} K=\sigma K+(d-1) \partial_{n} \sigma .
$$

The consistency conditions are derived by considering relations involving the finite local scalar composite operators constructed from the basic fields. To define these the essential couplings $g_{I}, \hat{g}_{I}$ parameterising the theory are extended to arbitrary local functions $g_{I}(x)$, as well as $\hat{g}_{I}(\hat{\mathbf{x}})$, or effectively $V \rightarrow V(\phi, x), Q \rightarrow Q(\phi, \hat{\mathbf{x}})$, so that local operators may be obtained by functional differentiation. The quantum field theory remains finite, in renormalised perturbation theory, so long as appropriate additional counterterms depending on derivatives of the couplings are introduced. In particular on $\partial \mathcal{M}$ there are possible counterterms depending on $\partial_{n} g_{I}$.

Here we consider initially the basic scalar quantum field theory with classical action (1.18) with Neumann boundary conditions so that there is also a surface term as in (3.1). If $V(\phi)=\sum_{I} g_{I} v_{I}(\phi)$ and $Q(\phi)=\sum_{I} \hat{g}_{I} q_{I}(\phi)$, for some basis of quartic polynomials $v_{I}(\phi)$ and quadratic polynomials $q_{I}(\phi)$, then we define $\Delta g_{I}$ in terms of $\Delta V(\phi)=4 V(\phi)-V_{i}^{\prime}(\phi) \phi_{i}$ and $\Delta \hat{g}_{I}$ by $\Delta Q(\phi)=3 Q(\phi)-Q_{i}^{\prime}(\phi) \phi_{i}$ so that if $\Delta g_{I}=\Delta_{I} g_{I}$ and $\Delta \hat{g}_{I}=\hat{\Delta}_{I} \hat{g}_{I}$ then $\Delta_{I}$, $\hat{\Delta}_{I}$ are the dimensions of the associated couplings. Defining

$\Delta_{\sigma}^{W}=\int_{\mathcal{M}} d v \sigma\left(2 g^{\mu \nu} \frac{\delta}{\delta g^{\mu \nu}}+\Delta g_{I} \frac{\delta}{\delta g_{I}}\right), \quad \hat{\Delta}_{\sigma}^{W}=\int_{\partial \mathcal{M}} d S \sigma \Delta \hat{g}_{I} \frac{\delta}{\delta \hat{g}_{I}}, \quad \Delta_{\sigma}^{\phi}=\int_{\mathcal{M}} d v \sigma \phi_{i} \frac{\delta}{\delta \phi_{i}}$,

then for $d=4$ by simple scale invariance it is easy to see from (1.18) and (3.1) that

$$
\left(\Delta_{\sigma}^{W}+\hat{\Delta}_{\sigma}^{W}+\Delta_{\sigma}^{\phi}\right)(S+\hat{S})=-\frac{1}{2} \int_{\mathcal{M}} d v \phi^{2} \nabla^{2} \sigma-\frac{1}{2} \int_{\partial \mathcal{M}} d S \phi^{2} \partial_{n} \sigma .
$$

The $\nabla^{2} \sigma, \partial_{n} \sigma$ terms appearing on the r.h.s. of (4.3) may be cancelled by introducing appropriate contributions involving $R \phi^{2}$ in $S$ and $K \phi^{2}$ in $\hat{S}$ which ensures a classically Weyl invariant theory under local rescalings of the metric as in (4.1) if there are no couplings with dimension, $\Delta g_{I}=\Delta \hat{g}_{I}=0$. Later we show how this may be extended to the quantum theory at a critical point $\hat{\beta}(g)=0$.

In the full quantum field theory, for general $d$, where $S+\hat{S} \rightarrow S_{0}$ the corresponding equations representing local changes in scale involve the $\beta$ functions of the various couplings 
and the anomalous dimension of $\phi$. Defining in addition to (4.2)

$$
\Delta_{\sigma}^{\beta}=\int_{\mathcal{M}} d v \sigma \hat{\beta}_{I}^{g} \frac{\delta}{\delta g_{I}}, \quad \hat{\Delta}_{\sigma}^{\beta}=\int_{\partial \mathcal{M}} d S \sigma \hat{\beta}_{I}^{\hat{g}} \frac{\delta}{\delta \hat{g}_{I}}, \quad \Delta_{\sigma}^{\hat{\gamma} \phi}=\int_{\mathcal{M}} d v \sigma(\hat{\gamma} \phi)_{i} \frac{\delta}{\delta \phi_{i}} .
$$

then the local renormalisation equation may be expanded in the form

$$
\begin{array}{r}
\left(\Delta_{\sigma}^{W}+\hat{\Delta}_{\sigma}^{W}+\Delta_{\sigma}^{\phi}+\Delta_{\sigma}^{\hat{\gamma} \phi}-\Delta_{\sigma}^{\beta}-\hat{\Delta}_{\sigma}^{\beta}\right) S_{0}=\mu^{-\varepsilon} \int_{\mathcal{M}} d v \sigma C\left(\beta^{\lambda}\right)+\mu^{-\varepsilon} \int_{\partial \mathcal{M}} d S \sigma \hat{C}\left(\beta^{\hat{\lambda}}\right) \\
-\mu^{-\varepsilon} \int_{\mathcal{M}} d v\left(\partial_{\mu} \sigma \mathcal{Z}^{\mu}+\nabla^{2} \sigma \mathcal{T}\right)-\mu^{-\varepsilon} \int_{\partial \mathcal{M}} d S \partial_{n} \sigma \mathcal{Y}
\end{array}
$$

where $\mathcal{Z}^{\mu}, \mathcal{T}$ and $\mathcal{Y}$ may depend locally on $\phi$ but not its derivatives. Since $g_{I}$ is required to be $x$ dependent for a consistent treatment it is necessary to include additional terms in $\beta^{V}(\phi)=\sum_{I} \beta_{I}^{g} v_{I}(\phi)$, and also in $C\left(\beta^{\lambda}\right)$, depending on $\partial_{\mu} g_{I}$ which have been discussed in detail in ref. [18]. To absorb all counterterms by a redefinition of couplings in this case it is also necessary to introduce an external gauge field coupled to $\phi$ but this is omitted here since it is unnecessary for obtaining the essential results of our discussion. When $\sigma$ is constant then (4.3) is equivalent to combining the usual renormalisation group equation (3.16) describing variations in $\mu$ together with a simple scaling relation. Since $S_{0}$ defines a finite theory for arbitrary $g_{\mu \nu}(x)$ and $g_{I}(x), \hat{g}_{I}(\hat{\mathbf{x}})$ the l.h.s. of (4.3) represents a linear combination of finite local operators and hence the new quantities $\mathcal{Z}^{\mu}, \mathcal{T}$ and on the boundary $\mathcal{Y}$ appearing on the r.h.s. of (4.3) must therefore also be expressible in terms of finite local operators. In the first paper of [18] $\mathcal{Z}^{\mu}$ and $\mathcal{T}$ have been calculated to two loops and consistency relations following from the requirement of finiteness have been analysed.

Here we consider just the surface contributions depending on $\partial_{n} \sigma$. To calculate $\mathcal{Y}$ in (4.5) it is convenient to decompose the counterterms for $Q$ in the form

$$
Q_{\text {c.t. }}(\phi)=q(\phi)+\rho(\phi) K+w_{I}(\phi) \partial_{n} g_{I},
$$

where the last term is new in this case when the couplings are allowed to be $x$ dependent. It is then easy to see from the defining equations (4.3) and (4.1) that in general

$$
\mathcal{Y}(\phi)=u(\phi)-(3-\varepsilon) \rho(\phi)-w_{I}(\phi)\left(\Delta g_{I}-\hat{\beta}_{I}^{g}\right)
$$

if $u(\phi)$ is the surface contribution resulting when the volume integrals are integrated by parts to achieve the desired form (4.5).

The results for $\rho$ to two loops are contained in (3.7) and (3.14). For $\omega_{I}(\phi)$ then a straightforward extension of the calculations in section 3 gives

$$
\begin{aligned}
w_{I}^{(1)} \partial_{n} g_{I}= & -\frac{1}{16 \pi^{2} \varepsilon} \frac{1}{2} \partial_{n}^{\prime} V_{i i}^{\prime \prime} \\
w_{I}^{(2)} \partial_{n} g_{I}= & \frac{1}{\left(16 \pi^{2} \varepsilon\right)^{2}}\left\{-\frac{1}{4}\left(1+\frac{1}{2} \varepsilon\right) g_{i j k k} \partial_{n}^{\prime} V_{i j}^{\prime \prime}+\frac{1}{8}(1+\varepsilon) V_{i i k}^{\prime \prime \prime} \partial_{n}^{\prime} V_{j j k}^{\prime \prime \prime}\right. \\
& \left.\quad-\frac{1}{2}(1-\varepsilon) V_{i j k}^{\prime \prime \prime} \partial_{n}^{\prime} V_{i j k}^{\prime \prime \prime}-\frac{3}{4} \partial_{n} g_{i j k k} V_{i j}^{\prime \prime}+(1+\varepsilon) \partial_{n} g_{i j k k}\left(Q^{\prime \prime 2}\right)_{i j}\right\},
\end{aligned}
$$


where $\partial_{n}^{\prime}=n^{\mu} \partial_{\mu}^{\prime}$ with $\partial_{\mu}^{\prime}$ denoting the derivative at constant $\phi$. To obtain $u(\phi)$ then taking into account (4.3) and the derivative terms which appear at two loops in the volume integral as in (3.12) to this order gives

$$
u(\phi)=\frac{1}{2}\left(1-\frac{1}{2} \varepsilon\right) \phi^{2}-\frac{1}{\left(16 \pi^{2}\right)^{2} \varepsilon} \frac{1}{24}\left(1+\frac{1}{2} \varepsilon\right) V_{i j k}^{\prime \prime \prime}(\phi) V_{i j k}^{\prime \prime \prime}(\phi)+\ldots .
$$

As already remarked the consistency conditions stem from the fact that each term in (4.5) must separately be finite as operators, or on insertion in the regularised functional integral defined by $S_{0}$. Since arbitrary finite local composite operators corresponding to $h(\phi)=h_{I} q_{I}(\phi)$ on $\partial \mathcal{M}$, for $h(\phi)$ at most quadratic in $\phi$, may be defined by

$$
h_{I} \frac{\delta}{\delta \hat{g}_{I}(\hat{\mathbf{x}})} S_{0}
$$

then we may write

$$
\mu^{-\epsilon} \mathcal{Y}(\phi)=h_{I} \frac{\delta}{\delta \hat{g}_{I}} S_{0}
$$

for suitable $h(\phi)$, non singular as $\varepsilon \rightarrow 0$, in this case. As a consequence of minimal subtraction (4.11) all terms involving poles in $\varepsilon$ in $\mathcal{Y}(\phi)$ are determined in terms of $h(\phi)$ and the $Q$ dependent counterterms in $S_{0} . h(\phi)$ also is determined by the $\mathrm{O}(1)$ and $\mathrm{O}(\varepsilon)$ terms in (4.11). It is easy to read off from (4.7) and (4.9) the lowest order contributions

$$
h(\phi)=\frac{1}{2}\left(1-\frac{1}{2} \varepsilon\right) \phi^{2}+\frac{1}{16 \pi} \frac{2}{3}\left(V_{i i}^{\prime \prime}(\phi)-Q_{i j}^{\prime \prime}(\phi) Q_{i j}^{\prime \prime}(\phi)\right)+\ldots
$$

where the one loop contribution arises from $\varepsilon \rho^{(1)}(\phi)$ in $(4.7)$ and also from the $-\frac{1}{4} \varepsilon \phi^{2}$ term in $h(\phi)$ in conjunction with $q^{(1)}$. (4.11) then provides the crucial consistency relation which in effect determines $\rho$ in (4.6) in terms of lower order results in the loop expansion. We have verified that this confirms the one and two loop results of section 3 . As an example from (3.7)

$$
3 \rho^{(1)}=\frac{1}{16 \pi^{2} \varepsilon}\left(4\left(Q^{\prime \prime 2}\right)_{i i}-V_{i i}^{\prime \prime}\right), \quad q^{(1)}=\frac{1}{16 \pi^{2} \varepsilon}\left(2\left(V^{\prime \prime} Q^{\prime \prime}\right)_{i i}-\frac{1}{2} V_{i i j}^{\prime \prime} Q_{j}^{\prime}-\frac{4}{3}\left(Q^{\prime \prime 3}\right)_{i i}\right)
$$

and from (4.8a)

$$
w_{I}^{(1)} \Delta g_{I}=-\frac{1}{16 \pi^{2} \varepsilon}\left(V_{i i}^{\prime \prime}-\frac{1}{2} V_{i i j}^{\prime \prime \prime} \phi_{j}\right),
$$

so that it is easy to verify (4.11) at the lowest non trivial order since

$$
3 \rho^{(1)}+w_{I}^{(1)} \Delta g_{I}=-h_{I}^{0} \frac{\partial}{\partial \hat{g}_{I}} q^{(1)}
$$

for $h^{0}(\phi)=\frac{1}{2} \phi^{2}$. The same procedure has also been used to check $\rho^{(2)}$ using $h$ in $(4.12)$ although many more terms contribute. 
From (4.5) we may derive a local renormalisation group equation for the functional $W$. Restricting to the $O(n)$ symmetric model considered at the end of sections 2 and 3 and also assuming the coupling $g=g_{*}$ is at the critical point where $\hat{\beta}\left(g_{*}\right)=0$ then defining

$$
\begin{aligned}
\Delta_{\sigma}= & \int_{\mathcal{M}} d v \sigma\left(2 g^{\mu \nu} \frac{\delta}{\delta g^{\mu \nu}}+\left(2-\gamma_{m *}\right) m^{2} \frac{\delta}{\delta m^{2}}+\left(3-\varepsilon-\hat{\gamma}_{*}\right) J_{i} \frac{\delta}{\delta J_{i}}\right) \\
& +\int_{\partial \mathcal{M}} d S \sigma\left(\left(1-\eta_{c *}\right) c \frac{\delta}{\delta c}+\left(2-\frac{1}{2} \varepsilon-\hat{\eta}_{*}\right) \hat{h}_{i} \frac{\delta}{\delta \hat{h}_{i}}\right) \\
\Delta_{\sigma}^{\prime}= & \int_{\mathcal{M}} d v\left(\nabla^{2} \sigma+\frac{1}{2} \sigma R^{\prime} \gamma_{m *}\right) \tau \frac{\delta}{\delta m^{2}}+\int_{\partial \mathcal{M}} d S\left(\partial_{n} \sigma+\sigma K^{\prime} \eta_{c *}\right) \hat{\theta} \frac{\delta}{\delta c}
\end{aligned}
$$

where

$$
R^{\prime}=\frac{1}{d-1} R, \quad K^{\prime}=\frac{1}{d-1} K
$$

the local renormalisation group equation has the general form

$$
\left(\Delta_{\sigma}+\Delta_{\sigma}^{\prime}\right) W\left(m^{2}, c, J, \hat{h}\right)=X\left(m^{2}-\frac{1}{2} \tau R^{\prime}, c-\hat{\theta} K^{\prime}\right)
$$

with $X$ a volume or surface integral of local scalar functions of $m^{2}$ and $c$ proportional to $\sigma$ or its derivatives of dimension 4 or 3 respectively. The arguments of $X$ are written in the form shown in (4.15), without any loss of generality, for later convenience. From (4.5) and (4.10) the coefficients $\hat{\theta}, \iota_{c}, \iota_{m}$ are determined by writing $h(\phi)$ as

$$
h(\phi)=\frac{1}{2} \hat{\theta} \phi^{2}+\iota_{c} c^{2}+\iota_{m} m^{2}
$$

with the coupling $g$ evaluated at the critical point. Using $(3.20,21)$ with $g=g_{*}$ and the consistency relations (4.7), together with previous results in the case of theories without boundary [18], gives

$$
\begin{aligned}
X\left(m^{2}, c\right)=-\mu^{-\varepsilon} \int_{\mathcal{M}} d v\left\{\sigma\left(\frac{1}{2} p m^{4}-\beta_{a} F-\beta_{b} G\right)+\left(\nabla^{2} \sigma-\frac{1}{2} \sigma R^{\prime}\left(\gamma_{m *}-\varepsilon\right)\right) k m^{2}\right\} \\
-\mu^{-\varepsilon} \int_{\partial \mathcal{M}} d S\left\{\sigma\left(\hat{\sigma}_{c} c^{3}+\hat{\sigma}_{m} c m^{2}+\hat{\rho}_{m}^{\prime} K^{\prime} m^{2}+\hat{\omega} \partial_{n} m^{2}\right)\right. \\
\left.\quad-\partial_{n} \sigma \iota_{m} m^{2}-\left(\partial_{n} \sigma-\sigma K^{\prime}\left(2 \eta_{c *}-\varepsilon\right)\right) \iota_{c} c^{2}+\ldots\right\},
\end{aligned}
$$

with

$$
\hat{\rho}_{m}^{\prime}=(d-1) \hat{\rho}_{m}+\hat{\theta} \hat{\sigma}_{m}=\left(\gamma_{m *}-\varepsilon\right) \iota_{m}-\left(2-\gamma_{m *}\right) \hat{\omega} .
$$

The neglected terms in the surface integral in (4.17) are linear in $c$ and involve invariants constructed from $K_{i j}$ and the Riemann tensor on $\mathcal{M}$ for which the corresponding counterterms are not calculated here.

The restricted form of the functional operator $\Delta_{\sigma}^{\prime}$ in (4.14) and also $X$ in (4.17) is a consequence of the consistency relations such as given by (4.7) and (4.11) at the critical point. Alternatively the expressions given by (4.14) and (4.17) may be regarded 
as determined by the integrability condition $\left[\Delta_{\sigma}+\Delta_{\sigma}^{\prime}, \Delta_{\sigma^{\prime}}+\Delta_{\sigma^{\prime}}^{\prime}\right] W=0$, without any commitment to a specific regularisation scheme [18]. Such conditions also require the absence of a $R^{2}$ contribution in addition to the $F, G$ curvature terms in (4.14). From (4.12) and (4.16) we may find to one loop

$$
\hat{\theta}=1-\frac{1}{2} \varepsilon+\frac{2}{9}(n+2) u_{*}, \quad \iota_{c}=-\frac{2}{3} \frac{n}{16 \pi^{2}}, \quad \iota_{m}=\frac{2}{3} \frac{n}{16 \pi^{2}},
$$

while from $(4.8 \mathrm{a}, \mathrm{b})$ to two loops

$$
\hat{\omega}=-\frac{1}{2} \frac{n}{16 \pi^{2}}\left(1+\frac{1}{6}(n+2) u_{*}\right) .
$$

In terms of the definition of $\beta_{c}$ in (3.20) then (4.14) requires that at the critical point $(d-1) \hat{\rho}=-\eta_{c} \hat{\theta}$ which along with (4.18) may be verified with the results (3.22) and $(4.19,20)$ using $\gamma_{m *}=\frac{1}{3}(n+2) u_{*}+\mathrm{O}\left(u_{*}^{2}\right)$. Even at the critical point $W$ is arbitrary up to the addition of local functionals of $m^{2}$ and $c$. For

$$
\delta W=\mu^{-\varepsilon} \int_{\partial \mathcal{M}} d S\left(r K^{\prime} m^{2}+s \partial_{n} m^{2}\right)-\mu^{-\varepsilon} \int_{\mathcal{M}} d v t R^{\prime} m^{2}
$$

then

$$
\delta \iota_{m}=r+\left(2-\gamma_{m *}\right) s, \quad \delta \hat{\omega}=\left(\gamma_{m *}-\varepsilon\right) s, \quad \delta k=2 t,
$$

so that in the $\varepsilon$ expansion it is possible to set $\iota_{m}$ and $k$ to zero but not $\hat{\omega}$.

Given the form of the renormalisation group equation $(4.14,15)$ at the critical point it is straightforward to see that it may be rewritten as

$$
\Delta_{\sigma} W\left(m^{2}+\frac{1}{2} \tau R^{\prime}, c+\hat{\theta} K^{\prime}, J, \hat{h}\right)=X\left(m^{2}, c\right) .
$$

Manifestly $W\left(\frac{1}{2} \tau R^{\prime}, \hat{\theta} K^{\prime}, 0,0\right)$ is invariant under local Weyl transformations, apart from the contributions involving $X$ on the r.h.s. of (4.21). This is a consequence of the result that, at least for simple field theories, invariance with respect to constant scale transformations leads to symmetry under the full conformal group [19]. Using previous results [18] in the $\varepsilon$ expansion $\tau=1-\frac{1}{2} \varepsilon+\frac{1}{54}(n+2) u_{*}^{3}+\mathrm{O}\left(\varepsilon^{4}\right)$ so there is a modification of the usual coefficient of the $\phi^{2} R$ term in a free scalar theory for conformal invariance in the interacting case at three loops.

From (4.21) it is straightforward to determine the behaviour of the composite operator $\phi^{2}$ in the neighbourhood of the boundary at the critical point. Defining

$$
\mu^{-\varepsilon}\left\langle\phi^{2}(x)\right\rangle=-\left.\frac{\delta}{\delta m^{2}(x)} W\left(m^{2}+\frac{1}{2} \tau R^{\prime}, \hat{\theta} K^{\prime}, 0,0\right)\right|_{m^{2}=0},
$$

then from (4.21) we may obtain, with the standard coordinates $x=(\mathbf{x}, y)$,

$$
\begin{aligned}
\left(\int_{\mathcal{M}} d v \sigma 2 g^{\mu \nu} \frac{\delta}{\delta g^{\mu \nu}}\right. & \left.-x_{m} \sigma(\mathbf{x}, y)\right)\left\langle\phi^{2}(\mathbf{x}, y)\right\rangle \\
= & -\hat{\omega} \sigma^{0}(\mathbf{x})\left(\delta^{\prime}(y)-\left(x_{m}-1\right) K^{\prime}(\mathbf{x}) \delta(y)\right) \\
& -\iota_{m}\left(\partial_{n} \sigma(\mathbf{x})-\left(x_{m}-2\right) \sigma^{0}(\mathbf{x}) K^{\prime}(\mathbf{x})\right) \delta(y)
\end{aligned}
$$


where $x_{m}=d-2+\gamma_{m *}$ and $\sigma^{0}(\mathbf{x})=\sigma(\mathbf{x}, 0), \partial_{n} \sigma(\mathbf{x})=\left.\partial_{y} \sigma(\mathbf{x}, y)\right|_{y=0}$. Using that under a Weyl rescaling as in (4.1)

$$
\delta_{\sigma} y=-\sigma^{0}(\mathbf{x}) y-\frac{1}{2} \partial_{n} \sigma(\mathbf{x}) y^{2}-\frac{1}{6} \nabla_{n}^{2} \sigma(\mathbf{x}) y^{3}+\ldots
$$

then we may solve (4.23) for the leading singular behaviour as $y \rightarrow 0$,

$$
\begin{aligned}
\left\langle\phi^{2}(\mathbf{x}, y)\right\rangle \sim & \left(y^{-x_{m}}+\frac{1}{2} x_{m} K^{\prime}(\mathbf{x}) y^{-x_{m}+1}\right) C \\
& -\frac{\hat{\omega}}{2-x_{m}} \delta^{\prime}(y)-\left(\iota_{m}+\frac{1-x_{m}}{2-x_{m}} \hat{\omega}\right) K^{\prime}(\mathbf{x}) \delta(y) .
\end{aligned}
$$

For $y>0$ this is in agreement with a general formula of Cardy [9], apart from a sign. The $\delta^{\prime}(y), \delta(y)$ terms reflect the regularisation of the singularity for $x_{m} \rightarrow 2$, when it is necessary that $C \rightarrow-\hat{\omega}$.

A similar discussion to the above case can also be essentially carried out in the Dirichlet case, corresponding to the calculations of section 2, with similar consequences but it is not presented because of some technical problems. 


\section{Schrödinger Equation}

The results of section 2 verify the feasibility of defining in perturbation theory a finite wave functional $\hat{\Psi}(\hat{\varphi})$ for renormalisable scalar quantum field theories on a manifold $\mathcal{M}$ where the quantum fields $\phi$ are specified by an arbitrary smooth field $\hat{\varphi}$ on the boundary $\partial \mathcal{M}$. For such wave functionals there is a corresponding Schrödinger equation which describes the variation of $\hat{\Psi}$ under smooth local deformations of $\partial \mathcal{M}$. A discussion related to that presented here was also given by Symanzik [10] although only constant global shifts of the boundary were considered. The relevant Hamiltonian is a functional differential operator requiring careful regularisation as is discussed subsequently.

The wave functional $\hat{\Psi}$ is defined formally by

$$
\hat{\Psi}(\hat{\varphi})=\int_{\left.\phi\right|_{\partial \mathcal{M}}=\hat{\varphi}} d[\phi] e^{-\frac{1}{\hbar} S_{0}(\phi)}
$$

where we have introduced Planck's constant $\hbar$ in order to set up a semiclassical expansion in powers of $\hbar$. In general the boundary of $\mathcal{M}$ is supposed to be determined by $x^{\mu}(\hat{\mathbf{x}})$ for $\hat{\mathbf{x}}$ coordinates on $\partial \mathcal{M}$ and we then consider variations along the normal to $\partial \mathcal{M}$ by taking

$$
\delta x^{\mu}(\hat{\mathbf{x}})=-n^{\mu}(\hat{\mathbf{x}}) \delta t(\hat{\mathbf{x}})
$$

The implications of such variations on the Green functions $G_{\Delta}$ defined for operators of the form (1.2) with Dirichlet boundary conditions on $\partial \mathcal{M}$ and other quantities, such as the extrinsic curvature $K_{i j}$, whose definition also depends on the specification of $\partial \mathcal{M}$ is considered in appendix C. The functional Schrödinger equation for $\hat{\Psi}$ has the general form

$$
\left(\hbar \frac{\delta}{\delta t(\hat{\mathbf{x}})}+\mathcal{H}_{0}(\hat{\mathbf{x}})\right) \hat{\Psi}(\hat{\varphi})=0, \quad \frac{\delta}{\delta t(\hat{\mathbf{x}})} \equiv-n^{\mu}(\hat{\mathbf{x}}) \frac{\delta}{\delta x^{\mu}(\hat{\mathbf{x}})},
$$

where $\mathcal{H}_{0}$ is the corresponding Hamiltonian operator. At lowest order in the loop expansion, as shown subsequently, it is sufficient to let

$$
\mathcal{H}_{0} \rightarrow \mathcal{H}=-\frac{1}{2} \mu^{\varepsilon} \hbar^{2} \frac{\delta^{2}}{\delta \hat{\varphi}^{2}}+\mu^{-\varepsilon} \mathcal{V}(\hat{\varphi})
$$

where $\mathcal{V}$ is a potential depending locally on $\hat{\varphi}$ and its derivatives in $\partial \mathcal{M}$. Although $\hat{\Psi}$ is finite for arbitrary $\hat{\varphi}$ and smooth boundary surfaces $\partial \mathcal{M}$ it is necessary to take account also of additional divergences arising from the second order functional derivative in $\mathcal{H}$, in general $\delta^{2} / \delta \hat{\varphi}(\hat{\mathbf{x}}) \delta \hat{\varphi}\left(\hat{\mathbf{x}}^{\prime}\right)$ is singular as $\hat{\mathbf{x}}^{\prime} \rightarrow \hat{\mathbf{x}}$. In our calculations using dimensional regularisation is also sufficient to ensure a well defined Hamiltonian operator $\mathcal{H}_{0}$ may be found, satisfying (5.3), which differs from $\mathcal{H}$ in (5.4) by local counterterms which are just poles in $\varepsilon$. These additional counterterms have essentially a similar form to the terms already present in $\mathcal{H}$. As shown later the detailed structure of $\mathcal{H}_{0}$ is strongly constrained by the renormalisation group equations for $\hat{\Psi}$ expressing independence of the arbitrary scale $\mu$. 
The semiclassical expansion to the wave functional $\hat{\Psi}(\hat{\varphi})$ is derived by a loop expansion of the functional integral (5.1) where the field $\phi$ is also expanded about a background classical solution $\varphi_{\mathrm{c}}$, with boundary conditions $\left.\varphi_{\mathrm{c}}\right|_{\partial \mathcal{M}}=\hat{\varphi}$. If the classical action $S_{\mathrm{c}}(\hat{\varphi})=S\left(\varphi_{\mathrm{c}}, g\right)$, since the boundary term $\hat{S}$ in $(2.1)$ vanishes for $\varphi_{\mathrm{c}}$, then to one loop order the semiclassical approximation to $\hat{\Psi}$ is, from $(2.10)$ with $\hat{\Psi}(\hat{\varphi})=\exp \Gamma\left(\hat{\varphi} ; \varphi_{\mathrm{c}}\right)$, given by

$$
\hat{\Psi}_{\text {s.c. }}(\hat{\varphi})=\left(\operatorname{det} \Delta_{\text {reg }}\right)^{-\frac{1}{2}} e^{-\frac{1}{\hbar} \mu^{-\varepsilon} S_{\mathrm{c}}(\hat{\varphi})},
$$

where $\varphi_{\mathrm{c}}$ is the classical solution, with boundary value $\hat{\varphi}$, and $\operatorname{det} \Delta_{\text {reg }}$ is the dimensional regularised functional determinant for Dirichlet boundary conditions defined as in (C.18). In order to discuss the Schrödinger equation (5.3) it is necessary to determine the effect on the classical solution $\varphi_{\mathrm{c}}$ of independent variations in the boundary value $\hat{\varphi}$ and also of deformations of $\partial \mathcal{M}$ as in (5.2). In both cases the variation $\delta \varphi_{\mathrm{c}}$ satisfies $\Delta \delta \varphi_{\mathrm{c}}=0$. For changes in $\hat{\varphi}$ then the boundary condition is simply $\left.\delta \varphi_{c}\right|_{\partial \mathcal{M}}=\delta \hat{\varphi}$ and hence

$$
\frac{\delta \varphi_{c i}(x)}{\delta \hat{\varphi}_{j}(\hat{\mathbf{x}})}=\left.G_{i j}\left(x, x^{\prime}\right) \overleftarrow{\partial}_{n}^{\prime}\right|_{x^{\prime}=x(\hat{\mathbf{x}})}
$$

with $G_{i j}\left(x, x^{\prime}\right)$ the Dirichlet Green function for $\Delta$. For changes in the boundary $\partial \mathcal{M}$, as represented by $(5.2)$, then we require

$$
\left.\left(\varphi_{\mathrm{c}}+\delta \varphi_{\mathrm{c}}\right)\right|_{x=x(\hat{\mathbf{x}})+\delta x(\hat{\mathbf{x}})}=\left.\hat{\varphi}(\hat{\mathbf{x}}) \Rightarrow \delta \varphi_{\mathrm{c}}(x)\right|_{x=x(\hat{\mathbf{x}})}=\delta t(\hat{\mathbf{x}}) \partial_{n} \varphi_{\mathrm{c}}(\hat{\mathbf{x}})
$$

and thus

$$
\frac{\delta \varphi_{\mathrm{c} i}(x)}{\delta t(\hat{\mathbf{x}})}=\left.G_{i j}\left(x, x^{\prime}\right) \overleftarrow{\partial}_{n}^{\prime}\right|_{x^{\prime}=x(\hat{\mathbf{x}})} \partial_{n} \varphi_{\mathrm{c} j}(\hat{\mathbf{x}})
$$

From (5.7) and (5.8) it is easy to see that

$$
\left(\frac{\delta}{\delta t}-\partial_{n} \varphi_{\mathrm{c} i} \frac{\delta}{\delta \hat{\varphi}_{i}}\right) \varphi_{\mathrm{c}}(x)=0
$$

which is important in later calculations.

The verification that the semiclassical form for the wave function in (5.5) is an approximate solution of the Schrödinger equation, which becomes exact for quadratic Hamiltonians, is well known in conventional quantum mechanics [20]. Here we extend the analysis to the next leading, or two loop, approximation which is straightforward but also pay special attention to the divergences which arise in a field theory context. To start it is necessary to consider how the classical action $S_{\mathrm{c}}$ depends on $\hat{\varphi}$ and also its dependence on changes in $\partial \mathcal{M}$ as given by (5.2). By integration by parts and using the classical equation for $\varphi_{\mathrm{c}}$, derived by requiring $S_{\mathrm{c}}$ to be stationary at $\phi=\hat{\varphi}$,

$$
S_{\mathrm{c}}(\hat{\varphi})=\int_{\mathcal{M}} d v\left(V\left(\varphi_{\mathrm{c}}\right)-\frac{1}{2} V_{i}^{\prime}\left(\varphi_{\mathrm{c}}\right) \varphi_{\mathrm{c} i}\right)-\frac{1}{2} \int_{\partial \mathcal{M}} d S \hat{\varphi}_{i} \partial_{n} \varphi_{\mathrm{c} i}
$$

With this result then under arbitrary variations in $\hat{\varphi}$ and also $\varphi_{\mathrm{c}}$, subject to $\Delta \delta \varphi_{\mathrm{c}}=0$, we find

$$
\delta S_{\mathrm{c}}(\hat{\varphi})=-\frac{1}{2} \int_{\partial \mathcal{M}} d S\left(\delta \varphi_{\mathrm{c} i}+\delta \hat{\varphi}_{i}\right) \partial_{n} \varphi_{\mathrm{c} i}
$$


Hence, taking $\delta \varphi_{\mathrm{c}}=\delta \hat{\varphi}$,

$$
\frac{\delta}{\delta \hat{\varphi}_{i}(\hat{\mathbf{x}})} S_{\mathrm{c}}(\hat{\varphi})=-\partial_{n} \varphi_{\mathrm{c} i}(\hat{\mathbf{x}})
$$

while, using (5.7) and also (C.8a,b) with (C.5) and the equation of motion for $\varphi_{\mathrm{c}}$ on the boundary, we derive a local version of the Hamilton-Jacobi equation

$$
\frac{\delta}{\delta t(\hat{\mathbf{x}})} S_{\mathrm{c}}(\hat{\varphi})=-\frac{1}{2} \partial_{n} \varphi_{\mathrm{c} i}(\hat{\mathbf{x}}) \partial_{n} \varphi_{\mathrm{c} i}(\hat{\mathbf{x}})+V(\hat{\varphi}(\hat{\mathbf{x}}))+\frac{1}{2} \hat{\gamma}^{p q} \partial_{p} \hat{\varphi}_{i}(\hat{\mathbf{x}}) \partial_{q} \hat{\varphi}_{i}(\hat{\mathbf{x}})
$$

The essential results $(5.10,11)$ ensure that at lowest order in $(5.3)$ it is sufficient to take for $\mathcal{V}$ in $(5.4)$

$$
\mathcal{V}(\hat{\varphi})=V(\hat{\varphi})+\frac{1}{2} \hat{\gamma}^{p q} \partial_{p} \hat{\varphi}_{i} \partial_{q} \hat{\varphi}_{i}
$$

since then

$$
\left(\hbar \frac{\delta}{\delta t}+\mathcal{H}\right) e^{-\frac{1}{\hbar} \mu^{-\varepsilon} S_{\mathrm{c}}(\hat{\varphi})}=\frac{1}{2} \hbar \frac{\delta^{2}}{\delta \hat{\varphi}^{2}} S_{\mathrm{c}}(\hat{\varphi}) e^{-\frac{1}{\hbar} \mu^{-\varepsilon} S_{\mathrm{c}}(\hat{\varphi})}, \frac{\delta^{2}}{\delta \hat{\varphi}(\hat{\mathbf{x}})^{2}} S_{\mathrm{c}}(\hat{\varphi})=-\mathcal{K}_{i i}(\hat{\mathbf{x}}, \hat{\mathbf{x}})
$$

from (5.6) and (5.10) where $\mathcal{K}$ is defined as in (C.22),

$$
\mathcal{K}_{i j}\left(\hat{\mathbf{x}}, \hat{\mathbf{x}}^{\prime}\right)=\left.\partial_{n} G_{i j}\left(x, x^{\prime}\right) \overleftarrow{\partial}_{n}^{\prime}\right|_{x=x(\hat{\mathbf{x}}), x^{\prime}=x\left(\hat{\mathbf{x}}^{\prime}\right)}
$$

From (5.9) and (C.21) we may find

$$
\left(\frac{\delta}{\delta t}-\partial_{n} \varphi_{\mathrm{c} i} \frac{\delta}{\delta \hat{\varphi}_{i}}\right) \ln \operatorname{det} \Delta_{\mathrm{reg}}=\frac{\delta^{2}}{\delta \hat{\varphi}^{2}} S_{\mathrm{c}}(\hat{\varphi})+\frac{2 \mu^{-\varepsilon}}{16 \pi^{2} \varepsilon} \operatorname{tr}\left(\hat{\mathcal{B}}_{4}\right)
$$

where the pole term in $\varepsilon$ is necessary for the subtraction of divergences arising from $\delta^{2} / \delta \hat{\varphi}^{2}$ in this case. Explicitly from (C.17), considering only terms which depend on $V$ and are at most linear in $K$, and using the equation of motion on $\partial \mathcal{M} \nabla_{n}^{2} \varphi_{\mathrm{c}}-K \partial_{n} \varphi_{\mathrm{c}}+\hat{\nabla}^{2} \hat{\varphi}=V^{\prime}(\hat{\varphi})$,

$$
\begin{aligned}
\hat{\mathcal{B}}_{4 i j}= & \frac{1}{2} V_{i k}^{\prime \prime} V_{k j}^{\prime \prime}-\frac{1}{2} \nabla_{n}^{2} V_{i j}^{\prime \prime}-\frac{1}{6} \hat{\nabla}^{2} V_{i j}^{\prime \prime}+\frac{5}{6} K \partial_{n} V_{i j}^{\prime \prime}+\ldots, \\
\operatorname{tr}\left(\hat{\mathcal{B}}_{4}\right)= & \frac{1}{2} V_{i j}^{\prime \prime}(\hat{\varphi}) V_{i j}^{\prime \prime}(\hat{\varphi})-\frac{1}{2} V_{i i j}^{\prime \prime \prime}(\hat{\varphi}) V_{j}^{\prime}(\hat{\varphi})-\frac{1}{2} g_{i j k k} \partial_{n} \varphi_{\mathrm{c} i} \partial_{n} \varphi_{\mathrm{c} j} \\
& -\frac{1}{6} g_{i j k k} \hat{\gamma}^{p q} \partial_{p} \hat{\varphi}_{i} \partial_{q} \hat{\varphi}_{i}+\frac{1}{3} V_{i i j}^{\prime \prime \prime}(\hat{\varphi}) \hat{\nabla}^{2} \hat{\varphi}_{j}+\frac{1}{3} K V_{i i j}^{\prime \prime \prime}(\hat{\varphi}) \partial_{n} \varphi_{\mathrm{c} j}
\end{aligned}
$$

which is a local function of $\hat{\varphi}$ and $\partial_{n} \varphi_{\mathrm{c}}$ on $\partial \mathcal{M}$. In consequence from (5.13) and (5.15)

$$
\begin{gathered}
\left(\hbar \frac{\delta}{\delta t}+\mathcal{H}\right) \hat{\Psi}_{\text {s.c. }}(\hat{\varphi})=-\frac{\hbar \mu^{-\varepsilon}}{16 \pi^{2} \varepsilon} \operatorname{tr}\left(\hat{\mathcal{B}}_{4}\right) \hat{\Psi}_{\text {s.c. }}(\hat{\varphi})+\hbar^{2} \mathcal{X}_{1} \hat{\Psi}_{\text {s.c. }}(\hat{\varphi}) \\
\mathcal{X}_{1}=\frac{1}{4} \mu^{\varepsilon}\left(\frac{\delta^{2}}{\delta \hat{\varphi}^{2}} \ln \operatorname{det} \Delta_{\text {reg }}-\frac{1}{2} \frac{\delta}{\delta \hat{\varphi}_{i}} \ln \operatorname{det} \Delta_{\text {reg }} \frac{\delta}{\delta \hat{\varphi}_{i}} \ln \operatorname{det} \Delta_{\text {reg }}\right)
\end{gathered}
$$


To first order in $\hbar \mathcal{X}_{1}$ can be neglected and so $\hat{\Psi}_{\text {s.c. }}$ satisfies the Schrödinger equation (5.3) in the leading semiclassical approximation if

$$
\begin{aligned}
\mathcal{H}_{0}^{(1)} & =\frac{\hbar}{16 \pi^{2} \varepsilon}\left(-\frac{1}{2} \mu^{\varepsilon} \hbar^{2} g_{i j k k} \frac{\delta^{2}}{\delta \hat{\varphi}_{i} \delta \hat{\varphi}_{j}}+\frac{1}{3} \hbar K V_{i i j}^{\prime \prime \prime}(\hat{\varphi}) \frac{\delta}{\delta \hat{\varphi}_{j}}\right)+\mu^{-\varepsilon} \mathcal{V}_{0}^{(1)}(\hat{\varphi}), \\
\mathcal{V}_{0}^{(1)}(\hat{\varphi}) & =\frac{\hbar}{16 \pi^{2} \varepsilon}\left(\frac{1}{2} V_{i j}^{\prime \prime}(\hat{\varphi}) V_{i j}^{\prime \prime}(\hat{\varphi})-\frac{1}{2} V_{i i j}^{\prime \prime \prime}(\hat{\varphi}) V_{j}^{\prime}(\hat{\varphi})-\frac{1}{2} g_{i j k k} \hat{\gamma}^{p q} \partial_{p} \hat{\varphi}_{i} \partial_{q} \hat{\varphi}_{i}+\frac{1}{3} \hat{\nabla}^{2} V_{i i}^{\prime \prime \prime}(\hat{\varphi})\right)
\end{aligned}
$$

since, with these expressions, it is easy to see that

$$
\begin{aligned}
\mathcal{H}_{0}^{(1)} \hat{\Psi}_{\text {s.c. }}(\hat{\varphi}) & =\frac{\hbar \mu^{-\varepsilon}}{16 \pi^{2} \varepsilon} \operatorname{tr}\left(\hat{\mathcal{B}}_{4}\right) \hat{\Psi}_{\text {s.c. }}(\hat{\varphi})+\left(\hbar^{2}\left(\mathcal{X}_{2}+\mathcal{X}_{3}\right)+\mathrm{O}\left(\hbar^{3}\right)\right) \hat{\Psi}_{\text {s.c. }}(\hat{\varphi}) \\
\mathcal{X}_{2} & =\frac{1}{2} \frac{1}{16 \pi^{2} \varepsilon}\left(g_{i j k k} \partial_{n} \varphi_{\mathrm{c} i}-\frac{1}{3} K V_{i i j}^{\prime \prime \prime}(\hat{\varphi})\right) \frac{\delta}{\delta \hat{\varphi}_{j}} \ln \operatorname{det} \Delta_{\mathrm{reg}}, \\
\mathcal{X}_{3} & =\frac{1}{2} \frac{1}{16 \pi^{2} \varepsilon} g_{i j k k} \frac{\delta^{2}}{\delta \hat{\varphi}_{i} \delta \hat{\varphi}_{j}} S_{\mathrm{c}}(\hat{\varphi}), \quad \frac{\delta^{2}}{\delta \hat{\varphi}_{i}(\hat{\mathbf{x}}) \delta \hat{\varphi}_{j}(\hat{\mathbf{x}})} S_{\mathrm{c}}(\hat{\varphi})=-\mathcal{K}_{i j}(\hat{\mathbf{x}}, \hat{\mathbf{x}}) .
\end{aligned}
$$

At the next order in the semiclassical expansion of the wave functional we may write

$$
\hat{\Psi}(\hat{\varphi})=\hat{\Psi}_{\text {s.c. }}(\hat{\varphi}) e^{\hbar \Gamma_{\text {reg }}^{(2)}+\mathrm{O}\left(\hbar^{2}\right)}, \quad \Gamma_{\text {reg }}^{(2)}=\Gamma^{(2)}-S_{0}^{(2)},
$$

where $\Gamma^{(2)}=\Gamma_{a}+\Gamma_{b}+\Gamma_{c}+\Gamma_{d}$ as given in (2.13), (2.15), (2.17) and (2.18) while $S_{0}^{(2)}\left(\varphi_{\mathrm{c}}\right)$ is the overall local two loop counterterm which subtracts the divergent poles in $\varepsilon$. The calculations of section 2 determine the specific boundary terms. From $(2.7,9)$ and using the equations of motion for $\varphi_{\mathrm{c}}$ this may be expressed in the convenient form

$$
S_{0}^{(2)}\left(\varphi_{\mathrm{c}}\right)=\int_{\mathcal{M}} d v V_{0}^{(2)}\left(\mu^{-\frac{1}{2} \varepsilon} \varphi_{\mathrm{c}}\right)+\int_{\partial \mathcal{M}} d S\left(-\mu^{-\frac{1}{2} \varepsilon} \hat{\varphi}_{0 i}^{(2)}(\hat{\varphi}) \partial_{n} \varphi_{\mathrm{c} i}+\mu^{-\varepsilon} \rho^{(2)}(\hat{\varphi}) K\right)+\ldots
$$

where $V_{0}\left(\phi_{0}\right)=\mu^{-\varepsilon}\left(V(\phi)+V_{\text {c.t. }}(\phi)\right)$ and $\hat{\varphi}_{0}^{(2)}$ is given in terms of $Z^{(2)}$ and $\hat{\varphi}_{\text {c.t. }}^{(2)}$ by $(2.25)$. In order to evaluate $\mathcal{X}_{1}, \mathcal{X}_{2}$ in $(5.16,18)$ we need to obtain an analogous formula to (C.19) expressing the dependence of det $\Delta_{\text {reg }}$ on $\hat{\varphi}$. Using (5.6) and also the explicit form of the counterterms given by (1.17) in this case

$$
\begin{aligned}
\frac{\delta}{\delta \hat{\varphi}_{i}(\hat{\mathbf{x}})} \ln \operatorname{det} \Delta_{\mathrm{reg}} & =\int d v \bar{G}_{m n}\left|(x) V_{m n j}^{\prime \prime \prime}\left(\varphi_{\mathrm{c}}\right) G_{j i}(x, \hat{x}) \overleftarrow{\partial}_{n}\right|_{\hat{x}=x(\hat{\mathbf{x}})} \\
+ & \frac{\mu^{-\varepsilon}}{16 \pi^{2} \varepsilon}\left\{\int d S^{\prime} V_{k^{\prime} k^{\prime} j^{\prime}}^{\prime \prime \prime}\left(\hat{\varphi}^{\prime}\right) \mathcal{K}_{j^{\prime} i}\left(\hat{\mathbf{x}}^{\prime}, \hat{\mathbf{x}}\right)+g_{k k i j} \partial_{n} \varphi_{\mathrm{c} j}(\hat{\mathbf{x}})-\frac{2}{3} K(\hat{\mathbf{x}}) V_{j j i}^{\prime \prime \prime}(\hat{\varphi})\right\}
\end{aligned}
$$

where $\bar{G}_{m n} \mid$ is defined by $(2.14)$ and $\mathcal{K}$ is as in (5.14). Furthermore since $\delta G=-G \delta \Delta G$

$$
\frac{\delta}{\delta \hat{\varphi}_{i}(\hat{\mathbf{x}})} G_{j_{1} j_{2}}\left(x_{1}, x_{2}\right)=-\left.\int d v G_{j_{1} j}\left(x_{1}, x\right) V_{j k \ell}^{\prime \prime \prime}\left(\varphi_{c}\right) G_{k j_{2}}\left(x, x_{2}\right) G_{\ell i}(x, \hat{x}) \overleftarrow{\partial}_{n}\right|_{\hat{x}=x(\hat{\mathbf{x}})}
$$


we may also obtain in turn from (5.22) (as usual with dimensional regularisation we set $\delta^{d-1}(0)=0$ so that the $K$ term in (5.22) does not contribute to the second functional derivative here)

$$
\begin{aligned}
\frac{\delta^{2}}{\delta \hat{\varphi}(\hat{\mathbf{x}})^{2}} & \ln \operatorname{det} \Delta_{\mathrm{reg}} \\
= & \int d v \bar{G}_{m n}\left|(x) g_{m n i j} G_{j k}(x, \hat{x}) \overleftarrow{\partial}_{n} \partial_{n} G_{k i}(\hat{x}, x)\right|_{\hat{x}=x(\hat{\mathbf{x}})} \\
& -\int d v d v^{\prime} \bar{G}_{m n}\left|(x) V_{m n j}^{\prime \prime \prime}\left(\varphi_{\mathrm{c}}\right) G_{j j^{\prime}}\left(x, x^{\prime}\right) V_{j^{\prime} k^{\prime} \ell^{\prime}}^{\prime \prime \prime}\left(\varphi_{\mathrm{c}}^{\prime}\right) G_{k^{\prime} i}\left(x^{\prime}, \hat{x}\right) \overleftarrow{\partial_{n}} \partial_{n} G_{i \ell^{\prime}}\left(\hat{x}, x^{\prime}\right)\right|_{\hat{x}=x(\hat{\mathbf{x}})} \\
& -\int d v d v^{\prime} \partial_{n} G_{i j}(\hat{x}, x) V_{j m n}^{\prime \prime \prime}\left(\varphi_{\mathrm{c}}\right) \\
& \quad \times\left. G_{m m^{\prime}}\left(x, x^{\prime}\right) G_{n n^{\prime}}\left(x, x^{\prime}\right) V_{m^{\prime} n^{\prime} j^{\prime}}^{\prime \prime \prime}\left(\varphi_{\mathrm{c}}^{\prime}\right) G_{j^{\prime} i}\left(x^{\prime}, \hat{x}\right) \overleftarrow{\partial_{n}}\right|_{\hat{x}=x(\hat{\mathbf{x}})} \\
& -\left.\left.\frac{2 \mu^{-\varepsilon}}{16 \pi^{2} \varepsilon} \int d v \partial_{n} G_{i j}(\hat{x}, x) V_{j m n}^{\prime \prime \prime}\left(\varphi_{\mathrm{c}}\right) V_{m n k}^{\prime \prime \prime}\left(\varphi_{\mathrm{c}}\right) G_{k i}(x, \hat{x}) \overleftarrow{\partial_{n}}\right|_{\hat{x}=x(\hat{\mathbf{x}})} \int d S^{\prime} d v V_{k^{\prime} k^{\prime} j^{\prime}}^{\prime \prime}\left(\hat{\varphi}^{\prime}\right) \partial_{n}^{\prime} G_{j^{\prime} k}\left(x^{\prime}, x\right) V_{k \ell m}^{\prime \prime \prime}\left(\varphi_{\mathrm{c}}\right) G_{m i}(x, \hat{x}) \overleftarrow{\partial_{n}} \partial_{n} G_{i \ell}(\hat{x}, x)\right|_{\hat{x}=x(\hat{\mathbf{x}}), x^{\prime}=x\left(\hat{\mathbf{x}}^{\prime}\right)} \\
& +\frac{2 \mu^{-\varepsilon}}{16 \pi^{2} \varepsilon} g_{k k i j} \mathcal{K}_{j i}(\hat{\mathbf{x}}, \hat{\mathbf{x}}) .
\end{aligned}
$$

In order to verify that (5.20) is a valid approximation to two loop order it is sufficient to show

$$
\left(\frac{\delta}{\delta t}-\partial_{n} \varphi_{\mathrm{c} i} \frac{\delta}{\delta \hat{\varphi}_{i}}\right) \Gamma_{\mathrm{reg}}^{(2)}=-\mathcal{X}_{1}-\mathcal{X}_{2}-\mathcal{X}_{\mathrm{loc}}
$$

since this entails

$$
\left(\hbar \frac{\delta}{\delta t}+\mathcal{H}+\mathcal{H}_{0}^{(1)}\right) \hat{\Psi}(\hat{\varphi})=-\left(\hbar^{2} \mathcal{X}_{\mathrm{loc}}+\mathrm{O}\left(\hbar^{3}\right)\right) \hat{\Psi}(\hat{\varphi})
$$

After using the equation of motion for $\varphi_{\mathrm{c}}$ to eliminate $\nabla_{n}^{2} \varphi_{\mathrm{c}}$ we assume that $\mathcal{X}_{\mathrm{loc}}\left(\hat{\varphi}, \partial_{n} \varphi_{\mathrm{c}}\right)$ is local function, which for the renormalisable theory considered here is of dimension 4 . This may then be cancelled by an appropriate choice for

$$
\mathcal{H}_{0}^{(2)}=\hbar^{2} \mathcal{X}_{\mathrm{loc}}(\hat{\varphi}, \hat{p}), \quad \hat{p}=\mu^{\varepsilon} \hbar \frac{\delta}{\delta \hat{\varphi}},
$$

by virtue of (5.10). We therefore endeavour to demonstrate (5.24) by considering each contribution to $\Gamma^{(2)}$ in turn, with $\varphi \rightarrow \varphi_{\mathrm{c}}$, and using (5.9) with (C.8a,b), (C.11). The surface terms arising according to (C.8a) from the differentiation of the volume integrals expressing $\Gamma_{a}, \Gamma_{b}, \Gamma_{c}$ vanish since $G$ satisfies Dirichlet boundary conditions. Beginning with the expression for $\Gamma_{a}$ in (2.13) it is simple to obtain

$$
\begin{aligned}
\left(\frac{\delta}{\delta t(\hat{\mathbf{x}})}\right. & \left.-\partial_{n} \varphi_{\mathrm{c} i}(\hat{\mathbf{x}}) \frac{\delta}{\delta \hat{\varphi}_{i}(\hat{\mathbf{x}})}\right) \Gamma_{a} \\
& =-\frac{\mu^{\varepsilon}}{4} \int d v g_{i j k \ell} \bar{G}_{i j}\left|(x) G_{k m}(x, \hat{x}) \overleftarrow{\partial_{n}} \partial_{n} G_{m \ell}(\hat{x}, x)\right|_{\hat{x}=x(\hat{\mathbf{x}})}
\end{aligned}
$$


For $\Gamma_{b}$ given by (2.15) the corresponding result is

$$
\begin{aligned}
& \left(\frac{\delta}{\delta t(\hat{\mathbf{x}})}-\partial_{n} \varphi_{\mathrm{c} i}(\hat{\mathbf{x}}) \frac{\delta}{\delta \hat{\varphi}_{i}(\hat{\mathbf{x}})}\right) \Gamma_{b} \\
& =\frac{\mu^{\varepsilon}}{4} \iint d v d v^{\prime} V_{i j k}^{\prime \prime \prime}\left(\varphi_{\mathrm{c}}\right) G_{i i^{\prime}}\left(x, x^{\prime}\right) G_{j j^{\prime}}\left(x, x^{\prime}\right) \\
& \quad \times\left. G_{k \ell}(x, \hat{x}) \overleftarrow{\partial_{n}} \partial_{n} G_{\ell k^{\prime}}\left(\hat{x}, x^{\prime}\right) V_{i^{\prime} j^{\prime} k^{\prime}}^{\prime \prime \prime}\left(\varphi_{\mathrm{c}}^{\prime}\right)\right|_{\hat{x}=x(\hat{\mathbf{x}})} \\
& -\left.\frac{1}{2} \frac{1}{16 \pi^{2} \varepsilon} \int d v V_{i j k}^{\prime \prime \prime}\left(\varphi_{\mathrm{c}}\right) V_{i j \ell}^{\prime \prime \prime}\left(\varphi_{\mathrm{c}}\right) G_{k m}(x, \hat{x}) \overleftarrow{\partial}_{n} \partial_{n} G_{m \ell}(\hat{x}, x)\right|_{\hat{x}=x(\hat{\mathbf{x}})}
\end{aligned}
$$

For the one particle reducible amplitude given by (2.17) we may obtain in this case

$$
\begin{aligned}
\left(\frac{\delta}{\delta t(\hat{\mathbf{x}})}\right. & \left.-\partial_{n} \varphi_{\mathrm{c} i}(\hat{\mathbf{x}}) \frac{\delta}{\delta \hat{\varphi}_{i}(\hat{\mathbf{x}})}\right) \Gamma_{c} \\
= & \left.\frac{\mu^{\varepsilon}}{8} \iint d v d v^{\prime} \bar{G}_{j k}\left|(x) V_{i j k}^{\prime \prime \prime}\left(\varphi_{\mathrm{c}}\right) G_{i \ell}(x, \hat{x}) \overleftarrow{\partial}_{n} \partial_{n} G_{\ell i^{\prime}}\left(\hat{x}, x^{\prime}\right) V_{i^{\prime} j^{\prime} k^{\prime}}^{\prime \prime \prime}\left(\varphi_{\mathrm{c}}^{\prime}\right) \bar{G}_{j^{\prime} k^{\prime}}\right|\left(x^{\prime}\right)\right|_{\hat{x}=x(\hat{\mathbf{x}})} \\
& +\frac{\mu^{\varepsilon}}{4} \iint d v d v^{\prime} \bar{G}_{j k} \mid(x) V_{i j k}^{\prime \prime \prime}\left(\varphi_{\mathrm{c}}\right) G_{i i^{\prime}}\left(x, x^{\prime}\right) \\
& \times\left. V_{i^{\prime} j^{\prime} k^{\prime}}^{\prime \prime \prime}\left(\varphi_{\mathrm{c}}^{\prime}\right) G_{j^{\prime} \ell}\left(x^{\prime}, \hat{x}\right) \overleftarrow{\partial}_{n} \partial_{n} G_{\ell k^{\prime}}\left(\hat{x}, x^{\prime}\right)\right|_{\hat{x}=x(\hat{\mathbf{x}})} .
\end{aligned}
$$

For the remaining term in (2.18), using now (C.23) and (C.24) as well as (C.11) and also $\bar{G}_{k \ell} \mid=2 \mu^{-\varepsilon} \tilde{V}_{k \ell}(\hat{\varphi}) /\left(16 \pi^{2} \varepsilon\right)$ on $\partial \mathcal{M}$.

$$
\begin{aligned}
&\left(\frac{\delta}{\delta t(\hat{\mathbf{x}})}\right.\left.-\partial_{n} \varphi_{\mathrm{c} i}(\hat{\mathbf{x}}) \frac{\delta}{\delta \hat{\varphi}_{i}(\hat{\mathbf{x}})}\right) \Gamma_{d} \\
&= \frac{1}{4} \frac{1}{16 \pi^{2} \varepsilon}\left\{-\left.g_{i j k k} \partial_{n} \varphi_{\mathrm{c} j}(\hat{\mathbf{x}}) \int d v^{\prime} \partial_{n} G_{i i^{\prime}}\left(x, x^{\prime}\right)\right|_{x=x(\hat{\mathbf{x}})} V_{i^{\prime} j^{\prime} k^{\prime}}^{\prime \prime \prime}\left(\varphi_{\mathrm{c}}^{\prime}\right) \bar{G}_{j^{\prime} k^{\prime}} \mid\left(x^{\prime}\right)\right. \\
&+\left.\int d S^{\prime \prime} d v^{\prime} V_{k \ell \ell}^{\prime \prime \prime}\left(\hat{\varphi}^{\prime \prime}\right) \mathcal{K}_{k i}\left(\hat{\mathbf{x}}^{\prime \prime \prime}, \hat{\mathbf{x}}\right) \partial_{n} G_{i i^{\prime}}\left(\hat{x}, x^{\prime}\right)\right|_{\hat{x}=x(\hat{\mathbf{x}})} V_{i^{\prime} j^{\prime} k^{\prime}}^{\prime \prime \prime}\left(\varphi_{\mathrm{c}}^{\prime}\right) \bar{G}_{j^{\prime} k^{\prime}} \mid\left(x^{\prime}\right) \\
&+\int d S^{\prime \prime} d v^{\prime} V_{j \ell \ell}^{\prime \prime \prime}\left(\hat{\varphi}^{\prime \prime}\right) \partial_{n} G_{j i^{\prime}}\left(\hat{x}^{\prime \prime}, x^{\prime}\right) V_{i^{\prime} j^{\prime} k^{\prime}}^{\prime \prime \prime}\left(\varphi_{\mathrm{c}}^{\prime}\right) \\
&\left.\times\left. G_{j^{\prime} i}\left(x^{\prime}, \hat{x}\right) \overleftarrow{\partial_{n}} \partial_{n} G_{i k^{\prime}}\left(\hat{x}, x^{\prime}\right)\right|_{\left.\hat{x}=x(\hat{\mathbf{x}}), \hat{x}^{\prime \prime}=x\left(\hat{\mathbf{x}}^{\prime \prime}\right)\right\}}\right\} \\
&+\frac{1}{8} \frac{\mu^{-\varepsilon}}{\left(16 \pi^{2} \varepsilon\right)^{2}}\left\{4 V_{i j j}^{\prime \prime \prime}(\hat{\varphi}) V_{i k \ell}^{\prime \prime \prime}(\hat{\varphi}) \tilde{V}_{k \ell}(\hat{\varphi})-2 g_{i j k k} \partial_{n} \varphi_{\mathrm{c} j}(\hat{\mathbf{x}}) \int d S^{\prime} \mathcal{K}_{i i^{\prime}}\left(\hat{\mathbf{x}}, \hat{\mathbf{x}}^{\prime}\right) V_{i^{\prime} j^{\prime} j^{\prime}}^{\prime \prime \prime}\left(\hat{\varphi}^{\prime}\right)\right. \\
&+ \int d S^{\prime} d S^{\prime \prime \prime} V_{i j j}^{\prime \prime \prime}\left(\hat{\varphi}^{\prime \prime}\right) \mathcal{K}_{i k}\left(\hat{\mathbf{x}}^{\prime \prime}, \hat{\mathbf{x}}\right) \mathcal{K}_{k i^{\prime}}\left(\hat{\mathbf{x}}, \hat{\mathbf{x}}^{\prime}\right) V_{i^{\prime} j^{\prime} j^{\prime}}^{\prime \prime \prime}\left(\hat{\varphi}^{\prime}\right) \\
&\left.-\hat{\gamma}^{p q} \partial_{p} V_{i j j}^{\prime \prime \prime}(\hat{\varphi}) \partial_{q} V_{i k k}^{\prime \prime \prime}(\hat{\varphi})-V_{i k k}^{\prime \prime \prime}(\hat{\varphi}) V_{i j}^{\prime \prime}(\hat{\varphi}) V_{j \ell \ell}^{\prime \prime \prime}(\hat{\varphi})\right\} .
\end{aligned}
$$

Combining the results for the individual contributions we therefore find

$$
\left(\frac{\delta}{\delta t}-\partial_{n} \varphi_{\mathrm{c} i} \frac{\delta}{\delta \hat{\varphi}_{i}}\right) \Gamma^{(2)}=-\mathcal{X}_{1}-\mathcal{X}_{2}-\mathcal{X}_{3}-\mathcal{X}_{\mathrm{loc}, 1}
$$


where, neglecting $K^{2}$ terms,

$$
\begin{aligned}
\mathcal{X}_{\mathrm{loc}, 1}=\frac{\mu^{-\varepsilon}}{\left(16 \pi^{2} \varepsilon\right)^{2}}\{ & -\frac{3}{8} g_{i k \ell \ell} g_{j k m m} \partial_{n} \varphi_{\mathrm{c} i} \partial_{n} \varphi_{\mathrm{c} j}+\frac{1}{3} g_{i j k k} V_{j \ell \ell}^{\prime \prime \prime}(\hat{\varphi}) K \partial_{n} \varphi_{\mathrm{c} i} \\
& -\frac{1}{2} V_{i j j}^{\prime \prime \prime}(\hat{\varphi}) V_{i k \ell}^{\prime \prime \prime}(\hat{\varphi}) \tilde{V}_{k \ell}(\hat{\varphi}) \\
& \left.+\frac{1}{8} g_{i k k m} g_{j \ell \ell m} \hat{\gamma}^{p q} \partial_{p} \hat{\varphi}_{i} \partial_{q} \hat{\varphi}_{j}+\frac{1}{8} V_{i k k}^{\prime \prime \prime}(\hat{\varphi}) V_{i j}^{\prime \prime}(\hat{\varphi}) V_{j \ell \ell}^{\prime \prime \prime}(\hat{\varphi})\right\} .
\end{aligned}
$$

This result is the critical part of verifying (5.24) but in addition we need to consider the contribution arising from the overall two loop counterterms as in (5.21)

$$
\begin{aligned}
\left(\frac{\delta}{\delta t}-\right. & \left.\partial_{n} \varphi_{\mathrm{c} i} \frac{\delta}{\delta \hat{\varphi}_{i}}\right) S_{0}^{(2)}\left(\varphi_{\mathrm{c}}\right)=\mathcal{X}_{\mathrm{loc}, 2} \\
= & \mu^{-\frac{1}{2} \varepsilon} \hat{\varphi}_{0 i, j}^{(2) \prime}(\hat{\varphi})\left(\partial_{n} \varphi_{\mathrm{c} i} \partial_{n} \varphi_{\mathrm{c} j}+\hat{\gamma}^{p q} \partial_{p} \hat{\varphi}_{i} \partial_{q} \hat{\varphi}_{j}\right)+V_{0}^{(2)}\left(\mu^{-\frac{1}{2} \varepsilon} \hat{\varphi}\right) \\
& \quad+\mu^{-\frac{1}{2} \varepsilon} V_{i}^{\prime}(\hat{\varphi}) \hat{\varphi}_{0 i}^{(2)}-\mu^{-\varepsilon}\left(\rho_{i}^{(2) \prime}(\hat{\varphi}) \partial_{n} \varphi_{\mathrm{c} i} K+\hat{\nabla}^{2} \rho^{(2)}(\hat{\varphi})\right)+\ldots .
\end{aligned}
$$

Hence (5.31) and (5.33) together ensure the validity of the crucial equations (5.24) or (5.25) with $\mathcal{X}_{\text {loc }}=\mathcal{X}_{\text {loc }, 1}+\mathcal{X}_{\text {loc }, 2}$ which may then be used to determine $\mathcal{H}_{0}^{(2)}$, as described in (5.26), so that $\hat{\Psi}(\hat{\varphi})$ satisfies (5.3) at two loop order.

Together with (5.17) the result of the calculations here are compatible with the general form

$$
\begin{aligned}
\mathcal{H}_{0}= & -\frac{1}{2} \hbar^{2} \frac{\delta^{2}}{\delta \hat{\varphi}_{0}^{2}}-\hbar K \rho_{0 i}^{\prime}\left(\hat{\varphi}_{0}\right) \frac{\delta}{\delta \hat{\varphi}_{0 i}}+\mathcal{V}_{0}\left(\hat{\varphi}_{0}\right) \\
& \mathcal{V}_{0}\left(\hat{\varphi}_{0}\right)=V_{0}\left(\hat{\varphi}_{0}\right)+\frac{1}{2} \hat{\gamma}^{p q} \partial_{p} \hat{\varphi}_{0 i} \partial_{q} \hat{\varphi}_{0 i}-\hat{\nabla}^{2} \rho_{0}\left(\hat{\varphi}_{0}\right)+\ldots
\end{aligned}
$$

for $\rho_{0}\left(\hat{\varphi}_{0}\right)=\mu^{-\varepsilon} \rho(\hat{\varphi})$ and where the neglected terms represent contributions to $\mathcal{V}_{0}$ involving scalars formed from the metric of dimension 2 or 4 . The essentially complete expression for $\mathcal{H}_{0}$ to all orders, in terms of bare quantities, shown in (5.34) is a direct result of the requirement of consistency of the Schrödinger equation (5.3) with the renormalisation group equation (2.27) for the wave functional $\hat{\Psi}$ which is here written as

$$
\mathcal{D} \hat{\Psi}(\hat{\varphi})=\frac{1}{\hbar}\left(\mathcal{C}\left(\beta^{\rho}\right)+\tilde{\mathcal{C}}\left(\beta^{\lambda}, \beta^{\hat{\lambda}}\right)\right) \hat{\Psi}(\hat{\varphi}) .
$$

Applying the renormalisation group operator $\mathcal{D}$, as given by $(2.26)$, to (5.3) then since from $(2.29 \mathrm{a}, \mathrm{b}) \mathcal{D} \hat{\varphi}_{0}=0, \mathcal{D} \rho_{0}=-\mu^{-\varepsilon} \beta^{\rho}$ we may use

$$
\left[\mathcal{D}, \frac{\delta}{\delta t}\right]=0, \quad\left[\mathcal{D}, \mathcal{H}_{0}\right]=\hbar \mu^{-\varepsilon} K \frac{\partial}{\partial \hat{\varphi}_{0 i}} \beta^{\rho}(\hat{\varphi}) \frac{\delta}{\delta \hat{\varphi}_{0 i}}+\mu^{-\varepsilon} \hat{\nabla}^{2} \beta^{\rho}(\hat{\varphi})+\ldots
$$

and also, with $\mathcal{C}$ defined by $(2.24)$,

$$
\begin{aligned}
{\left[\hbar \frac{\delta}{\delta t}, \frac{1}{\hbar} \mathcal{C}\left(\beta^{\rho}\right)\right] } & =-\mu^{-\varepsilon} \hat{\nabla}^{2} \beta^{\rho}(\hat{\varphi})+\ldots \\
{\left[\mathcal{H}_{0}, \frac{1}{\hbar} \mathcal{C}\left(\beta^{\rho}\right)\right] } & =-\hbar \mu^{-\varepsilon} K \frac{\partial}{\partial \hat{\varphi}_{0 i}} \beta^{\rho}(\hat{\varphi}) \frac{\delta}{\delta \hat{\varphi}_{0 i}}+\ldots,
\end{aligned}
$$

to show how the form of $\mathcal{H}_{0}$ is dictated by imposing (5.35). The simple structure of $\mathcal{H}_{0}$ is at least in part a consequence of the restrictions on counterterms obtained by using dimensional regularisation with minimal subtraction. 


\section{Conclusion}

In this paper we have been primarily concerned with calculational techniques for quantum field theories with a boundary in the simplest case of a scalar field theory. The necessary labour is significantly increased beyond that for field theories without boundary. Even if the manifold on which the quantum field theory is defined is flat, as is usual for applications in statistical physics, the machinery associated with covariant treatments on curved space are a necessary ingredient in our method of calculating divergences for general curved boundaries. In spite of this complexity there are nevertheless potential formal developments associated with the functional Schrödinger equation discussed in the previous section. This may offer a non perturbative approach to discussing quantum field theories, such as in variational methods applied to the effective potential, but it is clearly important to ensure that the functional Hamiltonian operator is well understood in a perturbative context first. A further area of possible relevance of these calculations is to critical phenomena in real systems with boundary, as was the motivation for the work of Diehl and Dietrich [5] whose results for plane boundaries are reproduced here. Even for simple systems there is a rich variety of phase transitions associated with boundaries [4] whose universal properties may be calculated using renormalisable quantum field theories. For the $O(n)$ symmetric model discussed in sections 2 and 3 there is a fixed point for $g=g_{*}, m^{2}=0$ and on the boundary in the Dirichlet case $\hat{\varphi}=0$ while in the Neumann case it is necessary to require $\beta_{c}=0$ or $c=\mathrm{O}(K)$ (for a plane boundary $c=0$ ). More generally if there is a cubic coupling $g_{i j k}$ in $Q(\phi)$ in the treatment of section 3 then from (3.18) to one loop

$$
\hat{\beta}_{i j k}^{\hat{g}}=-\frac{1}{2} \varepsilon \hat{g}_{i j k}+\frac{1}{16 \pi^{2}}\left(-8 \hat{g}_{i \ell m} \hat{g}_{j m n} \hat{g}_{k n \ell}+6 g_{\ell m(i j} \hat{g}_{k) m \ell}-\frac{3}{2} g_{\ell \ell m(i} \hat{g}_{j k) m}\right)
$$

so that there is a possible fixed point for $\hat{g}_{*}=\mathrm{O}\left(\varepsilon^{\frac{1}{2}}\right)$, as discussed by Diehl and Ciach [22] for a single component field.

In connection with the discussion in section 5 it is interesting to note that recently Lüscher et al [23] have considered the Schrödinger wave functional for gauge theories with a view to applying finite size techniques to lattice gauge calculations. Although in other respects more complicated, due to requirements of gauge fixing and introducing ghost fields, the gauge theory case is simpler to the extent that there are no gauge invariant operators with dimensions $<4$ which can occur as boundary counterterms. Lattice calculations of wave functionals are of course well defined non perturbatively but perturbative results, such as obtained here, provide useful constraints on the approach to the continuum limit.

One of us (DMM) would like to thank the Royal Commisssion for the Exhibition of 1851 for an overseas scholarship. 


\section{Appendix A}

The short distance behaviour of the Green function $G_{\Delta}$ near the boundary may be found from an asymptotic expansion of the heat kernel $\mathcal{G}_{\Delta}$ since

$$
G_{\Delta}\left(x, x^{\prime}\right)=\int_{0}^{\infty} d \tau \mathcal{G}_{\Delta}\left(x, x^{\prime} ; \tau\right)
$$

where $\mathcal{G}_{\Delta}$ satisfies

$$
\left(\frac{\partial}{\partial \tau}+\Delta_{x}\right) \mathcal{G}_{\Delta}\left(x, x^{\prime} ; \tau\right)=0, \quad \mathcal{G}_{\Delta}\left(x, x^{\prime} ; 0\right)=\delta^{d}\left(x, x^{\prime}\right)
$$

with boundary conditions corresponding to (1.3)

$$
\left.(1-\mathcal{P}(\hat{\mathbf{x}})) \mathcal{G}_{\Delta}\left(x, x^{\prime} ; \tau\right)\right|_{x=x(\hat{\mathbf{x}})}=0,\left.\quad\left(\mathcal{P}(\hat{\mathbf{x}}) n^{\mu}(\hat{\mathbf{x}}) D_{\mu}+\psi(\hat{\mathbf{x}})\right) \mathcal{G}_{\Delta}\left(x, x^{\prime} ; \tau\right)\right|_{x=x(\hat{\mathbf{x}})}=0
$$

The extension of the DeWitt ansatz for $\mathcal{G}_{\Delta}$ takes the form [13]

$$
\mathcal{G}_{\Delta}\left(x, x^{\prime} ; \tau\right) \simeq \frac{1}{(4 \pi \tau)^{\frac{d}{2}}}\left(e^{-\sigma\left(x, x^{\prime}\right) / 2 \tau} \Omega\left(x, x^{\prime} ; \tau\right)+e^{-\bar{\sigma}\left(x, x^{\prime}\right) / 2 \tau} \bar{\Omega}\left(x, x^{\prime} ; \tau\right)\right)
$$

where $\sigma\left(x, x^{\prime}\right)$ is the geodetic interval based on the geodesic path from $x^{\prime}$ to $x$ while $\bar{\sigma}\left(x, x^{\prime}\right)$ is the corresponding biscalar resulting from the geodesic which undergoes reflection on the boundary. The conventional DeWitt asymptotic expansion [14] is obtained by writing $\Omega\left(x, x^{\prime} ; \tau\right) \simeq \Delta^{\frac{1}{2}}\left(x, x^{\prime}\right) \sum_{n=0} a_{n}\left(x, x^{\prime}\right) \tau^{n}$, with $\Delta^{\frac{1}{2}}\left(x, x^{\prime}\right), a_{n}\left(x, x^{\prime}\right)$ regular for $x \approx x^{\prime}$ and $\Delta^{\frac{1}{2}}(x, x)=a_{0}(x, x)=1$. This expansion determines the singular behaviour of $G_{\Delta}\left(x, x^{\prime}\right)$ for $x^{\prime} \rightarrow x$, as in $(1.5,6)$. The presence of the additional term $\bar{\Omega}$ allows the boundary conditions (A.3) to be satisfied by the full expression in (A.4), while maintaining the usual form for $\Omega$. An appropriate expansion of $\bar{\Omega}$ then enables the singular terms of $G_{\Delta}$ near the boundary to be obtained.

To achieve this in the neighbourhood of the boundary, with coordinates so that the metric takes the form (1.1), both $\sigma$ and $\bar{\sigma}$ are expanded in powers of $\epsilon$, according to (1.9), where to order $\epsilon^{3}$ from I

$$
\begin{aligned}
& \sigma=\frac{1}{2}\left(y-y^{\prime}\right)^{2}+\frac{1}{2} \hat{\gamma}_{i j} \hat{\sigma}^{i} \hat{\sigma}^{j}-\left(y+y^{\prime}\right) \frac{1}{2} K_{i j} \hat{\sigma}^{i} \hat{\sigma}^{j} \\
& \bar{\sigma}=\frac{1}{2}\left(y+y^{\prime}\right)^{2}+\frac{1}{2} \hat{\gamma}_{i j} \hat{\sigma}^{i} \hat{\sigma}^{j}-\frac{y^{2}+y^{\prime 2}}{y+y^{\prime}} \frac{1}{2} K_{i j} \hat{\sigma}^{i} \hat{\sigma}^{j} .
\end{aligned}
$$

Using these results $\bar{\Omega}$ may be obtained as a formal power series in $\epsilon$ with $\bar{\Omega}_{n}=\mathrm{O}\left(\epsilon^{n}\right)$, taking also $\tau=\mathrm{O}\left(\epsilon^{2}\right)$, by solving at each order sets of coupled differential equations in $y$ and $\tau$ with appropriate boundary conditions determined by $\Omega_{n}$ in the analogous expansion of $\Omega$. The solutions of these equations may be expressed in terms of the functions

$$
\begin{aligned}
f_{n}(u, \tau)= & \frac{1}{n !} \frac{1}{(2 \tau)^{n}} \int_{0}^{\infty} d z z^{n} e^{-\frac{z^{2}}{4 \tau}-\frac{z u}{2 \tau}}, \\
& 2 \tau(n+1) f_{n+1}(u, \tau)+u f_{n}(u, \tau)=f_{n-1}(u, \tau)+2 \tau \delta_{n 0}
\end{aligned}
$$


Using (A.5a,b) the results are more conveniently expressed in the form

$$
\mathcal{G}_{\Delta}\left(x, x^{\prime} ; \tau\right) \simeq \frac{1}{(4 \pi \tau)^{\frac{d}{2}}} e^{-\hat{\sigma}\left(\hat{\mathbf{x}}, \hat{\mathbf{x}}^{\prime}\right) / 2 \tau}\left(e^{-v^{2} / 4 \tau} \Omega^{\prime}\left(x, x^{\prime} ; \tau\right)+e^{-u^{2} / 4 \tau} \bar{\Omega}^{\prime}\left(x, x^{\prime} ; \tau\right)\right),
$$

where as before $v=y-y^{\prime}, u=y+y^{\prime}$.

The first two orders in the expansion are straightforward to obtain in terms of the extrinsic curvature of the boundary $K_{i j}(\hat{\mathbf{x}})$ and also $\psi(\hat{\mathbf{x}})$ and the projection operator $\mathcal{P}(\hat{\mathbf{x}})$ appearing in the boundary condition (A.3)

$$
\begin{aligned}
\Omega_{0}^{\prime}= & \hat{I}, \quad \Omega_{1}^{\prime}=u \frac{1}{4} K_{i j} \hat{\sigma}^{i} \hat{\sigma}^{j} \hat{I}, \quad \bar{\Omega}_{0}^{\prime}=\mathcal{P}_{-} \hat{I}, \quad \mathcal{P}_{-}=2 \mathcal{P}-1 \\
\bar{\Omega}_{1}^{\prime}= & \left(K-\frac{1}{\tau} \frac{1}{2} K_{i j} \hat{\sigma}^{i} \hat{\sigma}^{j}\right)\left(\frac{y y^{\prime}}{2 \tau} f_{0}(u, \tau) \mathcal{P}_{-}-2 \tau f_{2}(u, \tau) \mathcal{P}\right) \hat{I} \\
& +\frac{1}{\tau} u \frac{1}{2} K_{i j} \hat{\sigma}^{i} \hat{\sigma}^{j} \mathcal{P}_{-} \hat{I}+f_{0}(u, \tau)(K \mathcal{P}+2 \psi) \hat{I} \\
& -2 \mathcal{P} \hat{\sigma}^{i} \hat{D}_{i} \mathcal{P} \hat{I}+\frac{1}{\tau} f_{0}(u, \tau)\left(-y(1-\mathcal{P}) \hat{D}_{i} \mathcal{P}+y^{\prime} \mathcal{P} \hat{D}_{i} \mathcal{P}\right) \hat{\sigma}^{i} \hat{I} .
\end{aligned}
$$

Using (A.1) and

$$
\int_{0}^{\infty} d \tau \frac{1}{(4 \pi \tau)^{\frac{d}{2}}} \tau^{r+s-1} e^{-\hat{\sigma} / 2 \tau-u^{2} / 4 \tau} f_{s}(u, \tau)=\frac{1}{s !} 2^{2-2 r-s} G_{r, s}(u),
$$

from the definition in (1.12), these results are sufficient to obtain (1.13).

At order $\epsilon^{2}$ the number of terms proliferate and the calculation becomes tedious. Here we consider only those terms which depend on $X$, which represents a mass ${ }^{2}$ term in the operator $\Delta$, and also $\psi$, which has dimensions of mass, appearing in the boundary condition. For the conventional DeWitt expansion the relevant result is very simple

$$
\Omega_{2}^{\prime}=-\tau X^{0} \hat{I},
$$

where $X^{0}=\left.X\right|_{y=0}$. From the results of calculations carried out elsewhere we obtain

$$
\begin{aligned}
\bar{\Omega}_{2}^{\prime}= & \tau\left(X^{0}-2 \mathcal{P} X^{0} \mathcal{P}\right) \hat{I}-\left(y(1-\mathcal{P}) X^{0} \mathcal{P}+y^{\prime} \mathcal{P} X^{0}(1-\mathcal{P})\right) f_{0}(u, \tau) \hat{I} \\
& +\left(\left(y y^{\prime}+2 \tau\right) f_{1}(u, \tau)-10 \tau^{2} f_{3}(u, \tau)\right)\left(2 K-\frac{1}{\tau} K_{i j} \hat{\sigma}^{i} \hat{\sigma}^{j}\right) \psi \hat{I} \\
& +2 \tau f_{1}(u, \tau)\left(K \psi+2 \psi^{2}\right) \hat{I}+K_{i j} \hat{\sigma}^{i} \hat{\sigma}^{j} \psi \hat{I} .
\end{aligned}
$$

The corresponding contributions to the Green function in the expansion (1.11) are

$$
\begin{aligned}
G_{2}^{B}= & -\frac{1}{4} G_{2}^{R}(v) X^{0} \hat{I}, \\
\bar{G}_{2}^{B}= & \frac{1}{4} G_{2}^{R}(u)\left(X^{0}-2 \mathcal{P} X^{0} \mathcal{P}\right) \hat{I}-G_{1,0}(u)\left(y(1-\mathcal{P}) X^{0} \mathcal{P}+y^{\prime} \mathcal{P} X^{0}(1-\mathcal{P})\right) \hat{I} \\
& +G_{1,1}^{R}(u)\left(\frac{4}{3} K \psi+2 \psi^{2}\right) \hat{I}+\left(4 y y^{\prime} G_{0,1}(u)+\frac{5}{3} u G_{0,2}(u)\right) K \psi \hat{I} \\
& -\left(8 y y^{\prime} G_{-1,1}(u)+\frac{2}{3} G_{0,1}(u)+\frac{10}{3} u G_{-1,2}(u)-G_{1}(u)\right) K_{i j} \hat{\sigma}^{i} \hat{\sigma}^{j} \psi \hat{I} .
\end{aligned}
$$

In (A.10)

$$
G_{2}^{R}(u)=G_{2}(u)+\frac{\mu^{-\varepsilon}}{2 \pi^{2} \varepsilon}, \quad G_{1,1}^{R}(u)=G_{1,1}(u)+\frac{\mu^{-\varepsilon}}{4 \pi^{2} \varepsilon},
$$

which ensures that $G_{2}^{R}, G_{1,1}^{R}$ have a smooth limit as $\varepsilon \rightarrow 0$. 


\section{Appendix B}

In this appendix we describe the essential calculational details associated with determining the singular terms arising from the presence of a boundary, as $\varepsilon \rightarrow 0$, of the two loop amplitudes considered in sections 2 and 3. For the graph in fig.1a we need to consider the singular behaviour of the product of two coincident Green functions, i.e. $G|(x) \times G|(x)$, where $G \mid(x)=G(x, x)$. From the results of appendix A the regular part of the coincident limit $\bar{G} \mid(x)$, as given by (1.5), has the general form near the boundary of the region on which $G$ is defined

$$
\begin{aligned}
\bar{G} \mid(x)= & G \mid(x)+\frac{\mu^{-\varepsilon}}{8 \pi^{2} \varepsilon}\left(X(x)-\frac{1}{6} R(x)\right) \\
= & y_{+}^{2-d} e_{d}(\mathbf{x})+y_{+}^{3-d} f_{d}(\mathbf{x})+\frac{2}{\varepsilon}\left(y_{+}^{4-d} g_{d}(\mathbf{x})-\mu^{-\varepsilon} \theta(y) g_{4}(\mathbf{x})\right) \\
& +\frac{2}{\varepsilon}\left(y_{+}^{5-d} h_{d}(\mathbf{x})-\mu^{-\varepsilon} y_{+} h_{4}(\mathbf{x})\right)+H(x)
\end{aligned}
$$

with $H, \partial_{y} H$ regular at $y=0$ for $d \approx 4 . y_{+}^{\lambda-1}$ is a distribution or generalised function with support on $y \geq 0$ and which has a simple pole at $\lambda=-p, p=0,1,2, \ldots[21]$,

$$
y_{+}^{\lambda-1} \sim \frac{1}{\lambda+p} \frac{(-1)^{p}}{p !} \delta^{(p)}(y) .
$$

Furthermore it is important to note that

$$
\frac{1}{\varepsilon}\left(y_{+}^{\alpha \varepsilon-p-1}-y_{+}^{\beta \varepsilon-p-1}\right) \sim \frac{1}{\varepsilon^{2}}\left(\frac{1}{\alpha}-\frac{1}{\beta}\right) \frac{(-1)^{p}}{p !} \delta^{(p)}(y)
$$

with no simple pole in $\varepsilon$, as may be verified by considering Fourier transforms.

Writing for convenience

$$
e_{d}=\frac{\Gamma\left(\frac{1}{2} d-1\right)}{(4 \pi)^{\frac{1}{2} d}}\left(\tilde{e}_{0}+\tilde{e}_{1} \varepsilon+\ldots\right)
$$

as an expansion in $\varepsilon$, and similarly for $f_{d}, g_{d}, h_{d}$, then from (B.2) and (B.3) the singular contributions are, suppressing indices and assuming on the r.h.s. the products are symmetrised so that for instance $\tilde{e}_{0} \times \tilde{f}_{0}=\tilde{f}_{0} \times \tilde{e}_{0}$,

$$
\begin{aligned}
& \bar{G}|\times \bar{G}| \sim \frac{\mu^{-2 \varepsilon}}{\left(16 \pi^{2} \varepsilon\right)^{2}}\{-\varepsilon \tilde{e}_{0} \times \tilde{e}_{0} \frac{1}{12} \delta^{\prime \prime \prime}(y)+\varepsilon \tilde{e}_{0} \times \tilde{f}_{0} \frac{1}{2} \delta^{\prime \prime}(y)-\varepsilon \tilde{f}_{0} \times \tilde{f}_{0} \frac{1}{2} \delta^{\prime}(y) \\
&+2\left(\tilde{e}_{0} \times \tilde{g}_{0}+\varepsilon\left(\tilde{e}_{1} \times \tilde{g}_{0}-\tilde{e}_{0} \times \tilde{g}_{1}\right)\right) \delta^{\prime}(y) \\
&-2\left(\tilde{e}_{0} \times \tilde{h}_{0}+\tilde{f}_{0} \times \tilde{g}_{0}\right. \\
&\left.\left.+\varepsilon\left(\tilde{e}_{1} \times \tilde{h}_{0}-\tilde{e}_{0} \times \tilde{h}_{1}+\tilde{f}_{1} \times \tilde{g}_{0}-\tilde{f}_{0} \times \tilde{g}_{1}\right)\right) \delta(y)\right\} \\
&+\frac{2 \mu^{-\varepsilon}}{16 \pi^{2} \varepsilon}\left(-\tilde{e}_{0} \delta^{\prime}(y)+\tilde{f}_{0} \delta(y)\right) \times H .
\end{aligned}
$$


If we introduce the notation $\left.G\right|^{0}=\left.G\right|_{y \rightarrow 0}$ then from (1.6,7) and (B.1) we may write by analytic continuation in $d$ from $d<2$

$$
\begin{aligned}
\left.G\right|^{0} & =-\frac{2 \mu^{-\varepsilon}}{16 \pi^{2} \varepsilon}\left(X^{0}-\frac{1}{6} R^{0}+\tilde{g}_{0}\right)+H^{0}, \\
\partial_{n}(G \mid)^{0} & =-\frac{2 \mu^{-\varepsilon}}{16 \pi^{2} \varepsilon}\left(\partial_{n} X^{0}-\frac{1}{6} \partial_{n} R^{0}+\tilde{h}_{0}\right)+\partial_{n} H^{0} .
\end{aligned}
$$

In calculations of the divergent parts of amplitudes where $G|\times G|$ appear the non local singular terms present in (B.4) are cancelled by additional counterterms which involve contributions of the form

$$
\begin{aligned}
& -\frac{2 \mu^{-\varepsilon}}{16 \pi^{2} \varepsilon}\left(-\tilde{e}_{0} \delta^{\prime}(y)+\tilde{f}_{0} \delta(y)\right) \times G=-\frac{2 \mu^{-\varepsilon}}{16 \pi^{2} \varepsilon}\left(-\tilde{e}_{0} \delta^{\prime}(y)+\tilde{f}_{0} \delta(y)\right) \times H \\
& \quad-\frac{4 \mu^{-2 \varepsilon}}{\left(16 \pi^{2} \varepsilon\right)^{2}}\left\{\tilde{e}_{0} \times\left(\tilde{g}_{0}+X^{0}\right) \delta^{\prime}(y)-\left(\tilde{e}_{0} \times\left(\tilde{h}_{0}+\partial_{n} X^{0}\right)+\tilde{f}_{0} \times\left(\tilde{g}_{0}+X^{0}\right)\right) \delta(y)\right\} .
\end{aligned}
$$

Using the results from calculations of the asymptotic expansion of the heat kernel we obtain explicitly

$$
\begin{aligned}
\tilde{e}_{0}= & \mathcal{P}_{-}, \quad \tilde{e}_{1}=0, \\
\tilde{f}_{0}= & 4 \psi+\frac{2}{3} K+K \mathcal{P}_{-}, \quad \tilde{f}_{1}=4 \psi+\frac{5}{9} K+\frac{1}{2} K \mathcal{P}_{-} \\
\tilde{g}_{0}= & -X^{0}+2 \mathcal{P} X^{0} \mathcal{P}-4 \psi^{2}-\frac{8}{3} \psi K \\
\tilde{g}_{1}= & -(1-\mathcal{P}) X^{0} \mathcal{P}-\mathcal{P} X^{0}(1-\mathcal{P})-4 \psi^{2}-\frac{11}{9} \psi K \\
\tilde{h}_{0}= & -\partial_{n} X^{0}+(1-\mathcal{P}) \partial_{n} X^{0} \mathcal{P}+\mathcal{P} \partial_{n} X^{0}(1-\mathcal{P})-2 \mathcal{P}_{-} X^{0} \psi-2 \psi X^{0} \mathcal{P}_{-} \\
& +8 \psi^{3}+\frac{16}{3} \psi^{2} K-2 \mathcal{P} X^{0}(1-\mathcal{P}) K-2(1-\mathcal{P}) X^{0} \mathcal{P} K \\
\tilde{h}_{1}= & -\frac{1}{2}\left(\partial_{n} X^{0}-2 \mathcal{P} \partial_{n} X^{0} \mathcal{P}\right)+\frac{1}{2}(1-\mathcal{P}) \partial_{n} X^{0} \mathcal{P}+\frac{1}{2} \mathcal{P} \partial_{n} X^{0}(1-\mathcal{P}) \\
& +2 X^{0} \psi+2 \psi X^{0}-\frac{38}{9} \psi^{2} K \\
& +\frac{1}{2} X^{0} K+\mathcal{P} X^{0} \mathcal{P} K-\frac{13}{3}\left(\mathcal{P} X^{0}(1-\mathcal{P})+\mathcal{P} X^{0}(1-\mathcal{P})\right) K
\end{aligned}
$$

where in $\tilde{g}_{0,1}, \tilde{h}_{0,1}$ we have given only those terms depending on $X, \psi$. For Dirichlet boundary conditions, $\mathcal{P}, \psi=0$ and from (B.5)

$$
\left.G\right|^{0}=H^{0}=0, \quad \partial_{n}(G \mid)^{0}=\partial_{n} H^{0}=0,
$$

so that the last line of (B.4) vanishes in this case. For Neumann boundary conditions $\mathcal{P}=1$ and

$$
\partial_{n}(G \mid)^{0}=-\left.\psi G\right|^{0}-\left.G\right|^{0} \psi, \quad \partial_{n} H^{0}=-\psi H^{0}-H^{0} \psi .
$$

When integrating by parts in integrals over $d v$ it is useful to note that

$$
d v=d S d y\left(1-y K+\mathrm{O}\left(y^{2}\right)\right), \quad \delta^{\prime}(y)=\nabla_{\mu}\left(n^{\mu} \delta(y)\right)+K \delta(y)
$$


with a corresponding formula for $\delta^{\prime \prime}(y)$.

For the amplitude corresponding to the graph in fig.1b it is necessary to determine the singular behaviour of

$$
\begin{aligned}
& \iint d y d y^{\prime} f(y) f\left(y^{\prime}\right) G_{\Delta}\left(x, x^{\prime}\right)^{3} \\
& \quad-\frac{2 \mu^{-\varepsilon}}{16 \pi^{2} \varepsilon} \int d y \frac{\sqrt{\hat{\gamma}}}{\sqrt{g}} f(y)^{2}\left(1^{2} G_{\Delta}(x, x)+1 G_{\Delta}(x, x) 1+G_{\Delta}(x, x) 1^{2}\right) \delta_{\partial \mathcal{M}}\left(\mathbf{x}, \mathbf{x}^{\prime}\right),
\end{aligned}
$$

where $\sqrt{g}=\sqrt{\hat{\gamma}}(1-y K+\ldots)$ and the second term subtracts the subdivergence as usual. $f(y)$ is an arbitrary smooth test function vanishing rapidly as $y \rightarrow \infty$ and $\delta_{\partial \mathcal{M}}\left(\mathbf{x}, \mathbf{x}^{\prime}\right)=$ $\delta^{d-1}\left(\mathbf{x}-\mathbf{x}^{\prime}\right) / \sqrt{\hat{\gamma}(\mathbf{x})}$ denotes the surface $\delta$ function on $\partial \mathcal{M}$. The singular terms as $\varepsilon \rightarrow 0$ arise for $\mathbf{x} \approx \mathbf{x}^{\prime}$ and it is convenient to use directly the representation of $G_{\Delta}$ provided by the heat kernel, as in (A.1), where writing

$$
\frac{1}{(4 \pi \tau)^{\frac{1}{2}(d-1)}} e^{-\hat{\sigma} / 2 \tau \tau \rightarrow 0} \delta_{\partial \mathcal{M}}+\tau\left(\hat{\nabla}^{2}-\frac{1}{3} \hat{R}\right) \delta_{\partial \mathcal{M}}+\ldots
$$

is equivalent to a Taylor expansion of the Fourier transform. For the leading term in the expansion (1.11) it is necessary in (B.11) to take $G_{\Delta} \rightarrow G_{1}(v)+\mathcal{P}_{-} G_{1}(u)$ with $G_{1}(v) \mid=0$. Using (B.12) gives

$G_{1}(v)^{3} \sim \frac{\Gamma\left(\frac{1}{2} d-1\right)^{3}}{64 \pi^{d}} \frac{\pi^{-\frac{1}{2}}}{\Gamma\left(\frac{3}{2} d-3\right)}\left\{\Gamma\left(d-\frac{5}{2}\right)|v|^{-2 d+5} \delta_{\partial \mathcal{M}}+\frac{1}{4} \Gamma\left(d-\frac{7}{2}\right)|v|^{-2 d+7} \hat{\nabla}^{2} \delta_{\partial \mathcal{M}}+\ldots\right\}$,

dropping the $\hat{R}$ term. This is then easily shown, by considering specific test functions such as $f(y)=e^{-\rho y}$, to lead to a simple pole in $\varepsilon$,

$$
\begin{aligned}
& \iint d y d y^{\prime} f(y) f\left(y^{\prime}\right) G_{1}(v)^{3} \\
& \quad \sim \frac{\mu^{-2 \varepsilon}}{2 \varepsilon} \frac{1}{\left(16 \pi^{2}\right)^{2}}\left\{\int_{0}^{\infty} d y f(y)^{2} \hat{\nabla}^{2}-\int_{0}^{\infty} d y f^{\prime}(y)^{2}-f(0) f^{\prime}(0)\right\} \delta_{\partial \mathcal{M}},
\end{aligned}
$$

where the singular contributions arise both from the usual short distance limit $y \rightarrow y^{\prime}$, as for manifolds without boundary, and also $y, y^{\prime} \rightarrow 0$. Similarly for $G_{1}(u)$, although only the latter contribution arises in this case,

$$
\iint d y d y^{\prime} f(y) f\left(y^{\prime}\right) G_{1}(u)^{3} \sim \frac{\mu^{-2 \varepsilon}}{2 \varepsilon} \frac{1}{\left(16 \pi^{2}\right)^{2}} f(0) f^{\prime}(0) \delta_{\partial \mathcal{M}} .
$$

In order to discuss the $G_{1}(v)^{2} G_{1}(u)$ contribution we write, using (B.12) again,

$$
\begin{aligned}
& \left(G_{1}(v)^{2}-\frac{\mu^{-\varepsilon}}{8 \pi^{2} \varepsilon} \delta(v) \delta_{\partial \mathcal{M}}\right) G_{1}(u) \\
& \sim \frac{\Gamma\left(\frac{1}{2} d-1\right)^{2}}{64 \pi^{d}} \lim _{\epsilon \rightarrow 0}\left\{\frac{\pi^{-\frac{1}{2}}}{\Gamma(d-2)} \int_{\epsilon}^{\infty} d t \int_{0}^{\infty} d s \frac{s^{d-3} t^{\frac{1}{2} d-2}}{(s+t)^{\frac{1}{2}(d-1)}} e^{-s v^{2}-t u^{2}}\right. \\
& \left.\quad-\frac{\mu^{-\varepsilon}}{\varepsilon} \delta(v) 2 \pi^{-\frac{1}{2} \varepsilon} \int_{\epsilon}^{\infty} d t t^{\frac{1}{2} d-2} e^{-4 t y^{2}}\right\}
\end{aligned}
$$


where $\epsilon$ plays the role of an infra red regulator so that for $\epsilon>0$ the r.h.s. of (B.16) has a rapid fall off for $y, y^{\prime} \rightarrow \infty$ and we may therefore take $f(y) \rightarrow a+b y$ which allows all integrals to be explicitly evaluated. When $\epsilon \rightarrow 0$ the $\epsilon$ dependence cancels in the terms containing poles in $\varepsilon$, since they arise only from short distance effects, and we find

$$
\iint d y d y^{\prime} f(y) f\left(y^{\prime}\right)\left(G_{1}(v)^{2}-\frac{\mu^{-\varepsilon}}{8 \pi^{2} \varepsilon} \delta(v) \delta_{\partial \mathcal{M}}\right) G_{1}(u) \sim-\frac{\mu^{-2 \varepsilon}}{\left(16 \pi^{2} \varepsilon\right)^{2}}(2-\varepsilon) f(0) f^{\prime}(0) \delta_{\partial \mathcal{M}} .
$$

For the final leading order contribution we obtain

$$
\iint d y d y^{\prime} f(y) f\left(y^{\prime}\right) G_{1}(u)^{2} G_{1}(v) \sim \frac{\mu^{-2 \varepsilon}}{\varepsilon} \frac{1}{\left(16 \pi^{2}\right)^{2}} f(0) f^{\prime}(0) \delta_{\partial \mathcal{M}} .
$$

For pure Neumann, Dirichlet boundary conditions the surface contributions combine to give

$$
\begin{aligned}
\iint d y d y^{\prime} f(y) & f\left(y^{\prime}\right)\left(\left(G_{1}(v) \pm G_{1}(u)\right)^{3} \mp \frac{3 \mu^{-\varepsilon}}{8 \pi^{2} \varepsilon} \delta(v) \delta_{\partial \mathcal{M}} G_{1}(u)\right) \\
& \sim-\frac{6 \mu^{-2 \varepsilon}}{\left(16 \pi^{2} \varepsilon\right)^{2}}\left( \pm 1-\frac{5}{12} \varepsilon \mp \frac{7}{12} \varepsilon\right) f(0) f^{\prime}(0) \delta_{\partial \mathcal{M}}
\end{aligned}
$$

For the next order in the expansion in (1.11) we need to calculate similar results involving $G_{1}^{B}$ and $\bar{G}_{1}^{B}$ as given in (1.13). Although the labour involved increases significantly similar methods may be applied, in particular when there is a subdivergence introducing the regulator $\epsilon$ as in (B.16) so that we can take now $f(y) \rightarrow 1$ and evaluate all integrals before letting $\epsilon \rightarrow 0$.

$$
\begin{aligned}
& \iint d y d y^{\prime} f(y) f\left(y^{\prime}\right) G_{1}(u)^{2} \bar{G}_{1}^{B} \\
& \sim \frac{\mu^{-2 \varepsilon}}{\varepsilon} \frac{1}{\left(16 \pi^{2}\right)^{2}} f(0)^{2}\left(\frac{1}{9} K \mathcal{P}_{-}+\frac{2}{3} K \mathcal{P}+2 \psi\right) \delta_{\partial \mathcal{M}} \\
& \iint d y d y^{\prime} f(y) f\left(y^{\prime}\right) G_{1}(u) G_{1}(v) \bar{G}_{1}^{B} \\
& \sim \frac{\mu^{-2 \varepsilon}}{\varepsilon} \frac{1}{\left(16 \pi^{2}\right)^{2}} f(0)^{2} \frac{1}{3}\left(\frac{5}{8} K \mathcal{P}_{-}+\frac{1}{3} \pi^{2} K \mathcal{P}+\pi^{2} \psi\right) \delta_{\partial \mathcal{M}} \\
& \quad \sim-\frac{\mu^{-2 \varepsilon}}{\left(16 \pi^{2} \varepsilon\right)^{2}} f(0)^{2}\left(\frac{1}{3}\left(1-\frac{11}{12} \varepsilon\right) K \mathcal{P}-+\frac{4}{3}\left(1-\frac{1}{6} \varepsilon\right) K \mathcal{P}+4 \psi\right) \delta_{\partial \mathcal{M}} \\
& \iint d y d y^{\prime} f(y) f\left(y^{\prime}\right)\left(G_{1}(v)^{2}-\frac{\mu^{-\varepsilon}}{8 \pi^{2} \varepsilon} \delta(v) \delta_{\partial \mathcal{M}}\right) \bar{G}_{1}^{B} \\
& \iint d y d y^{\prime} f(y) f\left(y^{\prime}\right) G_{1}(u)^{2} G_{1}^{B} \\
& \sim \frac{\mu^{-2 \varepsilon}}{\varepsilon} \frac{1}{\left(16 \pi^{2}\right)^{2}} f(0)^{2} \frac{1}{4} K \delta_{\partial \mathcal{M}} \\
& \iint d y d y^{\prime} f(y) f\left(y^{\prime}\right) G_{1}(v)^{2} G_{1}^{B}
\end{aligned}
$$




$$
\begin{gathered}
\sim \frac{\mu^{-2 \varepsilon}}{6 \epsilon} \frac{1}{\left(16 \pi^{2}\right)^{2}}\left\{\int_{0}^{\infty} d y y f(y)^{2}\left(K \hat{\nabla}^{2}+2 K^{i j} \hat{\nabla}_{i} \hat{\nabla}_{j}\right)-\int_{0}^{\infty} d y y f^{\prime}(y)^{2} K\right\} \delta_{\partial \mathcal{M}} \\
\iint d y d y^{\prime} f(y) f\left(y^{\prime}\right)\left(G_{1}(v) G_{1}^{B}-\frac{\mu^{-\varepsilon}}{16 \pi^{2} \varepsilon} y K \delta(v) \delta_{\partial \mathcal{M}}\right) G_{1}(u) \\
\sim-\frac{\mu^{-2 \varepsilon}}{\left(16 \pi^{2} \varepsilon\right)^{2}} f(0)^{2} \frac{1}{2}\left(1-\frac{1}{4} \varepsilon\right) K \delta_{\partial \mathcal{M}} .
\end{gathered}
$$

The terms on the r.h.s of (B.20e) correspond to the expansion of $\nabla^{2} \delta^{d}$ around $y, y^{\prime}=0$ to this order in accord with $(1.8), \delta^{d}\left(x, x^{\prime}\right)=(1+y K+\ldots) \delta\left(y-y^{\prime}\right) \delta_{\partial \mathcal{M}}\left(\mathbf{x}, \mathbf{x}^{\prime}\right)$.

For the remaining one particle reducible graph in fig.1c it is necessary because of the form of the counterterms to consider the Neumann and Dirichlet cases separately. The basic Green function may be written as

$$
G_{\Delta}\left(x, x^{\prime}\right)=(1-\mathcal{P}) G^{D}\left(y, y^{\prime}\right)(1-\mathcal{P})+\mathcal{P} G^{N}\left(y, y^{\prime}\right) \mathcal{P}+\ldots
$$

where other contributions are irrelevant in the following discussion and the explicit dependence on $\mathbf{x}, \mathbf{x}^{\prime}$ is suppressed. In the Neumann case it is sufficient to investigate integrals over $y, y^{\prime}$ of the essential form, assuming $\rho_{d}(y)=f(y)\left(e_{d}+y f_{d}\right)$ where $e_{d}(\mathbf{x}), f_{d}(\mathbf{x})$ are given by (B.7) for $\mathcal{P} \rightarrow 1$ to the first order in an expansion in $\varepsilon$ and $f(y)$ is a suitable test function as considered previously,

$$
\begin{aligned}
I^{N}= & \iint d y d y^{\prime} y_{+}^{2-d} y_{+}^{\prime 2-d} \rho_{d}(y) G^{N}\left(y, y^{\prime}\right) \rho_{d}\left(y^{\prime}\right) \\
& -\frac{\mu^{-\varepsilon}}{\varepsilon}\left\{\int d y y_{+}^{2-d} \rho_{d}(y) G^{N}(y, 0)\left(\rho_{4}^{\prime}(0)-\rho_{4}(0) \psi\right)\right. \\
& \left.\quad+\int d y^{\prime} y_{+}^{\prime 2-d}\left(\rho_{4}^{\prime}(0)-\rho_{4}(0) \psi\right) G^{N}\left(0, y^{\prime}\right) \rho_{d}\left(y^{\prime}\right)\right\} \\
& +\frac{\mu^{-2 \varepsilon}}{\varepsilon^{2}}\left(\rho_{4}^{\prime}(0)-\rho_{4}(0) \psi\right) G^{N}(0,0)\left(\rho_{4}^{\prime}(0)-\rho_{4}(0) \psi\right)
\end{aligned}
$$

As remarked in section 3 for the regular part of $G^{N}$, using the appropriate boundary conditions, the counterterms in (B.22) may be integrated by parts to give

$$
I^{N, \text { reg }}=\iint d y d y^{\prime} D(y) G^{N, \text { reg }}\left(y, y^{\prime}\right) D\left(y^{\prime}\right), \quad D(y)=y_{+}^{2-d} \rho_{d}(y)+\frac{\mu^{-\varepsilon}}{\varepsilon} \delta^{\prime}(y) \rho_{4}(y),
$$

where by virtue of (B.2) $D(y)$ is regular, regarded as a distribution on smooth test functions, as $\varepsilon \rightarrow 0$. In order to calculate the poles in $\varepsilon$ arising from this one particle reducible amplitude then it is necessary to only consider the leading singular contributions to $G^{N}$ when $y, y^{\prime} \rightarrow 0$ and also $\mathbf{x}^{\prime} \rightarrow \mathbf{x}$, as exhibited in (1.13) or (1.15).

The most singular term in (1.13) corresponds to taking $G^{N}\left(y, y^{\prime}\right) \rightarrow G_{1}(v)+G_{1}(u)$ and also letting $\psi \rightarrow 0$ in (B.22). Analysing the integrals over $y, y^{\prime}$ in this case gives an 
expansion in powers of $\hat{\sigma}$ of the form

$$
\begin{aligned}
I_{a}^{N}=\alpha & \rho_{d}(0)^{2}(2 \hat{\sigma})^{4-\frac{3}{2} d} \\
& -\frac{1}{\varepsilon} \frac{1}{S_{d-1}}\left(\gamma\left(\rho_{d}(0) \rho_{d}^{\prime}(0)+\rho_{d}^{\prime}(0) \rho_{d}(0)\right)(2 \hat{\sigma})^{\frac{9}{2}-\frac{3}{2} d}\right. \\
& \left.\quad-\delta\left(\rho_{d}(0) \rho_{4}^{\prime}(0)+\rho_{4}^{\prime}(0) \rho_{d}(0)\right)(2 \hat{\sigma})^{\frac{5}{2}-d}\right)+\ldots,
\end{aligned}
$$

for

$$
S_{d}=\frac{2 \pi^{\frac{1}{2} d}}{\Gamma\left(\frac{1}{2} d\right)} .
$$

The remaining terms not shown in (B.24) are less singular as $\hat{\sigma} \rightarrow 0$ and do not lead to poles in $\varepsilon$. The numerical coefficients $\alpha, \gamma$ and $\delta$ may be easily evaluated in terms of gamma functions for general $d$ and as $\varepsilon \rightarrow 0$

$$
\alpha=\mathrm{O}(1), \quad \gamma \sim 1-\varepsilon, \quad \delta \sim \mu^{-\varepsilon}\left(1+\mathrm{O}\left(\varepsilon^{2}\right)\right)
$$

Hence the poles in $\varepsilon$ appear to cancel as expected by virtue of the counterterms present in (B.22). Nevertheless if in the expression obtained in (B.24) $(2 \hat{\sigma})^{-s}$ is regarded as a distribution on $\partial \mathcal{M}$ defined by analytic continuation in $s$ from $s<\frac{1}{2}(d-1)$ then it is non singular when $s \approx 2$, as in the first term of (B.24), but for $s \rightarrow \frac{1}{2}(d-1)$ there is a pole given by

$$
\begin{aligned}
& (2 \hat{\sigma})^{-\frac{1}{2}(d-1)+\frac{1}{2} \lambda} \sim \frac{1}{\lambda} S_{d-1} \delta_{\partial \mathcal{M}} \\
& \frac{1}{\varepsilon}\left((2 \hat{\sigma})^{-\frac{1}{2}(d-1)+\frac{1}{2} a \varepsilon}-(2 \hat{\sigma})^{-\frac{1}{2}(d-1)+\frac{1}{2} b \varepsilon}\right) \sim \frac{1}{\varepsilon^{2}}\left(\frac{1}{a}-\frac{1}{b}\right) S_{d-1} \delta_{\partial \mathcal{M}}+\mathrm{O}(1) \text {. }
\end{aligned}
$$

This result is a direct extension of (B.2) and (B.3). Hence although $I_{a}^{N}$ is a one particle reducible amplitude including counterterms for all sub-divergences it still results in a local divergence represented here by poles in $\varepsilon$ of the form

$I_{a}^{N} \sim \frac{\mu^{-2 \varepsilon}}{\left(16 \pi^{2} \varepsilon\right)^{2}}\left(f(0) f^{\prime}(0)(1+\varepsilon) \tilde{e}_{0} \tilde{e}_{0}+\frac{1}{2} f(0)^{2}\left((1+\varepsilon)\left(\tilde{e}_{0} \tilde{f}_{0}+\tilde{f}_{0} \tilde{e}_{0}\right)-\varepsilon\left(\tilde{e}_{0} \tilde{f}_{1}+\tilde{f}_{1} \tilde{e}_{0}\right)\right)\right) \delta_{\partial \mathcal{M}}$,

where we have inserted the explicit form of $\rho_{d}(y)$, with $f(y)$ an arbitrary smooth test function, and also used $\tilde{e}_{1}=0$ from (B.7). For the terms proportional to $\psi$ then in (B.22) from (1.13) we let $G^{N}\left(y, y^{\prime}\right) \rightarrow 2 \psi G_{1,0}(u)$ or in the $\psi$ dependent counterterms $G^{N}(y, 0) \rightarrow 2 G_{1}(y), G^{N}\left(0, y^{\prime}\right) \rightarrow 2 G_{1}\left(y^{\prime}\right)$. In a similar fashion to the analysis leading to (B.24) and (B.27)

$$
\begin{aligned}
I_{b}^{N} & \sim \frac{1}{\varepsilon} \psi \frac{1}{S_{d-1}}\left(2 \tilde{\gamma} \rho_{d}(0)^{2}(2 \hat{\sigma})^{\frac{9}{2}-\frac{3}{2} d}-\delta\left(\rho_{d}(0) \rho_{4}(0)+\rho_{4}(0) \rho_{d}(0)\right)(2 \hat{\sigma})^{\frac{5}{2}-d}\right)+\ldots \\
& \sim-\frac{\mu^{-2 \varepsilon}}{\left(16 \pi^{2} \varepsilon\right)^{2}} \psi f(0)^{2} \tilde{e}_{0} \tilde{e}_{0} \delta_{\partial \mathcal{M}}
\end{aligned}
$$


where we have used (B.26) again with $\tilde{\gamma}, \mu^{\varepsilon} \delta=1+\mathrm{O}\left(\varepsilon^{2}\right)$. The analogous results for the $K$ dependent terms are obtained in (B.22) by taking

$$
\begin{aligned}
G^{N}\left(y, y^{\prime}\right) \rightarrow & \frac{K}{d-1}\left(2 \hat{\sigma}\left(G_{0}(v)+G_{0}(u)\right) u+2 G_{0}(u) u y y^{\prime}+(d-2) G_{1,0}(u)\right) \\
& +X\left(K-(d-1) \frac{K_{i j} \hat{\sigma}^{i} \hat{\sigma}^{j}}{2 \hat{\sigma}}\right)
\end{aligned}
$$

The various additional terms represented by $X$, which may easily be found from (1.15), may be discarded here as they do not result in any overall divergence since on using

$$
(2 \hat{\sigma})^{-\frac{1}{2}(d+1)+\frac{1}{2} \lambda} \hat{\sigma}_{i} \hat{\sigma}_{j} \sim \frac{1}{\lambda} \frac{S_{d-1}}{d-1} \hat{\gamma}_{i j} \delta_{\partial \mathcal{M}}
$$

in conjunction with (B.26) ensures that the poles in $\varepsilon$ cancel. In this case the counterterms in (B.22) are also irrelevant in obtaining the leading singular term as $\hat{\sigma} \rightarrow 0$ and we obtain

$$
I_{c}^{N}=\frac{K}{d-1} \frac{1}{S_{d-1}} \kappa \rho_{d}(0)^{2}(2 \hat{\sigma})^{\frac{9}{2}-\frac{3}{2} d} \sim \frac{\mu^{-2 \varepsilon}}{\left(16 \pi^{2} \varepsilon\right)^{2}} \frac{1}{2} \varepsilon K f(0)^{2} \tilde{e}_{0} \tilde{e}_{0} \delta_{\partial \mathcal{M}},
$$

since $\kappa=3+\mathrm{O}(\varepsilon)$ (individual contributions to $\kappa$ possess poles in $\varepsilon$ but these cancel when combined together). Combining this with (B.27) and (B.28) gives finally for the singular part of the integral in (B.22) $I^{N}=I_{a}^{N}+I_{b}^{N}+I_{c}^{N}$.

In the corresponding Dirichlet case we are concerned with integrals of the form

$$
\begin{aligned}
I^{D}= & \iint d y d y^{\prime} y_{+}^{2-d} y_{+}^{\prime 2-d} \rho_{d}(y) G^{D}\left(y, y^{\prime}\right) \rho_{d}\left(y^{\prime}\right) \\
& -\frac{\mu^{-\varepsilon}}{\varepsilon}\left\{\left.\int d y y_{+}^{2-d} \rho_{d}(y) G^{D}\left(y, y^{\prime}\right) \overleftarrow{\partial}_{y^{\prime}}\right|_{y^{\prime}=0} \rho_{4}(0)\right. \\
& \left.\quad+\left.\int d y^{\prime} y_{+}^{2-d} \rho_{4}(0) \partial_{y} G^{D}\left(y, y^{\prime}\right)\right|_{y=0} \rho_{d}\left(y^{\prime}\right)\right\} \\
& +\left.\frac{\mu^{-2 \varepsilon}}{\varepsilon^{2}} \rho_{4}(0) \partial_{y} G^{D}\left(y, y^{\prime}\right) \overleftarrow{\partial}_{y^{\prime}}\right|_{y=y^{\prime}=0} \rho_{4}(0)
\end{aligned}
$$

where $\rho_{d}(y)=f(y)\left(e_{d}+y f_{d}\right)$ as before but now $\mathcal{P} \rightarrow 0$. The analysis of the singularities of $I^{D}$ follows similarly to the previous discussion for $I^{N}$. Again for the regular part of $G^{D}$ it is possible to write (B.32) in the analogous form to (B.23), assuming $G^{D, \text { reg }}\left(0, y^{\prime}\right)=$ $G^{D \text {,reg }}(y, 0)=0$, showing that poles in $\varepsilon$ can only arise from the singular short distance part of $G^{D}$ as can be obtained from (1.13) or (1.15). In this case it is necessary to note that $\left.\partial_{y} G^{D} \overleftarrow{\partial}_{y^{\prime}}\right|_{y=y^{\prime}=0}$, which appears in the $1 / \varepsilon^{2}$ counterterm in (B.32), involves terms proportional to $(2 \hat{\sigma})^{-\frac{1}{2} d},(2 \hat{\sigma})^{-\frac{1}{2}(d-1)}$ when $\mathbf{x}^{\prime} \rightarrow \mathbf{x}$, as shown in (C.25), which represent non integrable singularities on integration over $\partial \mathcal{M}$. Furthermore the resulting divergences are not regularised by analytic continuation in $d$. Elsewhere [17] we have discussed how to deal with this problem, which also occurs in the calculation of electrostatic energies on 
$\mathcal{M}$ when the potential is given on $\partial \mathcal{M}$. It is sufficient to treat the singular short distance terms appearing in $\left.\partial_{y} G^{D} \bar{\partial}_{y^{\prime}}\right|_{y=y^{\prime}=0}$ as distributions but it is crucial if correct results are to be obtained to also include an additional term involving $\delta_{\partial \mathcal{M}}$ proportional to $K$ which is shown in (C.25).

For the contributions arising for $G^{D}\left(y, y^{\prime}\right) \rightarrow G_{1}(v)-G_{1}(u)$ then instead of (B.24)

$$
\begin{aligned}
I_{a}^{D}= & \frac{1}{\varepsilon^{2}} \frac{2}{S_{d}}\left(\alpha \rho_{d}(0)^{2}(2 \hat{\sigma})^{4-\frac{3}{2} d}-\beta\left(\rho_{d}(0) \rho_{4}(0)+\rho_{d}(0) \rho_{4}(0)\right)(2 \hat{\sigma})^{2-d}+\mu^{-2 \varepsilon} \rho_{4}(0)^{2}(2 \hat{\sigma})^{-\frac{1}{2} d}\right) \\
+ & \frac{1}{\varepsilon} \frac{1}{S_{d-1}}\left(\gamma\left(\rho_{d}^{\prime}(0) \rho_{d}(0)+\rho_{d}(0) \rho_{d}^{\prime}(0)\right)(2 \hat{\sigma})^{\frac{9}{2}-\frac{3}{2} d}-\delta\left(\rho_{d}^{\prime}(0) \rho_{4}(0)+\rho_{4}(0) \rho_{d}^{\prime}(0)\right)(2 \hat{\sigma})^{\frac{5}{2}-d}\right) \\
& \quad+\ldots,
\end{aligned}
$$

where the numerical coefficients here have the form

$$
\alpha, \gamma \sim 1-\varepsilon, \quad \beta \sim \mu^{-\varepsilon}\left(1-\frac{1}{2} \varepsilon\right), \quad \delta \sim \mu^{-\varepsilon}(1-\varepsilon)
$$

In this case the counterterms contribute to both the leading singular parts shown in (B.33). These are necessary to ensure that only poles in $\varepsilon$ with local residues may appear. Using equations (B.26) and (B.34), the result becomes, with the explicit form of $\rho_{d}(y)$ again,

$I_{a}^{D} \sim-\frac{\mu^{-2 \varepsilon}}{\left(16 \pi^{2} \varepsilon\right)^{2}}\left(f(0) f^{\prime}(0)(1-\varepsilon) \tilde{e}_{0} \tilde{e}_{0}+\frac{1}{2} f(0)^{2}\left((1-\varepsilon)\left(\tilde{e}_{0} \tilde{f}_{0}+\tilde{f}_{0} \tilde{e}_{0}\right)+\varepsilon\left(\tilde{e}_{0} \tilde{f}_{1}+\tilde{f}_{1} \tilde{e}_{0}\right)\right)\right) \delta_{\partial \mathcal{M}}$

For the $K$ dependent terms then using instead of (B.29)

$$
G^{D}\left(y, y^{\prime}\right) \rightarrow \frac{K}{d-1}\left(2 \hat{\sigma}\left(G_{0}(v)-G_{0}(u)\right) u-2 G_{0}(u) u y y^{\prime}\right)+X^{\prime}\left(K-(d-1) \frac{K_{i j} \hat{\sigma}^{i} \hat{\sigma}^{j}}{2 \hat{\sigma}}\right)
$$

yields, since no relevant contributions result from $X^{\prime}$, in the Dirichlet case

$$
\begin{aligned}
I_{b}^{D}= & \frac{1}{\varepsilon} \frac{K}{d-1} \frac{1}{S_{d-1}}\left(2 \kappa \rho_{d}(0)^{2}(2 \hat{\sigma})^{\frac{9}{2}-\frac{3}{2} d}-\lambda\left(\rho_{d}(0) \rho_{4}(0)+\rho_{4}(0) \rho_{d}(0)\right)(2 \hat{\sigma})^{\frac{5}{2}-d}\right) \\
& +\frac{\mu^{-2 \varepsilon}}{\varepsilon^{2}} \frac{d-2}{2(d-1)} K \rho_{4}(0)^{2} \delta_{\partial \mathcal{M}}
\end{aligned}
$$

where the last term is is a consequence of the extra $\delta_{\partial \mathcal{M}}$ contribution shown in (C.25). The coefficients are $\kappa, \mu^{\varepsilon} \lambda \sim 1-2 \varepsilon$ so that (B.36) gives

$$
I_{b}^{D} \sim \frac{\mu^{-2 \varepsilon}}{\left(16 \pi^{2} \varepsilon\right)^{2}} \frac{1}{2} \varepsilon K f(0)^{2} \tilde{e}_{0} \tilde{e}_{0} \delta_{\partial \mathcal{M}} .
$$

The total singular contribution resulting from (B.32) is then $I^{D}=I_{a}^{D}+I_{b}^{D}$ as given by (B.35) and (B.37). 


\section{Appendix C}

For the derivation of the functional Schrödinger equation in section 5 we here summarise the necessary results for variations induced by a deformation of the boundary surface $\partial \mathcal{M}$. For $\partial \mathcal{M}$ parameterised by coordinates $\hat{x}^{i}$ and the embedding in terms of the coordinates for $\mathcal{M}$ specified by $x^{\mu}(\hat{\mathbf{x}})$ we define

$$
e^{\mu}{ }_{i}(\hat{\mathbf{x}})=\frac{\partial x^{\mu}}{\partial \hat{x}^{i}}, \quad n_{\mu} e^{\mu}{ }_{i}=0, \quad n_{\mu} n^{\mu}=1,
$$

with $n^{\mu}(\hat{\mathbf{x}})$ the unit inward normal on $\partial \mathcal{M}$. For a metric $g_{\mu \nu}$ on $\mathcal{M}$ the induced metric on $\partial \mathcal{M}$ is determined by

$$
\hat{\gamma}_{i j}=g_{\mu \nu} e^{\mu}{ }_{i} e_{j}
$$

and then

$$
\begin{aligned}
& \nabla_{i} e^{\mu}{ }_{j}=\partial_{i} e^{\mu}{ }_{j}+\Gamma_{\sigma \rho}^{\mu} e_{i}^{\sigma} e^{\rho}{ }_{j}=e^{\mu}{ }_{k} \hat{\Gamma}_{i j}^{k}+n^{\mu} K_{i j} \\
& \nabla_{i} n^{\mu}=-K_{i j} e^{\mu j}, \quad \partial_{i}=e^{\mu}{ }_{i} \partial_{\mu}
\end{aligned}
$$

defines the extrinsic curvature $K_{i j}=K_{j i}$ of $\partial \mathcal{M}$ for $\Gamma_{\sigma \rho}^{\mu}$ the usual Christoffel connection formed from $g_{\mu \nu}$. If $\hat{\nabla}_{i}$ is defined with the connection $\hat{\Gamma}_{i j}^{k}=\hat{\Gamma}_{j i}^{k}$ acting on tensors on $\partial \mathcal{M}$ then $\hat{\nabla}_{i} \hat{\gamma}_{j k}=0$ so that $\hat{\Gamma}_{i j}^{k}$ is the Christoffel connection formed from $\hat{\gamma}_{j k}$. From (C.3) it is straightforward to derive the Gauss-Codazzi equations relating the Riemann curvature tensor $R_{\mu \nu \sigma \rho}$ for $x \in \partial \mathcal{M}$, with zero and one component along the normal $n^{\mu}$, to the intrinsic Riemann curvature $\hat{R}_{i j k \ell}$ of $\partial \mathcal{M}$ associated with the covariant derivative $\hat{\nabla}_{i}$ and also the extrinsic curvature $K_{i j}$.

For a variation $\delta x^{\mu}=-n^{\mu} \delta t$, as in (5.1), we let

$$
\begin{aligned}
\delta^{\prime} e^{\mu}{ }_{i} & =\partial_{i} \delta x^{\mu}-\delta t n^{\sigma} \Gamma_{\sigma \nu}^{\mu} e^{\nu}{ }_{i} \\
& =\delta t K_{i j} e^{\mu j}-n^{\mu} \partial_{i} \delta t,
\end{aligned}
$$

from (C.3) and hence, regarding $n_{\mu}$ as defined by (C.1),

$$
\delta^{\prime} n_{\mu}=\delta n_{\mu}-n_{\nu} \Gamma_{\sigma \mu}^{\nu} n^{\sigma} \delta t=e_{\mu}^{i} \partial_{i} \delta t .
$$

With the induced metric given by (C.2) we have

$$
\delta \hat{\gamma}_{i j}=2 K_{i j} \delta t
$$

and using $K_{i j}=n_{\mu} \nabla_{i} e^{\mu}$ from (C.3)

$$
\begin{aligned}
\delta K_{i j} & =e_{\nu}{ }^{k} \partial_{k} \delta t \nabla_{i} e^{\nu}{ }_{j}-n_{\mu}\left[\nabla_{n}, \nabla_{i}\right] e^{\mu}{ }_{j}+n_{\mu} \nabla_{i} \delta^{\prime} e^{\mu}{ }_{j} \\
& =-\hat{\nabla}_{i} \partial_{j} \delta t-\left(R_{n j n i}-K_{j k} K_{i}^{k}\right) \delta t .
\end{aligned}
$$

For integrals over a local scalar function $f$ on $\mathcal{M}$ or, restricted to the boundary, on $\partial \mathcal{M}$

$$
\begin{aligned}
\delta \int_{\mathcal{M}} d v f & =\left.\int_{\partial \mathcal{M}} d S \delta t f\right|_{\partial \mathcal{M}} \\
\left.\delta \int_{\partial \mathcal{M}} d S f\right|_{\partial \mathcal{M}} & =\left.\int_{\partial \mathcal{M}} d S \delta t\left(-\partial_{n} f+K f\right)\right|_{\partial \mathcal{M}}
\end{aligned}
$$


neglecting in (C.8b) any dependence of $f$ on $n_{\mu}, \hat{\gamma}_{i j}, K_{i j} \ldots$ where it would be necessary to use results such as (C.4,5,6,7) above.

The deformation of the boundary induces a variation in the Green function $G_{\Delta}$ and the heat kernel $\mathcal{G}_{\Delta}$ since boundary conditions are an essential part of their definitions in (1.4) and (A.2). In the Dirichlet case, with a choice of gauge so that $A_{n}=0$ as in (1.10),

$$
\left.\left(\delta \mathcal{G}_{\Delta}\left(x, x^{\prime} ; \tau\right)-\delta t(\hat{\mathbf{x}}) \partial_{n} \mathcal{G}_{\Delta}\left(x, x^{\prime} ; \tau\right)\right)\right|_{x=x(\hat{\mathbf{x}})}=0
$$

and hence for arbitrary $x, x^{\prime} \in \mathcal{M}$

$$
\delta \mathcal{G}_{\Delta}\left(x, x^{\prime} ; \tau\right)=\left.\int_{0}^{\tau} d \tau^{\prime} \int_{\partial \mathcal{M}} d S \delta t(\hat{\mathbf{x}}) \mathcal{G}_{\Delta}\left(x, \hat{x} ; \tau-\tau^{\prime}\right) \overleftarrow{\partial}_{n} \partial_{n} \mathcal{G}_{\Delta}\left(\hat{x}, x^{\prime} ; \tau^{\prime}\right)\right|_{\hat{x}=x(\hat{\mathbf{x}})}
$$

Analogous results hold for $G_{\Delta}$ as a consequence of (A.1) and therefore

$$
\frac{\delta}{\delta t(\hat{\mathbf{x}})} G_{\Delta}\left(x, x^{\prime}\right)=\left.G_{\Delta}(x, \hat{x}) \overleftarrow{\partial}_{n} \partial_{n} G_{\Delta}\left(\hat{x}, x^{\prime}\right)\right|_{\hat{x}=x(\hat{\mathbf{x}})}
$$

Since by definition $\mathcal{G}_{\Delta}$ is the kernel for the operator $e^{-\tau \Delta}$ it follows from (C.10) that

$$
\delta \operatorname{Tr}\left(e^{-\tau \Delta}\right)=\tau \int_{\partial \mathcal{M}} d S \delta t(\hat{\mathbf{x}}) \operatorname{tr}\left(\left.\partial_{n} \mathcal{G}_{\Delta}\left(x, x^{\prime} ; \tau\right) \overleftarrow{\partial}_{n}^{\prime}\right|_{x=x^{\prime}=x(\hat{\mathbf{x}})}\right)
$$

In general the asymptotic form of the heat kernel is of the form

$$
(4 \pi \tau)^{\frac{1}{2} d} \operatorname{Tr}\left(e^{-\tau \Delta}\right) \sim \int_{\mathcal{M}} d v \sum_{n=0} B_{2 n}(x) \tau^{n}+\int_{\partial \mathcal{M}} d S \sum_{n=1} \hat{B}_{n}(\hat{\mathbf{x}}) \tau^{\frac{1}{2} n}
$$

and we may also write

$$
\left.\tau(4 \pi \tau)^{\frac{1}{2} d} \partial_{n} \mathcal{G}_{\Delta}\left(x, x^{\prime} ; \tau\right) \overleftarrow{\partial_{n}^{\prime}}\right|_{x=x^{\prime}=x(\hat{\mathbf{x}})} \sim \sum_{n=0} \hat{\mathcal{B}}_{n}(\hat{\mathbf{x}}) \tau^{\frac{1}{2} n}
$$

defining $\hat{\mathcal{B}}_{n}(\hat{\mathbf{x}})$ on $\partial \mathcal{M}$. As a consequence of (C.12)

$$
B_{n}+\frac{\delta}{\delta t} \int_{\partial \mathcal{M}} d S \hat{B}_{n}=\operatorname{tr}\left(\hat{\mathcal{B}}_{n}\right)
$$

with $B_{n}=0$ for $n$ odd. This result may be verified using our previous results [13] for $n=0,1,2$,

$$
\hat{\mathcal{B}}_{0}=1, \quad \hat{\mathcal{B}}_{1}=-\frac{1}{2} \sqrt{\pi} K, \quad \hat{\mathcal{B}}_{2}=\frac{1}{6}\left(\hat{R}+K^{2}-K^{i j} K_{i j}\right)-\left.X\right|_{\partial \mathcal{M}}
$$

with $\hat{R}$ the scalar curvature on $\partial \mathcal{M}$. For the discussion in section 5 we need the contribution to $\hat{\mathcal{B}}_{4}$ given by

$$
\hat{\mathcal{B}}_{4}=\left.\left(\frac{1}{2} X^{2}-\frac{1}{2} \nabla_{n}^{2} X-\frac{1}{6} \hat{D}^{2} X+\frac{5}{6} K \partial_{n} X\right)\right|_{\partial \mathcal{M}}+\ldots
$$


where on $\partial \mathcal{M} \nabla_{n}^{2} X=n^{\mu} n^{\nu} \nabla_{\mu} \nabla_{\nu} X$. This has been determined by direct calculation and also checked by using (C.15).

For the functional determinant defined by (1.15) the finite regularised form in four dimensions is given by

$$
\ln \operatorname{det} \Delta_{\mathrm{reg}}=\ln \operatorname{det} \Delta+\frac{2 \mu^{-\varepsilon}}{16 \pi^{2} \varepsilon}\left\{\int_{\mathcal{M}} d v B_{4}+\int_{\partial \mathcal{M}} d S \hat{B}_{4}\right\},
$$

where $B_{4}, \hat{B}_{4}$ are given by (1.16). For general boundary conditions, with $\Delta$ given by (1.2), it is easy to see that, under smooth variations in $X$,

$$
\delta \ln \operatorname{det} \Delta_{\mathrm{reg}}=\int_{\mathcal{M}} d v \operatorname{tr}\left(D_{\Delta} \delta X\right)
$$

where $D_{\Delta}(x)$, as given by (B.1), (B.7) and (B.12), has the form

$$
\begin{aligned}
D_{\Delta}(x)=G_{\Delta}(x, x) & +\frac{2 \mu^{-\varepsilon}}{16 \pi^{2} \varepsilon}\left(X(x)-\frac{1}{6} R(x)\right) \\
& +\frac{\mu^{-\varepsilon}}{16 \pi^{2} \varepsilon}\left(\nabla_{\mu}\left(n^{\mu}(\hat{\mathbf{x}}) \mathcal{P}_{-}(\hat{\mathbf{x}}) \delta_{n}(x)\right)-\left(4 \psi(\hat{\mathbf{x}})+\frac{2}{3} K(\hat{\mathbf{x}})\right) \delta_{n}(x)\right)
\end{aligned}
$$

with $\delta_{n}(x)$ the surface $\delta$ function defined by $d v(x) \delta_{n}(x)=d S(\hat{\mathbf{x}})$, in the standard coordinates of $(1.1) \delta_{n}(x)=\delta(y), \nabla_{\mu}\left(n^{\mu}(\hat{\mathbf{x}}) \delta_{n}(x)\right)=\delta^{\prime}(y)-K(\hat{\mathbf{x}}) \delta(y)$. This represents the regularised form of $G_{\Delta}(x, x)$ in dimensional regularisation with both short distance and boundary divergences removed by subtraction of poles in $\varepsilon$. In the Dirichlet case, when $\mathcal{P}_{-} \rightarrow 1$, we may also find for variations of the boundary, from (C.12) and (C.15),

$$
\frac{\delta}{\delta t(\hat{\mathbf{x}})} \ln \operatorname{det} \Delta_{\text {reg }}=-\operatorname{tr}\left(\mathcal{K}_{\Delta}(\hat{\mathbf{x}}, \hat{\mathbf{x}})\right)+\frac{2 \mu^{-\varepsilon}}{16 \pi^{2} \varepsilon} \operatorname{tr}\left(\hat{\mathcal{B}}_{4}(\hat{\mathbf{x}})\right)
$$

where formally

$$
\mathcal{K}_{\Delta}\left(\hat{\mathbf{x}}, \hat{\mathbf{x}}^{\prime}\right)=\left.\partial_{n} G_{\Delta}\left(x, x^{\prime}\right) \overleftarrow{\partial}_{n}^{\prime}\right|_{x=x(\hat{\mathbf{x}}), x^{\prime}=x\left(\hat{\mathbf{x}}^{\prime}\right)}
$$

For applications in section 5 it is necessary to also consider variations of the Green function when arguments lie on the boundary. By careful analysis, assuming $\hat{f}(\hat{\mathbf{x}})$ and $f(x)$ are smooth test functions on $\partial \mathcal{M}$ and $\mathcal{M}$,

$$
\begin{aligned}
& \frac{\delta}{\delta t(\hat{\mathbf{x}})} \int d S^{\prime} d v^{\prime \prime}\left.\hat{f}\left(\hat{\mathbf{x}}^{\prime}\right) \partial_{n}^{\prime} G_{\Delta}\left(x^{\prime}, x^{\prime \prime}\right)\right|_{x^{\prime}=x\left(\hat{\mathbf{x}}^{\prime}\right)} f\left(x^{\prime \prime}\right) \\
&=\left.\int d S^{\prime} d v^{\prime \prime} \hat{f}\left(\hat{\mathbf{x}}^{\prime}\right) \mathcal{K}_{\Delta}\left(\hat{\mathbf{x}}^{\prime}, \hat{\mathbf{x}}\right) \partial_{n} G_{\Delta}\left(x, x^{\prime \prime}\right)\right|_{x=x(\hat{\mathbf{x}})} f\left(x^{\prime \prime}\right) \\
& \quad+\hat{f}(\hat{\mathbf{x}}) f(x(\hat{\mathbf{x}}))
\end{aligned}
$$


Also, in a similar fashion,

$$
\begin{aligned}
& \frac{\delta}{\delta t(\hat{\mathbf{x}})} \int d S^{\prime} d S^{\prime \prime} \hat{f}\left(\hat{\mathbf{x}}^{\prime}\right) \mathcal{K}_{\Delta}\left(\hat{\mathbf{x}}^{\prime}, \hat{\mathbf{x}}^{\prime \prime}\right) \hat{f}\left(\hat{\mathbf{x}}^{\prime \prime}\right) \\
&=\int d S^{\prime} d S^{\prime \prime} \hat{f}\left(\hat{\mathbf{x}}^{\prime}\right) \mathcal{K}_{\Delta}\left(\hat{\mathbf{x}}^{\prime}, \hat{\mathbf{x}}\right) \mathcal{K}_{\Delta}\left(\hat{\mathbf{x}}, \hat{\mathbf{x}}^{\prime \prime}\right) \hat{f}\left(\hat{\mathbf{x}}^{\prime \prime}\right) \\
& \quad-\hat{\gamma}^{i j}(\hat{\mathbf{x}}) \partial_{i} \hat{f}(\hat{\mathbf{x}}) \partial_{j} \hat{f}(\hat{\mathbf{x}})-\hat{f}(\hat{\mathbf{x}}) X(x(\hat{\mathbf{x}})) \hat{f}(\hat{\mathbf{x}}),
\end{aligned}
$$

which is analogous to a result quoted by Symanzik*.

In general the coefficients $\hat{\mathcal{B}}_{n}$ are related to the short distance expansion of $\mathcal{K}_{\Delta}\left(\hat{\mathbf{x}}, \hat{\mathbf{x}}^{\prime}\right)$ when $\hat{\mathbf{x}}^{\prime} \approx \hat{\mathbf{x}}$. We obtain

$$
\begin{aligned}
\mathcal{K}_{\Delta} \sim & \frac{\Gamma\left(\frac{1}{2} d\right)}{\pi^{\frac{1}{2} d}}(2 \hat{\sigma})^{-\frac{1}{2} d} \hat{I}+\frac{d-2}{2(d-1)} K \delta_{\partial \mathcal{M}} \\
& -\frac{\Gamma\left(\frac{1}{2} d-\frac{1}{2}\right)}{4 \pi^{\frac{1}{2} d-\frac{1}{2}}}(2 \hat{\sigma})^{\frac{1}{2}-\frac{1}{2} d}\left(K-(d-1) \frac{K_{i j} \hat{\sigma}^{i} \hat{\sigma}^{j}}{2 \hat{\sigma}}\right) \hat{I} .
\end{aligned}
$$

The term proportional to $\delta_{\partial \mathcal{M}}$ results from a careful treatment of the singular behaviour of $\mathcal{K}$ at coincident points [17]. The result given is compatible with treating $(2 \hat{\sigma})^{-s}$ as a distribution [21] or in integrating the kernel $\mathcal{K}\left(\hat{\mathbf{x}}, \hat{\mathbf{x}}^{\prime}\right)$ with a smooth function $\hat{f}\left(\hat{\mathbf{x}}^{\prime}\right)$ excluding a disc of radius $c$ centred on $\hat{\mathbf{x}}$ and subtracting a term $\propto c^{-1}$ to ensure a finite result as $c \rightarrow 0$.

* See eq. (6.6c) in ref. [10] 


\section{References}

[1] H.B.G. Casimir, Proc. Kon. Nederl. Akad. Wet. B51 (1948) 793;

H.B.G. Casimir and D. Polder, Phys. Rev. 73 (1948) 360.

[2] G. Plumien, B. Muller and W. Greiner, Phys. Reports 134 (1986) 87.

[3] S. Blau, M. Visser and A. Wipf, Nucl. Phys. B310 (1988) 163;

E. Elizalde and A. Romeo, Int. J. Mod. Phys. A5 (1990) 1653; J. Math. Phys. 30 (1989) 1133, (E) 31 (1990) 31.

[4] H.W. Diehl, 'Phase Transitions and Critical Phenomena', vol 10, p. 75, (C. Domb and J.L. Lebowitz eds.) Academic Press, London (1986).

[5] H.W. Diehl, and S. Dietrich, Zeit. f. Phys. B 42 (1981) 65, (E) 43 (1981) 281; 50 (1983) 117.

[6] A. Chodos, R.L. Jaffe, K. Johnson, C.B. Thorn and V.F. Weisskopf, Phys. Rev. D9 (1974) 3471;

A. Chodos and C.B. Thorn, Phys. Lett. 53B (1974) 359;

T.H. Hansson and R.L. Jaffe, Phys. Rev. D28 (1983) 882;

S.N. Goldhaber, T.H. Hansson and R. L. Jaffe, Phys. Lett. 131B (1983) 445; Nucl. Phys. B277 (1986) 674;

J. Baacke and Y. Igarashi, Phys. Rev. D27 (1983) 460.

[7] R. Balian and C. Bloch, Ann. Phys. (N.Y.) 60 (1970) 401; 64 (1972) 271; 69 (1974) 76; (E) (1974) 84 554;

R. Balian and B. Duplantier, Ann. Phys. (N.Y.) 104 (1977) 300; 112 (1978) 105;

T.H. Hansson and R.L. Jaffe, Ann. Phys. (N.Y.) 151 (1983) 204;

R.L. Jaffe and L.C. Vintro, Ann. Phys. (N.Y.) 162 (1985) 212.

[8] O. Alvarez, Nucl. Phys. B216 (1983) 125;

H. Dorn and H-J. Otto, Zeit. f. Phys. C 32 (1986) 599;

A. Abouelsaood, C.G. Callan, C.R. Nappi and S.A. Yost, Nucl. Phys. B280 [FS18] (1987) 599;

C.G. Callan, C. Lovelace, C.R. Nappi, and S.A. Yost, Nucl. Phys. B288 (1987) 525 ;

A.A. Tseytlin, Nucl. Phys. B276 (1986) 391; 
O.D. Andreev and A.A. Tseytlin, Mod. Phys. Lett. 3 (1988) 1349;

C.G. Callan and L. Thorlacius, Nucl. Phys. B319 (1989) 133; B329 (1990) 117;

H. Luckock, Ann. Phys. (N.Y.) 194 (1989) 113;

Z. Jaskólski, Comm. Math. Phys. 128 (1990) 285.

[9] J.L. Cardy, Nucl. Phys. B240 [FS12] (1984) 514;

J.L. Cardy, Nucl. Phys. B275 [FS17] (1986) 200;

J.L. Cardy, Nucl. Phys. B324 (1989) 581;

J.L. Cardy, Phys. Rev. Lett. 65 (1990) 1443;

J.L. Cardy and D.C. Lewellen, Phys. Lett. 259B (1991) 274;

D.C. Lewellen, Nucl. Phys. B372 (1992) 654.

[10] K. Symanzik, Nucl. Phys. B190 [FS3] (1981) 1;

M. Lüscher, Nucl. Phys. B254 (1985) 52.

[11] M. Lüscher, K. Symanzik and P. Weisz, Nucl. Phys. B173 (1980) 365.

[12] J.B. Hartle and S.W. Hawking, Phys. Rev. D28 (1983) 2960;

J.L. Halliwell and J.B. Hartle, Phys. Rev. D43 (1991) 1170.

[13] D.M. McAvity and H. Osborn, Classical and Quantum Gravity, 8 (1991) 603; 1445; (E) 9 (1991) 317;

D.M. McAvity, Classical and Quantum Gravity, in press, Cambridge preprint, DAMTP 92-17.

[14] B.S. DeWitt, 'Dynamical Theory of Groups and Fields', Gordon and Breach, London (1965).

[15] D.I. Jack and H. Osborn, Nucl. Phys. B324 (1984) 331.

[16] I.G. Moss and J.S. Dowker, Phys. Lett. 229B (1989) 261;

J.S Dowker and J.P. Schofield, J. Math. Phys. 31 (1990) 808;

T.P. Branson and P.B. Gilkey, Comm. Partial Diff. Eqs. 15 (1990) 245;

A. Dettki and A. Wipf, preprint ETH-TH/91-19.

[17] D.M. McAvity and H. Osborn, Journal of Physics A, 25 (1992) 3287.

[18] D.I. Jack and H. Osborn, Nucl. Phys. B343 (1990) 647. 
H. Osborn, Nucl. Phys. B363 (1991) 486.

[19] J. Polchinski, Nucl. Phys. B303 (1988) 226.

[20] L.S. Schulman, 'Techniques and Applications of Path Integrals', John Wiley and Sons, New York (1981).

[21] I.M. Gel'Fand and G.E. Shilov, 'Generalised Functions', volume 1, (Academic Press, 1964).

[22] H.W. Diehl and A. Ciach, Phys. Rev. B44 (1991) 6642.

[23] M. Lüscher, R. Narayanan, P. Weisz and U. Wolff, Desy preprint, DESY 92-025. 